\title{
COGRADE CONDITIONS AND COTORSION PAIRS
}

\author{
XI TANG AND ZHAOYONG HUANG
}

\begin{abstract}
Let $R$ and $S$ be rings and ${ }_{R} \omega_{S}$ a semidualizing bimodule. We study when the double functor $\operatorname{Tor}_{i}^{S}\left(\omega, \operatorname{Ext}_{R}^{i}(\omega,-)\right)$ preserves epimorphisms and the double functor $\operatorname{Ext}_{R}^{i}\left(\omega, \operatorname{Tor}_{i}^{S}(\omega,-)\right)$ preserves monomorphisms in terms of the (strong) cograde conditions of modules. Under certain cograde condition of modules, we construct two complete cotorsion pairs. In addition, we establish the relation between some relative finitistic dimensions of rings and the right and left projective dimensions of $\omega$.
\end{abstract}

\section{Introduction}

Let $R$ be a left and right Noetherian ring and $n \geqslant 1$. It was proved by Auslander that the flat dimension of the $i$-th term in the minimal injective resolution of $R_{R}$ is at most $i$ for any $0 \leqslant i<n$ if and only if the strong grade of $\operatorname{Ext}_{R}^{i}(M, R)$ is at least $i$ for any finitely generated left $R$-module $M$ and $1 \leqslant i \leqslant n$; and this result is left-right symmetric ([16, Theorem 3.7]). In this case, $R$ is called Auslander $n$-Gorenstein. If $R$ is Auslander $n$-Gorenstein for all $n$, then it is said to satisfy the Auslander condition. This condition is a non-commutative version of commutative Gorenstein rings. It has been known that Auslander $n$-Gorenstein rings and the Auslander condition play a crucial role in homological algebra, representation theory of artin algebras and non-commutative algebraic geometry, see [5, 6, 10, 12, 15, 16, 20, 22, 24, 25, 26, 27, 28 and references therein. In particular, Auslander $n$-Gorenstein rings and some generalized versions were characterized in terms of the properties of the double functor $\operatorname{Ext}_{R^{o p}}^{i}\left(\operatorname{Ext}_{R}^{i}(-, R), R\right)$ and certain (strong) grade conditions of Ext-modules, and a series of cotorsion pairs were constructed under the Auslander condition ([24]).

It is well known that the (Auslander) transpose is one of the most powerful tools in representation theory of artin algebras and Gorenstein homological algebra, see [4, 7. 14. To dualize this important and useful notion, we introduced in 32] the notion of the cotranspose of modules and then obtained many dual counterparts of interesting results $(32,33,34,35)$. As a dual of the notion of the (strong) grade of modules, we introduced in [32, 33. the notion of the (strong) cograde of modules, and obtained the dual versions of some results about the (strong) grade of modules. Let $R$ and $S$ be rings and ${ }_{R} \omega_{S}$ a semidualizing bimodule. In this paper, we will study when the double functor $\operatorname{Tor}_{i}^{S}\left(\omega, \operatorname{Ext}_{R}^{i}(\omega,-)\right)$ preserves epimorphisms and the double functor $\operatorname{Ext}_{R}^{i}\left(\omega, \operatorname{Tor}_{i}^{S}(\omega,-)\right)$ preserves monomorphisms in terms of the (strong) cograde conditions of modules and some related properties of the cotranspose of modules, and also investigate the relationship between certain cograde

2010 Mathematics Subject Classification. 18G25, 16E10, 16E30.

Key words and phrases: Semidualizing bimodules; (Strong) Ext-cograde, (Strong) Tor-cograde; Double functors; $n$ - $\mathcal{X}$-(co)syzygy; (Adjoint) $n$-cotorsionfree modules; Cotorsion pairs; Finitistic dimensions. 
conditions of modules and complete cotorsion pairs. This paper is organized as follows.

In Section 2, we give some terminology and some preliminary results.

Let $R$ and $S$ be rings and ${ }_{R} \omega_{S}$ a semidualizing bimodule. In Section 3, we study when $\operatorname{Tor}_{i}^{S}\left(\omega, \operatorname{Ext}_{R}^{i}(\omega,-)\right)$ preserves epimorphisms and $\operatorname{Ext}_{R}^{i}\left(\omega, \operatorname{Tor}_{i}^{S}(\omega,-)\right)$ preserves monomorphisms in terms of the (strong) cograde conditions of modules. Let $n, k \geqslant 0$. We prove that the Tor-cograde of $\operatorname{Ext}_{R}^{i+k}(\omega, M)$ with respect to $\omega$ is at least $i$ for any left $R$-module $M$ and $1 \leqslant i \leqslant n$ if and only if $\operatorname{Tor}_{i}^{S}\left(\omega, \operatorname{Ext}_{R}^{i}(\omega, f)\right)$ is an epimorphism for any epimorphism of left $R$-modules $f: B \rightarrow C$ with $B, C$ being a $(k+1)$-cosyzygy and $0 \leqslant i \leqslant n-1$ (Theorem 3.5); and that the Ext-cograde of $\operatorname{Tor}_{i+k}^{S}(\omega, N)$ with respect to $\omega$ is at least $i$ for any left $S$-module $N$ and $1 \leqslant i \leqslant n$ if and only if $\operatorname{Ext}_{R}^{i}\left(\omega, \operatorname{Tor}_{i}^{S}(\omega, g)\right)$ is a monomorphism for any monomorphism of left $S$-modules $g: B^{\prime} \longmapsto C^{\prime}$ with $B^{\prime}, C^{\prime}$ being a $(k+1)$-yoke and $0 \leqslant i \leqslant n-1$ (Theorem 3.77).

Moreover, we prove that the strong Tor-cograde of $\operatorname{Ext}_{R}^{i+k}(\omega, M)$ with respect to $\omega$ is at least $i$ for any left $R$-module $M$ and $1 \leqslant i \leqslant n$ if and only if for any exact sequence of left $R$-modules $0 \rightarrow A \rightarrow B \stackrel{f}{\longrightarrow} C \rightarrow 0$ with $A$ an $(i-1)-\mathcal{P}_{\omega}(R)$ syzygy of an $(i+k-1)$-cosyzygy, $\operatorname{Tor}_{i}^{S}\left(\omega, \operatorname{Ext}_{R}^{i}(\omega, f)\right)$ is an epimorphism for any $0 \leqslant i \leqslant n-1$ (Theorem 3.8); and that the strong Ext-cograde of $\operatorname{Tor}_{i+k}^{S}(\omega, N)$ with respect to $\omega$ is at least $i$ for any left $S$-module $N$ and $1 \leqslant i \leqslant n$ if and only if for any exact sequence of left $S$-modules $0 \rightarrow A \stackrel{g}{\rightarrow} B \rightarrow C \rightarrow 0$ with $C$ an $(i-1)$ $\mathcal{I}_{\omega}(S)$-cosyzygy of an $(i+k-1)$-yoke, $\operatorname{Ext}_{R}^{i}\left(\omega, \operatorname{Tor}_{i}^{S}(\omega, g)\right)$ is a monomorphism for any $0 \leqslant i \leqslant n-1$ (Theorem 3.9).

In Section 4, we introduce the notion of $\omega$ satisfying the (quasi) $n$-cograde condition in terms of the properties of the strong cograde of modules. By using the results obtained in Section 3, we give some equivalent characterizations for $\omega$ satisfying such conditions (Theorems 4.8 and 4.14). In particular, the $n$-cograde condition is left-right symmetric, but the quasi $n$-cograde condition is not. In addition, we prove that the Tor-cograde of $\operatorname{Ext}_{R}^{i}(\omega, M)$ with respect to $\omega$ is at least $i-1$ for any $M \in \operatorname{Mod} R$ and $1 \leqslant i \leqslant n$ if and only if the Ext-cograde of $\operatorname{Tor}_{i}^{S}(\omega, N)$ with respect to $\omega$ is at least $i-1$ for any $N \in \operatorname{Mod} S$ and $1 \leqslant i \leqslant n$ (Theorem 4.19).

In Section 5, we prove that if one of the equivalent conditions in Theorem 4.19 mentioned above is satisfied, then the right $S$-projective dimension $\operatorname{pd}_{S^{\circ}} \omega$ of $\omega$ is at most $n-1$ if and only if $\left(\mathcal{P}_{\omega}\right.$ - id $\left.{ }^{\leqslant n-1}(R),{ }_{R} \omega^{\perp_{n}}\right)$ forms a complete cotorsion pair; and the left $R$-projective dimension $\operatorname{pd}_{R} \omega$ of $\omega$ is at most $n-1$ if and only if $\left(\omega_{S}{ }^{T_{n}}, \mathcal{I}_{\omega}\right.$ - $\left.\mathrm{pd}^{\leqslant n-1}(S)\right)$ forms a complete cotorsion pair (Theorem 5.6); see Section 2 and 5 for the details of these notations. Then we apply this result to right quasi $(n-1)$-Gorenstein artin algebras (Corollary 5.8$)$.

In Section 6, we introduce the finitistic $\mathcal{P}_{\omega}(R)$-injective dimension $\mathrm{FP}_{\omega}$-id $R$ of $R$ and the $\mathcal{I}_{\omega}(S)$-projective dimension $\mathrm{FI}_{\omega}$-pd $S$ of $S$. We prove that if the Tor-cograde of $\operatorname{Ext}_{R}^{i+k}(\omega, M)$ with respect to $\omega$ is at least $i$ for any $M \in \operatorname{Mod} R$ and $i \geqslant 1$, then $\mathrm{FP}_{\omega}$-id $R \leqslant \operatorname{pd}_{R} \omega \leqslant \mathrm{FP}_{\omega}$-id $R+k$; and if the Ext-cograde of $\operatorname{Tor}_{i+k}^{S}(\omega, N)$ with respect to $\omega$ is at least $i$ for any $N \in \operatorname{Mod} S$ and $i \geqslant 1$, then $\mathrm{FI}_{\omega}-\mathrm{pd} S \leqslant \operatorname{pd}_{S^{o p}} \omega \leqslant \mathrm{F} \mathcal{I}_{\omega}-\operatorname{pd} S+k$ (Theorem 6.3). As an application, we get that for an artin algebra $R$, if $R$ satisfies the Auslander condition, then FPD $R^{o p}=$ FID $R^{o p}=\operatorname{id}_{R^{o p}} R=\operatorname{id}_{R} R=\mathrm{FPD} R=$ FID $R$; and if $R$ satisfies the right quasi Auslander condition, then FPD $R \leqslant$ FID $R=\operatorname{id}_{R^{o p}} R=\operatorname{id}_{R} R \leqslant \operatorname{FPD} R+1$, where 
FID $R$, FPD $R, \operatorname{id}_{R^{o p}} R$ and $\operatorname{id}_{R} R$ are the finitistic injective dimension, the finitistic projective dimension, the right and left self-injective dimensions of $R$ respectively (Corollary 6.9).

\section{Preliminaries}

Throughout this paper, all rings are associative rings with units. For a ring $R$, $\operatorname{Mod} R(\operatorname{resp} . \bmod R)$ are the class of left (resp. finitely generated left) $R$-modules. Let $M \in \operatorname{Mod} R$, we use $\operatorname{Add}_{R} M$ to denote the subclass of $\operatorname{Mod} R$ consisting of modules consisting of direct summands of direct sums of copies of $M$, and use $\operatorname{pd}_{R} M, \mathrm{fd}_{R} M$ and $\operatorname{id}_{R} M$ to denote the projective, flat and injective dimensions of $M$ respectively.

Definition 2.1. ([2, 19]). Let $R$ and $S$ be rings. An $(R-S)$-bimodule ${ }_{R} \omega_{S}$ is called semidualizing if the following conditions are satisfied.

(a1) ${ }_{R} \omega$ admits a degreewise finite $R$-projective resolution.

(a2) $\omega_{S}$ admits a degreewise finite $S$-projective resolution.

(b1) The homothety $\operatorname{map}_{R} R_{R} \stackrel{R \gamma}{\rightarrow} \operatorname{Hom}_{S^{\circ o p}}(\omega, \omega)$ is an isomorphism.

(b2) The homothety map ${ }_{S} S_{S} \stackrel{\gamma_{S}}{\rightarrow} \operatorname{Hom}_{R}(\omega, \omega)$ is an isomorphism.

(c1) $\operatorname{Ext}_{R} \geqslant 1(\omega, \omega)=0$.

(c2) $\operatorname{Ext}_{S^{o p}} \stackrel{2}{2}(\omega, \omega)=0$.

Wakamatsu in 37] introduced and studied the so-called generalized tilting modules, which are usually called Wakamatsu tilting modules, see [8, 29]. Note that a bimodule ${ }_{R} \omega_{S}$ is semidualizing if and only if it is Wakamatsu tilting (39, Corollary 3.2]). Examples of semidualizing bimodules are referred to [19, 31, 33, 35, 38.

From now on, $R$ and $S$ are arbitrary rings and we fix a semidualizing bimodule ${ }_{R} \omega_{S}$. For convenience, We write

$$
\begin{aligned}
(-)_{*} & :=\operatorname{Hom}(\omega,-) \text { and }(-)^{*}:=\operatorname{Hom}(-, \omega), \\
R^{\omega^{\perp}}: & =\left\{M \in \operatorname{Mod} R \mid \operatorname{Ext}_{R}^{\geqslant 1}(\omega, M)=0\right\}, \\
\omega_{S}{ }^{\top} & :=\left\{N \in \operatorname{Mod} S \mid \operatorname{Tor}_{\geqslant 1}^{S}(\omega, N)=0\right\} .
\end{aligned}
$$

For any $n \geqslant 1$, we write

$$
\begin{aligned}
R \omega^{\perp_{n}} & :=\left\{M \in \operatorname{Mod} R \mid \operatorname{Ext}_{R}^{1 \leqslant i \leqslant n}(\omega, M)=0\right\}, \\
\omega_{S}{ }^{\top}{ }_{n} & :=\left\{N \in \operatorname{Mod} S \mid \operatorname{Tor}_{1 \leqslant i \leqslant n}^{S}(\omega, N)=0\right\} ;
\end{aligned}
$$

in particular, $R^{\omega^{\perp_{0}}}=\operatorname{Mod} R$ and $\omega_{S}{ }^{\top_{0}}=\operatorname{Mod} S$. Symmetrically, $\omega_{S}^{\perp_{n}}$ and $R_{R} \omega^{\top_{n}}$ are defined. Following [19, set

$$
\begin{gathered}
\mathcal{F}_{\omega}(R):=\left\{\omega \otimes_{S} F \mid F \text { is flat in } \operatorname{Mod} S\right\}, \\
\mathcal{P}_{\omega}(R):=\left\{\omega \otimes_{S} P \mid P \text { is projective in } \operatorname{Mod} S\right\}, \\
\mathcal{I}_{\omega}(S):=\left\{I_{*} \mid I \text { is injective in } \operatorname{Mod} R\right\} .
\end{gathered}
$$

The modules in $\mathcal{F}_{\omega}(R), \mathcal{P}_{\omega}(R)$ and $\mathcal{I}_{\omega}(S)$ are called $\omega$-flat, $\omega$-projective and $\omega$ injective respectively. Note that $\mathcal{P}_{\omega}(R)=\operatorname{Add}_{R} \omega$ ([33, Proposition 3.4(2)]). Symmetrically, the classes of $\mathcal{F}_{\omega}\left(S^{o p}\right), \mathcal{P}_{\omega}\left(S^{o p}\right)$ and $\mathcal{I}_{\omega}\left(R^{o p}\right)$ are defined.

Let $M \in \operatorname{Mod} R$ and $N \in \operatorname{Mod} S$. Then we have the following two canonical valuation homomorphisms

$$
\theta_{M}: \omega \otimes_{S} M_{*} \rightarrow M
$$


defined by $\theta_{M}(x \otimes f)=f(x)$ for any $x \in \omega$ and $f \in M_{*}$; and

$$
\mu_{N}: N \rightarrow\left(\omega \otimes_{S} N\right)_{*}
$$

defined by $\mu_{N}(y)(x)=x \otimes y$ for any $y \in N$ and $x \in \omega$. Recall that a module $M \in \operatorname{Mod} R$ is called $\omega$-cotorsionless (resp. $\omega$-coreflexive) if $\theta_{M}$ is an epimorphism (resp. an isomorphism) (32]); and a module $N \in \operatorname{Mod} S$ is called adjoint $\omega$ cotorsionless (resp. adjoint $\omega$-coreflexive) if $\mu_{N}$ is a monomorphism (resp. an isomorphism) ([34).

Definition 2.2. ([19]).

(1) The Auslander class $\mathcal{A}_{\omega}(S)$ with respect to $\omega$ consists of all left $S$-modules $N$ satisfying the following conditions.

(A1) $N \in \omega_{S}^{\top}$.

(A2) $\omega \otimes_{S} N \in{ }_{R} \omega^{\perp}$.

(A3) $\mu_{N}$ is an isomorphism in $\operatorname{Mod} S$.

(2) The Bass class $\mathcal{B}_{\omega}(R)$ with respect to $\omega$ consists of all left $R$-modules $M$ satisfying the following conditions.

(B1) $M \in{ }_{R} \omega^{\perp}$.

(B2) $M_{*} \in \omega_{S}^{\top}$.

(B3) $\theta_{M}$ is an isomorphism in $\operatorname{Mod} R$.

For a module $M \in \operatorname{Mod} R$, we use

$$
0 \rightarrow M \rightarrow I^{0}(M) \stackrel{g^{0}}{\longrightarrow} I^{1}(M) \stackrel{g^{1}}{\longrightarrow} \cdots \stackrel{g^{i-1}}{\longrightarrow} I^{i}(M) \stackrel{g^{i}}{\longrightarrow} \cdots
$$

to denote the minimal injective resolution of $M$. For any $n \geqslant 1, \operatorname{co} \Omega^{n}(M):=$ $\operatorname{Im} g^{n-1}$ is called the $n$-cosyzygy of $M$; in particular, $\operatorname{co} \Omega^{0}(M)=M$. We use $\operatorname{co} \Omega^{n}(R)$ to denote the subclass of $\operatorname{Mod} R$ consisting of $n$-cosyzygy modules. Symmetrically, $\operatorname{co} \Omega^{n}\left(S^{o p}\right)$ is defined.

Definition 2.3. ([32]). Let $M \in \operatorname{Mod} R$ and $n \geqslant 1$.

(1) $c \operatorname{Tr}_{\omega} M:=\operatorname{Coker}\left(g^{0}{ }_{*}\right)$ is called the cotranspose of $M$ with respect to ${ }_{R} \omega_{S}$, where $g^{0}$ is as in (2.1).

(2) $M$ is called $n$ - $\omega$-cotorsionfree if $\operatorname{cTr}_{\omega} M \in \omega_{S}{ }^{T_{n}}$; and is called $\infty$ - $\omega$-cotorsionfree if it is $n$ - $\omega$-cotorsionfree for all $n$.

We use $c \mathcal{T}_{\omega}^{n}(R)$ (resp. $\left.\quad c \mathcal{T}_{\omega}(R)\right)$ to denote the subclass of Mod $R$ consisting of $n$ - $\omega$-cotorsionfree modules (resp. $\infty$ - $\omega$-cotorsionfree modules). Symmetrically, $c \mathcal{T}_{\omega}^{n}\left(S^{o p}\right)$ is defined. By [32, Proposition 3.2], we have that a module in $\operatorname{Mod} R$ is $\omega$-cotorsionless (resp. $\omega$-coreflexive) if and only if it is in $\mathrm{c} \mathcal{T}_{\omega}^{1}(R)\left(\operatorname{resp} . \mathrm{c} \mathcal{T}_{\omega}^{2}(R)\right)$.

Recall from [13] that a homomorphism $f: F \rightarrow N$ in $\operatorname{Mod} S$ with $F$ flat is called a flat cover of $N$ if $\operatorname{Hom}_{S}\left(F^{\prime}, f\right)$ is epic for any flat module $F^{\prime}$ in $\operatorname{Mod} S$, and an endomorphism $h: F \rightarrow F$ is an automorphism whenever $f=f h$. Let $N \in \operatorname{Mod} S$. Bican, El Bashir and Enochs proved in 9 that $N$ has a flat cover. We use

$$
\cdots \stackrel{f_{n}}{\longrightarrow} F_{n}(N) \stackrel{f_{n-1}}{\longrightarrow} \cdots \stackrel{f_{1}}{\longrightarrow} F_{1}(N) \stackrel{f_{0}}{\longrightarrow} F_{0}(N) \rightarrow N \rightarrow 0
$$

to denote the minimal flat resolution of $N$ in $\operatorname{Mod} S$, where each $F_{i}(N) \rightarrow$ Coker $f_{i}$ is a flat cover of Coker $f_{i}$. For any $n \geqslant 1, \Omega_{\mathcal{F}}^{n}(N):=\operatorname{Im} f_{n-1}$ is called the $n$-yoke of $N$; in particular, $\Omega_{\mathcal{F}}^{0}(N)=N$. We use $\Omega_{\mathcal{F}}^{n}(S)$ to denote the subclass of $\operatorname{Mod} S$ consisting of $n$-yoke modules. Symmetrically, $\Omega_{\mathcal{F}}^{n}\left(R^{o p}\right)$ is defined.

Definition 2.4. ([34]) Let $N \in \operatorname{Mod} S$ and $n \geqslant 1$. 
(1) $\operatorname{ac}_{\omega} \operatorname{Tr}_{\omega}:=\operatorname{Ker}\left(1_{\omega} \otimes f_{0}\right)$ is called the adjoint cotranspose of $N$ with respect to ${ }_{R} \omega_{S}$, where $f_{0}$ is as in (2.2).

(2) $N$ is called adjoint $n$ - $\omega$-cotorsionfree if $\operatorname{acTr}_{\omega} N \in{ }_{R} \omega^{\perp_{n}}$; and is called adjoint $\infty$ - $\omega$-cotorsionfree if it is adjoint $n$ - $\omega$-cotorsionfree for all $n$.

We use ac $\mathcal{T}_{\omega}^{n}(S)$ (resp. ac $\left.\mathcal{T} \omega(S)\right)$ to denote the subclass of Mod $S$ consisting of adjoint $n$ - $\omega$-cotorsionfree modules (resp. adjoint $\infty$ - $\omega$-cotorsionfree modules). Symmetrically, ac $\mathcal{T}_{\omega}^{n}\left(R^{o p}\right)$ is defined. By [34, Proposition 3.2], we have that a module in $\operatorname{Mod} S$ is adjoint $\omega$-cotorsionless (resp. adjoint $\omega$-coreflexive) if and only if it is in $\operatorname{ac}_{\omega}^{1}(S)\left(\operatorname{resp} . \operatorname{ac} \mathcal{T}_{\omega}^{2}(S)\right)$.

Definition 2.5. (33]

(1) Let $M \in \operatorname{Mod} R$ and $n \geqslant 0$. The Ext-cograde of $M$ with respect to $\omega$ is defined as E-cograde $\omega:=\inf \left\{i \geqslant 0 \mid \operatorname{Ext}_{R}^{i}(\omega, M) \neq 0\right\}$; and the strong Ext-cograde of $M$ with respect to $\omega$, denoted by s.E-cograde $\omega$, is said to be at least $n$ if E-cograde ${ }_{\omega} X \geqslant n$ for any quotient module $X$ of $M$. Symmetrically, the (strong) Ext-cograde of a module in Mod $S^{o p}$ is defined.

(2) Let $N \in \operatorname{Mod} S$ and $n \geqslant 0$. The Tor-cograde of $N$ with respect to $\omega$ is defined as T-cograde $\omega:=\inf \left\{i \geqslant 0 \mid \operatorname{Tor}_{i}^{S}(\omega, N) \neq 0\right\}$; and the strong Tor-cograde of $N$ with respect to $\omega$, denoted by s.T-cograde $\omega$, is said to be at least $n$ if T-cograde ${ }_{\omega} Y \geqslant n$ for any submodule $Y$ of $N$. Symmetrically, the (strong) Tor-cograde of a module in $\operatorname{Mod} R^{o p}$ is defined.

Let $\mathcal{X}$ be a subclass of $\operatorname{Mod} R$ and $M \in \operatorname{Mod} R$. An exact sequence (of finite or infinite length):

$$
\cdots \rightarrow X_{n} \rightarrow \cdots \rightarrow X_{1} \rightarrow X_{0} \rightarrow M \rightarrow 0
$$

in $\operatorname{Mod} R$ is called an $\mathcal{X}$-resolution of $M$ if all $X_{i}$ are in $\mathcal{X}$. The $\mathcal{X}$-projective dimension $\mathcal{X}-\operatorname{pd}_{R} M$ of $M$ is defined as $\inf \{n \mid$ there exists an $\mathcal{X}$-resolution

$$
0 \rightarrow X_{n} \rightarrow \cdots \rightarrow X_{1} \rightarrow X_{0} \rightarrow M \rightarrow 0
$$

of $M$ in $\operatorname{Mod} R\}$. Dually, the notions of an $\mathcal{X}$-coresolution and the $\mathcal{X}$-injective dimension $\mathcal{X}-\operatorname{id}_{R} M$ of $M$ are defined.

Let $\mathcal{F}$ be a subclass of $\operatorname{Mod} R$. A module $M \in \operatorname{Mod} R$ is said to have special $\mathcal{F}$-precover if there exists an exact sequence

$$
0 \rightarrow K \rightarrow F \rightarrow M \rightarrow 0
$$

in $\operatorname{Mod} R$ with $F \in \mathcal{F}$ and $\operatorname{Ext}_{R}^{1}\left(F^{\prime}, K\right)=0$ for any $F^{\prime} \in \mathcal{F}$. The class $\mathcal{F}$ is called special precovering if any module in $\operatorname{Mod} R$ has a special $\mathcal{F}$-precover. Dually, the notions of special $\mathcal{F}$-preenvelopes and special preenveloping classes are defined (see 14).

Definition 2.6. (cf. [17) Let $\mathcal{U}, \mathcal{V}$ be subclasses of $\operatorname{Mod} R$. The pair $(\mathcal{U}, \mathcal{V})$ is called a cotorsion pair if $\mathcal{U}={ }^{\perp_{1}} \mathcal{V}:=\left\{U \in \operatorname{Mod} R \mid \operatorname{Ext}_{R}^{1}(U, V)=0\right.$ for any $V \in \mathcal{V}\}$ and $\mathcal{V}=\mathcal{U}^{\perp_{1}}:=\left\{V \in \operatorname{Mod} R \mid \operatorname{Ext}_{R}^{1}(U, V)=0\right.$ for any $\left.U \in \mathcal{U}\right\}$.

The following is the Salce's lemma.

Lemma 2.7. (cf. [17, Lemma 2.2.6]) Let $(\mathcal{U}, \mathcal{V})$ be a cotorsion pair in $\operatorname{Mod} R$. Then the following statements are equivalent.

(1) Any module in $\operatorname{Mod} R$ has a special $\mathcal{U}$-precover.

(2) Any module in $\operatorname{Mod} R$ has a special $\mathcal{V}$-preenvelope. 
In this case, the cotorsion pair $(\mathcal{U}, \mathcal{V})$ is called complete.

Definition 2.8. Let $\mathcal{X}$ be a subcategory of an abelian category $\mathcal{E}$ and $n \geqslant 1$. If there exists an exact sequence

$$
0 \rightarrow N \rightarrow X_{0} \rightarrow \cdots \rightarrow X_{n-1} \rightarrow M \rightarrow 0
$$

in $\mathcal{E}$ with all $X_{i}$ in $\mathcal{X}$, then $N$ is called an $n$-X $\mathcal{X}$-syzygy of $M$ and $M$ is called an $n-\mathcal{X}$-cosyzygy of $N$.

For subcategories $\mathcal{X}, \mathcal{Y}$ of an abelian category $\mathcal{E}$ and $n \geqslant 1$, we write

$$
\begin{aligned}
& \Omega_{\mathcal{X}}^{n}(\mathcal{Y}):=\{N \in \mathcal{A} \mid N \text { is an } n \text { - } \mathcal{X} \text {-syzygy of some object in } \mathcal{Y}\}, \\
& \operatorname{co} \Omega_{\mathcal{X}}^{n}(\mathcal{Y}):=\{M \in \mathcal{A} \mid M \text { is an } n \text { - } \mathcal{X} \text {-cosyzygy of some object in } \mathcal{Y}\} \text {. }
\end{aligned}
$$

In particular, $\Omega_{\mathcal{X}}^{0}(\mathcal{Y})=\mathcal{Y}=\cos \Omega_{\mathcal{X}}^{0}(\mathcal{Y})$ and $\Omega_{\mathcal{X}}^{-1}(\mathcal{Y})=0=\operatorname{co} \Omega_{\mathcal{X}}^{-1}(\mathcal{Y})$. For convenience, we write

$$
\begin{gathered}
\Omega_{\mathcal{A}}^{n}(S):=\Omega_{\mathcal{A}_{\omega}(S)}^{n}(\operatorname{Mod} S), \Omega_{\mathcal{I}_{\omega}}^{n}(S):=\Omega_{\mathcal{I}_{\omega}(S)}^{n}(\operatorname{Mod} S), \\
\Omega_{\mathcal{I}_{\omega}}^{n}\left(R^{o p}\right):=\Omega_{\mathcal{I}_{\omega}\left(R^{o p}\right)}^{n}\left(\operatorname{Mod} R^{o p}\right), \\
\operatorname{co} \Omega_{\mathcal{B}}^{n}(R):=\cos \Omega_{\mathcal{B}_{\omega}(R)}^{n}(\operatorname{Mod} R), \cos \Omega_{\mathcal{F}_{\omega}}^{n}(R):=\cos \Omega_{\mathcal{F}_{\omega}(R)}^{n}(\operatorname{Mod} R), \\
\operatorname{co} \Omega_{\mathcal{P}_{\omega}}^{n}(R):=\operatorname{co} \Omega_{\mathcal{P}_{\omega}(R)}^{n}(\operatorname{Mod} R), \operatorname{co} \Omega_{\mathcal{P}_{\omega}}^{n}\left(S^{o p}\right):=\operatorname{co} \Omega_{\mathcal{P}_{\omega}\left(S^{o p}\right)}^{n}\left(\operatorname{Mod} S^{o p}\right) .
\end{gathered}
$$

Lemma 2.9. We have

(1) $\Omega_{\mathcal{I}_{\omega}}^{1}(S)=\operatorname{ac} \mathcal{T}_{\omega}^{1}(S)$.

(2) $\operatorname{co} \Omega_{\mathcal{P}_{\omega}}^{1}(R)=\mathrm{c} \mathcal{T}_{\omega}^{1}(R)$.

Proof. (1) By [34, Proposition 3.8], we have $\operatorname{ac}^{1} \mathcal{T}_{\omega}(S) \subseteq \Omega_{\mathcal{I}_{\omega}}^{1}(S)$. Now let $N \in$ $\Omega_{\mathcal{I}_{\omega}}^{1}(S)$ and let $f^{0}: N \longmapsto I^{0}$ be a monomorphism in $\operatorname{Mod} S$ with $I^{0} \in \mathcal{I}_{\omega}(S)$. Then from the following commutative diagram

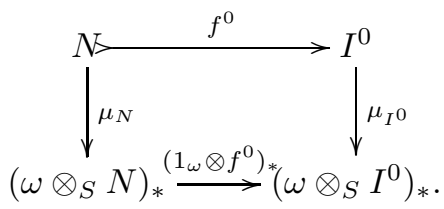

with $\mu_{I^{0}}$ an isomorphism, we get that $\mu_{N}$ is a monomorphism and $N \in \operatorname{ac} \mathcal{T}_{\omega}^{1}(S)$. It implies $\Omega_{\mathcal{I}_{\omega}}^{1}(S) \subseteq \operatorname{ac} \mathcal{T}_{\omega}^{1}(S)$.

(2) By [32, Proposition 3.7], we have $\mathrm{c} \mathcal{T}_{\omega}^{1}(R) \subseteq \operatorname{co} \Omega_{\mathcal{P}_{\omega}}^{1}(R)$. Now let $M \in$ $\cos \Omega_{\mathcal{P}_{\omega}}^{1}(R)$ and let $f_{0}: W_{0} \rightarrow M$ be an epimorphism in $\operatorname{Mod} R$ with $W_{0} \in \mathcal{P}_{\omega}(R)$. Then from the following commutative diagram

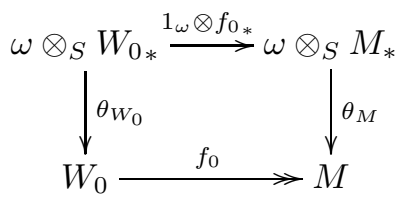

with $\theta_{W_{0}}$ an isomorphism, we get that $\theta_{M}$ is an epimorphism and $M \in \mathrm{c} \mathcal{T}_{\omega}^{1}(R)$. It implies $\cos \Omega_{\mathcal{P}_{\omega}}^{1}(R) \subseteq \mathrm{c} \mathcal{T}_{\omega}^{1}(R)$.

Let $\mathcal{C}, \mathcal{E}$ be abelian categories and $\Delta: \mathcal{C} \rightarrow \mathcal{E}$ a functor. Recall that a sequence $\mathbb{T}$ in $\mathcal{C}$ is called $\Delta$-exact if $\Delta(\mathbb{T})$ is exact in $\mathcal{E}$. 


\section{3. (Strong) cograde conditions and double homological functors}

In this section, we study when $\operatorname{Tor}_{i}^{S}\left(\omega, \operatorname{Ext}_{R}^{i}(\omega,-)\right)$ preserves epimorphisms and $\operatorname{Ext}_{S^{o p}}^{i}\left(\omega, \operatorname{Tor}_{i}^{R}(-, \omega)\right)$ preserves monomorphisms in terms of the (strong) cograde conditions of modules.

\subsection{Cograde conditions}

We begin with the following

\section{Lemma 3.1.}

(1) Let $M \in \operatorname{Mod} R$ with the minimal injective resolution as (2.1). Then there exists an exact sequence

$$
0 \rightarrow \operatorname{Ext}_{R}^{1}(\omega, M) \stackrel{\lambda}{\longrightarrow} \operatorname{cTr}_{\omega} M \stackrel{\pi}{\longrightarrow} I^{1}(M)_{*} / \operatorname{co} \Omega^{1}(M)_{*} \rightarrow 0
$$

in $\operatorname{Mod} S$ such that $1_{\omega} \otimes \pi$ is an isomorphism.

(2) Let $N \in \operatorname{Mod} S$ with the minimal flat resolution as (2.2). Then there exists an exact sequence

$$
0 \rightarrow \operatorname{Im}\left(1_{\omega} \otimes f_{1}\right) \stackrel{\sigma}{\longrightarrow} \operatorname{ac} \operatorname{Tr}_{\omega} N \stackrel{\tau}{\longrightarrow} \operatorname{Tor}_{1}^{S}(\omega, N) \rightarrow 0
$$

in $\operatorname{Mod} R$ such that $\sigma_{*}$ is an isomorphism.

Proof. (1) Let $g^{0}=\alpha \cdot \beta$ (where $\beta: I^{0}(M) \rightarrow \operatorname{co} \Omega^{1}(M)\left(=\operatorname{Im} g^{0}\right.$ ) and $\alpha$ : $\left.\operatorname{co} \Omega^{1}(M) \longmapsto I^{1}(M)\right)$ be the natural epic-monic decomposition of $g^{0}$. Then we have the following commutative diagram with exact columns and rows

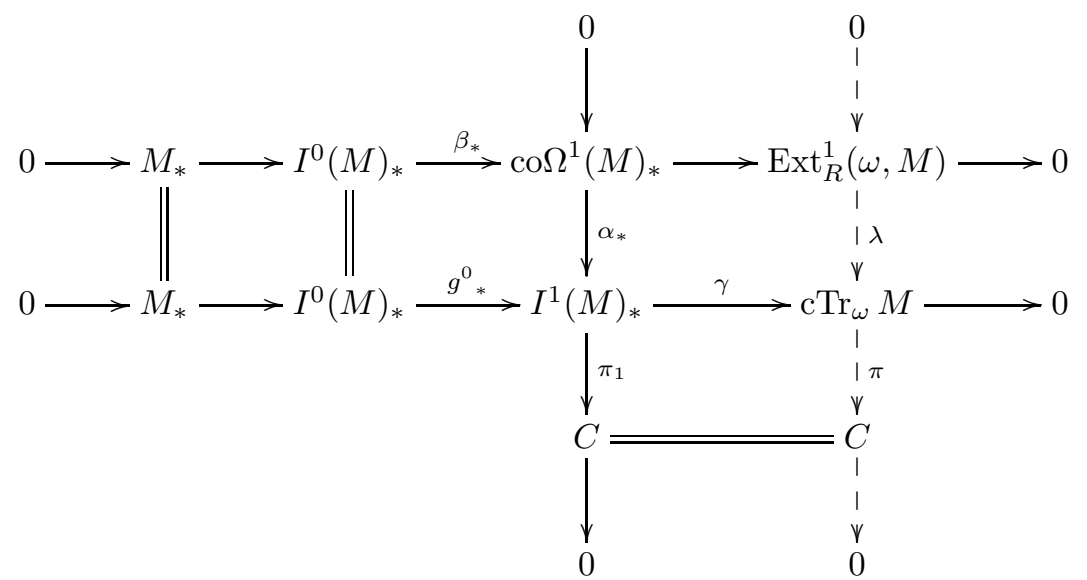

in $\operatorname{Mod} S$, where $C=I^{1}(M)_{*} / \operatorname{co} \Omega^{1}(M)_{*}, \pi_{1}$ is the natural epimorphism, $\lambda$ and $\pi$ are induced homomorphisms. The rightmost column in the above diagram is exactly the exact sequence (3.1). Notice that

$$
0 \rightarrow \operatorname{co} \Omega^{1}(M)_{*} \stackrel{\alpha_{*}}{\longrightarrow} I^{1}(M)_{*} \stackrel{g^{1} *}{\longrightarrow} I^{2}(M)_{*}
$$

is exact, so there exists a homomorphism $\delta: C \rightarrow I^{2}(M)_{*}$ in $\operatorname{Mod} S$ such that $g^{1}=\delta \cdot \pi_{1}$, and hence $g_{*}^{1}=\delta \cdot \pi_{1}=\delta \cdot \pi \cdot \gamma$. 
By [19, Lemma 4.1], for any injective module $I \in \operatorname{Mod} R$, we have $\omega \otimes_{S} I_{*} \cong I$ canonically. So the upper row in the following commutative diagram

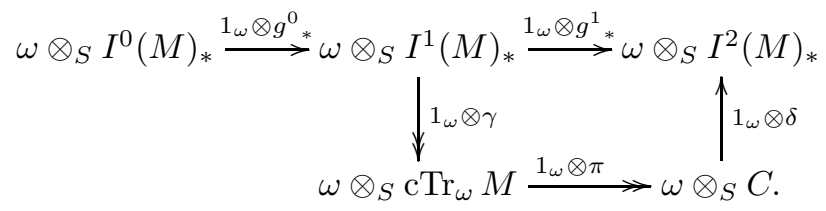

is exact. Let $x \in \operatorname{Ker}\left(1_{\omega} \otimes \pi\right)$. Then there exists $y \in \omega \otimes_{S} I^{1}(M)_{*}$ such that $x=\left(1_{\omega} \otimes \gamma\right)(y)$. It follows that

$$
\left(1_{\omega} \otimes g_{*}^{1}\right)(y)=\left(1_{\omega} \otimes \delta\right) \cdot\left(1_{\omega} \otimes \pi\right) \cdot\left(1_{\omega} \otimes \gamma\right)(y)=\left(1_{\omega} \otimes \delta\right) \cdot\left(1_{\omega} \otimes \pi\right)(x)=0 .
$$

So $y \in \operatorname{Ker}\left(1_{\omega} \otimes g^{1}{ }_{*}\right)=\operatorname{Im}\left(1_{\omega} \otimes g^{0}{ }_{*}\right)$, and hence there exists $z \in \omega \otimes_{S} I^{0}(M)_{*}$ such that $y=\left(1_{\omega} \otimes g_{*}^{0}\right)(z)$. Thus

$$
x=\left(1_{\omega} \otimes \gamma\right)(y)=\left(1_{\omega} \otimes \gamma\right) \cdot\left(1_{\omega} \otimes g_{*}^{0}\right)(z)=\left(1_{\omega} \otimes\left(\gamma \cdot g_{*}^{0}\right)\right)(z)=0,
$$

which implies that $1_{\omega} \otimes \pi$ is a monomorphism, and hence an isomorphism.

(2) Let $f_{0}=\alpha^{\prime} \cdot \beta^{\prime}\left(\right.$ where $\beta^{\prime}: F_{1}(N) \rightarrow \Omega_{\mathcal{F}}^{1}(N)\left(=\operatorname{Im} f_{0}\right)$ and $\alpha^{\prime}: \Omega_{\mathcal{F}}^{1}(N) \longmapsto$ $F_{0}(N)$ ) be the natural epic-monic decomposition of $f_{0}$. Then we have the following commutative diagram with exact columns and rows

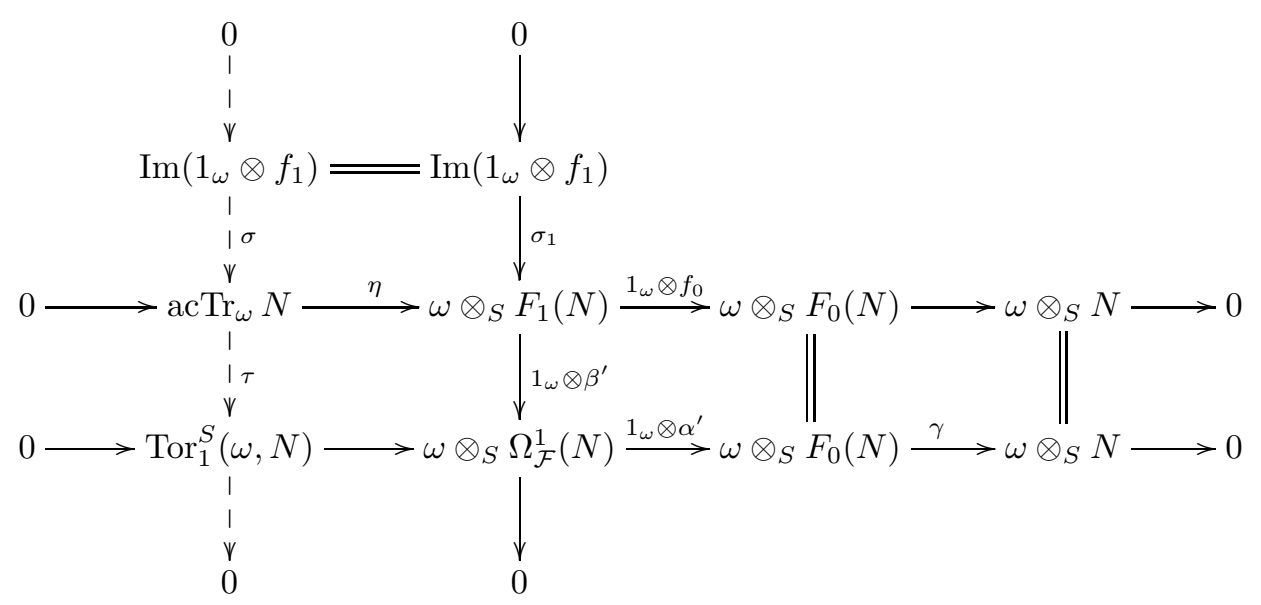

in $\operatorname{Mod} R$, where $\sigma$ and $\tau$ are induced homomorphisms. The leftmost column in the above diagram is exactly the exact sequence (3.2). Notice that

$$
\omega \otimes_{S} F_{2}(N) \stackrel{1_{\omega} \otimes f_{1}}{\longrightarrow} \omega \otimes_{S} F_{1}(N) \stackrel{1_{\omega} \otimes \beta^{\prime}}{\longrightarrow} \omega \otimes_{S} \Omega_{\mathcal{F}}^{1}(N) \rightarrow 0
$$

is exact, so there exists a homomorphism $\phi: \omega \otimes_{S} F_{2}(N) \rightarrow \operatorname{Im}\left(1_{\omega} \otimes f_{1}\right)$ in $\operatorname{Mod} R$ such that $1_{\omega} \otimes f_{1}=\sigma_{1} \cdot \phi$, and hence $1_{\omega} \otimes f_{1}=\sigma_{1} \cdot \phi=\eta \cdot \sigma \cdot \phi$.

By [19, Lemma 4.1], for any flat module $F \in \operatorname{Mod} S$, we have $F \cong\left(\omega \otimes_{S} F\right)_{*}$ canonically. So the upper row in the following commutative diagram is exact.

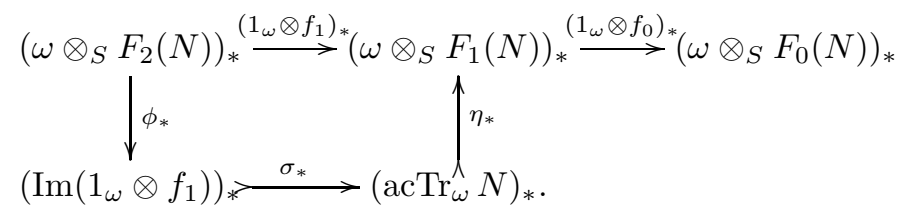


Let $x \in\left(\operatorname{acTr}_{\omega} N\right)_{*}$. Since $\left(\left(1_{\omega} \otimes f_{0}\right)_{*} \cdot \eta_{*}\right)(x)=\left(\left(\left(1_{\omega} \otimes f_{0}\right) \cdot \eta\right)_{*}\right)(x)=0$, we have that $\eta_{*}(x) \in \operatorname{Ker}\left(1_{\omega} \otimes f_{0}\right)_{*}=\operatorname{Im}\left(1_{\omega} \otimes f_{1}\right)_{*}$ and there exists $y \in\left(\omega \otimes_{S} F_{2}(N)\right)_{*}$ such that $\eta_{*}(x)=\left(1_{\omega} \otimes f_{1}\right)_{*}(y)$. Thus

$$
\eta_{*}(x)=\left(1_{\omega} \otimes f_{1}\right)_{*}(y)=\left(\eta_{*} \cdot \sigma_{*} \cdot \phi_{*}\right)(y) .
$$

As $\eta^{*}$ is monic, we have $x=\sigma_{*}\left(\phi_{*}(y)\right)$. It means that $\sigma_{*}$ is an epimorphism, and hence an isomorphism.

The following two lemmas are useful in this section.

Lemma 3.2. Assume that $\cos \Omega^{n}(R) \subseteq \mathrm{c} \mathcal{T}_{\omega}^{m}(R)$ with $m, n \geqslant 0$. Then the following statements are equivalent.

(1) T-cograde $\operatorname{Ext}_{R}^{n+1}(\omega, M) \geqslant m$ for any $M \in \operatorname{Mod} R$.

(2) $\cos \Omega^{n+1}(R) \subseteq \mathrm{c} \mathcal{T}_{\omega}^{m+1}(R)$.

Proof. Because any injective module in $\operatorname{Mod} R$ is in $\mathcal{c}^{1}(R)$ by [32, Lemma 2.5(2)], we have that $\cos \Omega^{n+1}(R) \subseteq \mathrm{c} \mathcal{T}_{\omega}^{1}(R)$ for any $n \geqslant 0$, and the case for $m=0$ follows. Now suppose that $m \geqslant 1$ and $M \in \operatorname{Mod} R$. By Lemma 3.1(1), there exists an exact sequence

$$
0 \rightarrow \operatorname{Ext}_{R}^{1}\left(\omega, \cos \Omega^{n}(M)\right) \stackrel{\lambda}{\longrightarrow} \operatorname{cTr}_{\omega} \cos \Omega^{n}(M) \stackrel{\pi}{\longrightarrow} C \rightarrow 0
$$

in Mod $S$ such that $1_{\omega} \otimes \pi$ is an isomorphism, where $C=I^{n+1}(M)_{*} / \cos \Omega^{n+1}(M)_{*}$. Because $\operatorname{co} \Omega^{n}(R) \subseteq \mathrm{c} \mathcal{T}_{\omega}^{m}(R)$ by assumption, we have that both $\operatorname{cTr}_{\omega} \operatorname{co} \Omega^{n}(M)$ and $c \operatorname{Tr}_{\omega} \cos \Omega^{n+1}(M)$ are in $\omega_{S}^{T_{m}}$. It yields that

$$
\operatorname{Tor}_{i}^{S}\left(\omega, \operatorname{Ext}_{R}^{n+1}(\omega, M)\right) \cong \operatorname{Tor}_{i}^{S}\left(\omega, \operatorname{Ext}_{R}^{1}\left(\omega, \cos ^{n}(M)\right)\right) \cong \operatorname{Tor}_{i+1}^{S}(\omega, C)
$$

for any $0 \leqslant i \leqslant m-1$. In addition, we also have an exact sequence

$$
0 \rightarrow C \rightarrow I^{n+2}(M)_{*} \rightarrow \mathrm{cTr}_{\omega} \operatorname{co} \Omega^{n+1}(M) \rightarrow 0
$$

in $\operatorname{Mod} S$. By [19, Corollary 6.1], we have $I^{n+2}(M)_{*} \in \omega_{S}^{\top}$. So

$$
\operatorname{Tor}_{i}^{S}\left(\omega, \operatorname{Ext}_{R}^{n+1}(\omega, M)\right) \cong \operatorname{Tor}_{i+1}^{S}(\omega, C) \cong \operatorname{Tor}_{i+2}^{S}\left(\omega, \operatorname{cTr}_{\omega} \cos ^{n+1}(M)\right)
$$

for any $0 \leqslant i \leqslant m-1$. Thus we conclude that $\operatorname{Tor}_{0 \leqslant i \leqslant m-1}^{S}\left(\omega, \operatorname{Ext}_{R}^{n+1}(\omega, M)\right)=0$ if and only if $\operatorname{cTr}_{\omega} \operatorname{co} \Omega^{n+1}(M) \in \omega_{S}^{T_{m+1}}$, and if and only if $\cos \Omega^{n+1}(M) \in \mathrm{c} \mathcal{T}_{\omega}^{m+1}(R)$. The proof is finished.

Lemma 3.3. Assume that $\Omega_{\mathcal{F}}^{n}(S) \subseteq$ ac $\mathcal{T}_{\omega}^{m}(S)$ with $m, n \geqslant 0$. Then the following statements are equivalent.

(1) E-cograde $_{\omega} \operatorname{Tor}_{n+1}^{S}(\omega, N) \geqslant m$ for any $N \in \operatorname{Mod} S$.

(2) $\Omega_{\mathcal{F}}^{n+1}(S) \subseteq \operatorname{ac} \mathcal{T}_{\omega}^{m+1}(S)$.

Proof. Because any flat module in $\operatorname{Mod} S$ is in $\operatorname{ac}_{\omega}^{1}(S)$ by [34, Corollary 3.5(1)], we have that $\Omega_{\mathcal{F}}^{n+1}(S) \subseteq$ ac $\mathcal{T}_{\omega}^{1}(S)$ for any $n \geqslant 0$, and the case for $m=0$ follows. Now suppose that $m \geqslant 1$ and $N \in \operatorname{Mod} S$. By Lemma 3.1(2), there exists an exact sequence

$$
0 \rightarrow \operatorname{Im}\left(1_{\omega} \otimes f_{n+1}\right) \stackrel{\sigma}{\rightarrow} \operatorname{ac} \operatorname{Tr}_{\omega} \Omega_{\mathcal{F}}^{n}(N) \stackrel{\tau}{\rightarrow} \operatorname{Tor}_{1}^{S}\left(\omega, \Omega_{\mathcal{F}}^{n}(N)\right) \rightarrow 0
$$

in $\operatorname{Mod} R$ such that $\sigma_{*}$ is an isomorphism. Because $\Omega_{\mathcal{F}}^{n}(S) \subseteq$ ac $\mathcal{T}_{\omega}^{m}(S)$ by assumption, we have that both $\operatorname{ac} \operatorname{Tr}_{\omega} \Omega_{\mathcal{F}}^{n}(N)$ and $\operatorname{ac}_{\omega} \operatorname{Tr}_{\omega} \Omega_{\mathcal{F}}^{n+1}(N)$ are in ${ }_{R} \omega^{\perp_{m}}$. It yields that

$$
\operatorname{Ext}_{R}^{i}\left(\omega, \operatorname{Tor}_{n+1}^{S}(\omega, N)\right) \cong \operatorname{Ext}_{R}^{i}\left(\omega, \operatorname{Tor}_{1}^{S}\left(\omega, \Omega_{\mathcal{F}}^{n}(N)\right)\right) \cong \operatorname{Ext}_{R}^{i+1}\left(\omega, \operatorname{Im}\left(1_{\omega} \otimes f_{n+1}\right)\right)
$$


for any $0 \leqslant i \leqslant m-1$. In addition, we also have an exact sequence

$$
0 \rightarrow \operatorname{acTr}_{\omega} \Omega_{\mathcal{F}}^{n+1}(N) \rightarrow \omega \otimes_{S} F_{n+2}(N) \rightarrow \operatorname{Im}\left(1_{\omega} \otimes f_{n+1}\right) \rightarrow 0
$$

in $\operatorname{Mod} R$. By [19, Corollary 6.1], we have $\omega \otimes_{S} F_{n+2}(N) \in{ }_{R} \omega^{\perp}$. So

$$
\operatorname{Ext}_{R}^{i}\left(\omega, \operatorname{Tor}_{n+1}^{S}(\omega, N)\right) \cong \operatorname{Ext}_{R}^{i+1}\left(\omega, \operatorname{Im}\left(1_{\omega} \otimes f_{n+1}\right)\right) \cong \operatorname{Ext}_{R}^{i+2}\left(\omega, \operatorname{ac} \operatorname{Tr}_{\omega} \Omega_{\mathcal{F}}^{n+1}(N)\right)
$$

for any $0 \leqslant i \leqslant m-1$. Thus we conclude that $\operatorname{Ext}_{R}^{0 \leqslant i \leqslant m-1}\left(\omega, \operatorname{Tor}_{n+1}^{S}(\omega, N)\right)=0$ if and only if $\operatorname{ac}_{\operatorname{Tr}_{\omega}} \Omega_{\mathcal{F}}^{n+1}(N) \in{ }_{R} \omega^{\perp_{m+1}}$, and if and only if $\Omega_{\mathcal{F}}^{n+1}(N) \in \operatorname{ac} \mathcal{T}_{\omega}^{m+1}(S)$. The proof is finished.

Let $\mathcal{T} \subseteq \mathcal{W}$ be subcategories of an abelian category $\mathcal{E}$. Recall that $\mathcal{T}$ is called a generator (resp. cogenerator) for $\mathcal{W}$ if for any $W \in \mathcal{W}$, there exists an exact sequence

$$
0 \rightarrow W^{\prime} \rightarrow T \rightarrow W \rightarrow 0 \text { (resp. } 0 \rightarrow W \rightarrow T \rightarrow W^{\prime} \rightarrow 0 \text { ) }
$$

in $\mathcal{E}$ with $T \in \mathcal{T}$ and $W^{\prime} \in \mathcal{W}$.

\section{Lemma 3.4.}

(1) $\mathcal{P}_{\omega}(R)$ is a generator for $\mathcal{B}_{\omega}(R)$.

(2) $\operatorname{co} \Omega^{n}(R) \subseteq \operatorname{co} \Omega_{\mathcal{B}}^{n}(R)=\operatorname{co} \Omega_{\mathcal{F}_{\omega}}^{n}(R)=\operatorname{co} \Omega_{\mathcal{P}_{\omega}}^{n}(R)$ for any $n \geqslant 1$.

Proof. (1) Let $M \in \mathcal{B}_{\omega}(R)$. Then by [32, Theorem 3.9 and Proposition 3.7], there exists an exact sequence

$$
\cdots \rightarrow W_{2} \rightarrow W_{1} \rightarrow W_{0} \rightarrow M \rightarrow 0
$$

in $\operatorname{Mod} R$ with all $W_{i} \in \mathcal{P}_{\omega}(R)$ such that it remains exact after applying the functor $\operatorname{Hom}_{R}(\omega,-)$. Put $M_{1}:=\operatorname{Im}\left(W_{1} \rightarrow W_{0}\right)$. Then $M_{1} \in \mathrm{c} \mathcal{T}_{\omega}(R)$ by [32, Proposition 3.7]. Because both $M$ and $W_{0}$ are in ${ }_{R} \omega^{\perp}$, we have $M_{1} \in{ }_{R} \omega^{\perp}$. So $M_{1} \in \mathcal{B}_{\omega}(R)$ by 32, Theorem 3.9].

(2) Let $n \geqslant 1$. By [19, Lemma 4.1], we have that $\mathcal{B}_{\omega}(R)$ contains all injective left $R$-modules, which yields $\operatorname{co} \Omega^{n}(R) \subseteq \operatorname{co} \Omega_{\mathcal{B}}^{n}(R)$. Because $\mathcal{B}_{\omega}(R) \supseteq \mathcal{F}_{\omega}(R) \supseteq \mathcal{P}_{\omega}(R)$ by [19, Corollary 6.1], we have $\cos \Omega_{\mathcal{B}}^{n}(R) \supseteq \operatorname{co} \Omega_{\mathcal{F}_{\omega}}^{n}(R) \supseteq \operatorname{co} \Omega_{\mathcal{P}_{\omega}}^{n}(R)$. Because $\mathcal{B}_{\omega}(R)$ is closed under extensions by [19, Theorem 6.2], we have $\operatorname{co} \Omega_{\mathcal{B}}^{n}(R)=\operatorname{co} \Omega_{\mathcal{P}_{\omega}}^{n}(R)$ by (1) and [23, Corollary 5.4(2)].

In the following result, we characterize when the double functor $\operatorname{Tor}_{i}^{S}\left(\omega, \operatorname{Ext}_{R}^{i}(\omega,-)\right)$ preserves epimorphisms in terms of the Tor-cograde conditions of Ext-modules.

Theorem 3.5. The conditions (1)-(3) below are equivalent for any $n, k \geqslant 0$. If $k \geqslant 1$, then (1)-(4) are equivalent.

(1) $\mathrm{T}-\operatorname{cograde}_{\omega} \operatorname{Ext}_{R}^{i+k}(\omega, M) \geqslant i$ for any $M \in \operatorname{Mod} R$ and $1 \leqslant i \leqslant n$.

(2) $\operatorname{Tor}_{i}^{S}\left(\omega, \operatorname{Ext}_{R}^{i}(\omega, f)\right)$ is an epimorphism for any epimorphism $f: B \rightarrow C$ in $\operatorname{Mod} R$ with $B, C \in \operatorname{co} \Omega_{\mathcal{P}_{\omega}}^{k+1}(R)$ and $0 \leqslant i \leqslant n-1$.

(3) $\operatorname{Tor}_{i}^{S}\left(\omega, \operatorname{Ext}_{R}^{i}(\omega, f)\right)$ is an epimorphism for any epimorphism $f: B \rightarrow C$ in $\operatorname{Mod} R$ with $B, C \in \operatorname{co} \Omega^{k+1}(R)$ and $0 \leqslant i \leqslant n-1$.

(4) $\cos \Omega^{i+k}(R) \subseteq \mathrm{c} \mathcal{T}_{\omega}^{i+1}(R)$ for any $1 \leqslant i \leqslant n$.

Proof. By using induction on $i,(1) \Leftrightarrow$ (4) follows from Lemma 3.2

$(1) \Rightarrow(2)$ Let $f: B \rightarrow C$ be an epimorphism in $\operatorname{Mod} R$ with $B, C \in \cos _{\mathcal{P}_{\omega}}^{k+1}(R)$. Then $C=\operatorname{co} \Omega_{\mathcal{P}_{\omega}}^{k+1}\left(C^{\prime}\right)$ for some $C^{\prime} \in \operatorname{Mod} R$. By (1), we have

$$
\operatorname{Tor}_{i}^{S}\left(\omega, \operatorname{Ext}_{R}^{i}(\omega, C)\right) \cong \operatorname{Tor}_{i}^{S}\left(\omega, \operatorname{Ext}_{R}^{i+k+1}\left(\omega, C^{\prime}\right)\right)=0
$$


for any $1 \leqslant i \leqslant n-1$. Thus $\operatorname{Tor}_{i}^{S}\left(\omega, \operatorname{Ext}_{R}^{i}(\omega, f)\right)$ is epic. In the following, we will show that $1_{\omega} \otimes f_{*}$ is epic.

If $k \geqslant 1$, then $\cos \Omega_{\mathcal{P}_{\omega}}^{k}(R) \subseteq \mathrm{c} \mathcal{T}_{\omega}^{1}(R)$ by Lemma 2.9 (2). So $\operatorname{co} \Omega_{\mathcal{P}_{\omega}}^{k+1}(R) \subseteq \mathrm{c} \mathcal{T}_{\omega}^{2}(R)$ by Lemma 3.2, and hence $B, C \in \mathrm{c} \mathcal{T}_{\omega}^{2}(R)$. It follows that $1_{\omega} \otimes f_{*} \cong f$ and $1_{\omega} \otimes f_{*}$ is epic.

Now suppose $k=0$. We have an epimorphism $p: W \rightarrow B$ in $\operatorname{Mod} R$ with $W \in \operatorname{Add}_{R} \omega$. From the exact sequence

$$
0 \rightarrow M_{1} \rightarrow W \stackrel{f \cdot p}{\longrightarrow} C \rightarrow 0
$$

in $\operatorname{Mod} R$ with $M_{1}=\operatorname{Ker}(f \cdot p)$, we get the following exact sequence

$$
W_{*} \stackrel{(f \cdot p)}{\longrightarrow} C_{*} \rightarrow \operatorname{Ext}_{R}^{1}\left(\omega, M_{1}\right) \rightarrow 0
$$

in $\operatorname{Mod} S$. By (1). $\omega \otimes_{S} \operatorname{Ext}_{R}^{1}\left(\omega, M_{1}\right)=0$. So $\left(1_{\omega} \otimes f_{*}\right) \cdot\left(1_{\omega} \otimes p_{*}\right)=1_{\omega} \otimes(f \cdot p)_{*}$ is epic, which implies that $1_{\omega} \otimes f_{*}$ is also epic.

By Lemma 3.4(2), we have $(2) \Rightarrow(3)$.

(3) $\Rightarrow(1)$ Let $M \in \operatorname{Mod} R$. From the exact sequence

$$
0 \rightarrow \operatorname{co} \Omega^{k}(M) \rightarrow I^{k}(M) \stackrel{f}{\longrightarrow} \cos \Omega^{k+1}(M) \rightarrow 0
$$

in $\operatorname{Mod} R$, we get the following exact sequence

$$
I^{k}(M)_{*} \stackrel{f_{*}}{\longrightarrow} \operatorname{co} \Omega^{k+1}(M)_{*} \rightarrow \operatorname{Ext}_{R}^{k+1}(\omega, M) \rightarrow 0
$$

in Mod $S$. Since $1_{\omega} \otimes f_{*}$ is an epimorphism by (2), we have that $\omega \otimes_{S} \operatorname{Ext}_{R}^{k+1}(\omega, M)=$ 0 and T-cograde ${ }_{\omega} \operatorname{Ext}_{R}^{k+1}(\omega, M) \geqslant 1$. In addition, for any $1 \leqslant i \leqslant n-1$,

$$
0=\operatorname{Tor}_{i}^{S}\left(\omega, \operatorname{Ext}_{R}^{i}\left(\omega, I^{k}(M)\right)\right) \stackrel{\operatorname{Tor}_{i}^{S}\left(\omega, \operatorname{Ext}_{R}^{i}(\omega, f)\right)}{\longrightarrow} \operatorname{Tor}_{i}^{S}\left(\omega, \operatorname{Ext}_{R}^{i}\left(\omega, \operatorname{co}^{k+1}(M)\right)\right)
$$

is epic by (3), so we have

$$
\operatorname{Tor}_{i}^{S}\left(\omega, \operatorname{Ext}_{R}^{i+k+1}(\omega, M)\right) \cong \operatorname{Tor}_{i}^{S}\left(\omega, \operatorname{Ext}_{R}^{i}\left(\omega, \cos ^{k+1}(M)\right)\right)=0 .
$$

Thus we conclude that T-cograde $\operatorname{Ext}_{R}^{i+k+1}(\omega, M) \geqslant i+1$ for any $0 \leqslant i \leqslant n-1$.

\section{Lemma 3.6.}

(1) $\mathcal{I}_{\omega}(S)$ is a cogenerator for $\mathcal{A}_{\omega}(S)$.

(2) $\Omega_{\mathcal{F}}^{n}(S) \subseteq \Omega_{\mathcal{A}}^{n}(S)=\Omega_{\mathcal{I}_{\omega}}^{n}(S)$ for any $n \geqslant 1$.

Proof. (1) Let $N \in \mathcal{A}_{\omega}(S)$. Then by [34, Theorem 3.11(1)], there exists an $\left(\omega \otimes_{S}-\right)$ exact exact sequence

$$
0 \rightarrow N \rightarrow U^{0} \rightarrow U^{1} \rightarrow U^{2} \rightarrow \cdots
$$

in $\operatorname{Mod} S$ with all $U^{i} \in \mathcal{I}_{\omega}(S)$. Put $N^{1}:=\operatorname{Im}\left(U^{0} \rightarrow U^{1}\right)$. Then $N^{1} \in \operatorname{ac} \mathcal{T}_{\omega}(S)$ by [34, Corollary 3.9]. Because both $N$ and $U^{0}$ are in $\omega_{S}{ }^{\top}$, we have $N^{1} \in \omega_{S}{ }^{\top}$. So $N^{1} \in \mathcal{A}_{\omega}(S)$ by [34, Theorem 3.11(1)] again.

(2) Let $n \geqslant 1$. By [19, Lemma 4.1], we have that $\mathcal{A}_{\omega}(S)$ contains all flat left $S$ modules, which yields $\Omega_{\mathcal{F}}^{n}(S) \subseteq \Omega_{\mathcal{A}}^{n}(S)$. Because $\mathcal{A}_{\omega}(S)$ is closed under extensions by [19, Theorem 6.2], we have $\Omega_{\mathcal{A}}^{n}(S)=\Omega_{\mathcal{I}_{\omega}}^{n}(S)$ by (1) and [23, Corollary 5.4(1)].

In the following result, we characterize when the double functor $\operatorname{Ext}_{R}^{i}\left(\omega, \operatorname{Tor}_{i}^{S}(\omega,-)\right)$ preserves monomorphisms in terms of the Ext-cograde conditions of Tor-modules. 
Theorem 3.7. The conditions (1)-(3) below are equivalent for any $n, k \geqslant 0$. If $k \geqslant 1$, then (1)-(4) are equivalent.

(1) E-cograde $_{\omega} \operatorname{Tor}_{i+k}^{S}(\omega, N) \geqslant i$ for any $N \in \operatorname{Mod} S$ and $1 \leqslant i \leqslant n$.

(2) $\operatorname{Ext}_{R}^{i}\left(\omega, \operatorname{Tor}_{i}^{S}(\omega, g)\right)$ is a monomorphism for any monomorphism $g: B^{\prime} \longmapsto$ $C^{\prime}$ in $\operatorname{Mod} S$ with $B^{\prime}, C^{\prime} \in \Omega_{\mathcal{I}_{\omega}}^{k+1}(S)$ and $0 \leqslant i \leqslant n-1$.

(3) $\operatorname{Ext}_{R}^{i}\left(\omega, \operatorname{Tor}_{i}^{S}(\omega, g)\right)$ is a monomorphism for any monomorphism $g: B^{\prime} \longmapsto$ $C^{\prime}$ in $\operatorname{Mod} S$ with $B^{\prime}, C^{\prime} \in \Omega_{\mathcal{F}}^{k+1}(S)$ and $0 \leqslant i \leqslant n-1$.

(4) $\Omega_{\mathcal{F}}^{i+k}(S) \subseteq \operatorname{ac}_{\omega}^{i+1}(S)$ for any $1 \leqslant i \leqslant n$.

Proof. By using induction on $i,(1) \Leftrightarrow(4)$ follows from Lemma 3.3

$(1) \Rightarrow(2)$ Let $g: B^{\prime} \longmapsto C^{\prime}$ be a monomorphism in $\operatorname{Mod} S$ with $B^{\prime}, C^{\prime} \in \Omega_{\mathcal{I}_{\omega}}^{k+1}(S)$. Then $B^{\prime}=\Omega_{\mathcal{I}_{\omega}}^{k+1}\left(B^{\prime \prime}\right)$ for some $B^{\prime \prime} \in \operatorname{Mod} S$. By (1), we have

$$
\operatorname{Ext}_{R}^{i}\left(\omega, \operatorname{Tor}_{i}^{S}\left(\omega, B^{\prime}\right)\right) \cong \operatorname{Ext}_{R}^{i}\left(\omega, \operatorname{Tor}_{i+k+1}^{S}\left(\omega, B^{\prime \prime}\right)\right)=0
$$

for any $1 \leqslant i \leqslant n-1$. Thus $\operatorname{Ext}_{R}^{i}\left(\omega, \operatorname{Tor}_{i}^{S}(\omega, g)\right)$ is a monic. In the following, we will show that $\left(1_{\omega} \otimes g\right)_{*}$ is monic.

If $k \geqslant 1$, then $\Omega_{\mathcal{I}_{\omega}}^{k}(S) \subseteq$ ac $\mathcal{T}_{\omega}^{1}(S)$ by Lemma 2.9(1). So $\Omega_{\mathcal{I}_{\omega}}^{k+1}(S) \subseteq$ ac $\mathcal{T}_{\omega}^{2}(S)$ by Lemma 3.3. and hence $B^{\prime}, C^{\prime} \in \operatorname{ac} \mathcal{T}_{\omega}^{2}(S)$. It follows that $\left(1_{\omega} \otimes g\right)_{*} \cong g$ and $\left(1_{\omega} \otimes g\right)_{*}$ is monic.

Now suppose $k=0$. We have a monomorphism $i: C^{\prime} \longmapsto U$ in $\operatorname{Mod} S$ with $U \in \mathcal{I}_{\omega}(S)$. From the exact sequence

$$
0 \rightarrow B^{\prime} \stackrel{i \cdot g}{\rightarrow} U \rightarrow L_{1} \rightarrow 0
$$

in $\operatorname{Mod} S$ with $L_{1}=\operatorname{Coker}(i \cdot g)$, we get the following exact sequence

$$
0 \rightarrow \operatorname{Tor}_{1}^{S}\left(\omega, L_{1}\right) \rightarrow \omega \otimes_{S} B^{\prime} \stackrel{1_{\omega} \otimes(i \cdot g)}{\longrightarrow} \omega \otimes_{S} U
$$

in $\operatorname{Mod} R$. By $(1),\left(\operatorname{Tor}_{1}^{S}\left(\omega, L_{1}\right)\right)_{*}=0$. So $\left(1_{\omega} \otimes i\right)_{*} \cdot\left(1_{\omega} \otimes g\right)_{*}=\left(1_{\omega} \otimes(i \cdot g)\right)_{*}$ is monic, which implies that $\left(1_{\omega} \otimes g\right)_{*}$ is also monic.

By Lemma $3.6(2)$, we have $(2) \Rightarrow(3)$.

$(3) \Rightarrow(1)$ Let $N \in \operatorname{Mod} S$. From the exact sequence

$$
0 \rightarrow \Omega_{\mathcal{F}}^{k+1}(N) \stackrel{g}{\longrightarrow} F_{k}(N) \rightarrow \Omega_{\mathcal{F}}^{k}(N) \rightarrow 0
$$

in $\operatorname{Mod} S$, we get the following exact sequence

$$
0 \rightarrow \operatorname{Tor}_{k+1}^{S}(\omega, N) \rightarrow \omega \otimes_{S} \Omega_{\mathcal{F}}^{k+1}(N) \stackrel{1_{\omega} \otimes g}{\longrightarrow} \omega \otimes_{S} F_{k}(N)
$$

in $\operatorname{Mod} R$. Since $\left(1_{\omega} \otimes g\right)_{*}$ is a monomorphism by $(2)$, we have that $\left(\operatorname{Tor}_{k+1}^{S}(\omega, N)\right)_{*}=$ 0 and E-cograde $\operatorname{Tor}_{k+1}^{S}(\omega, N) \geqslant 1$. In addition, for any $1 \leqslant i \leqslant n-1$,

$$
\operatorname{Ext}_{R}^{i}\left(\omega, \operatorname{Tor}_{i}^{S}\left(\omega, \Omega_{\mathcal{F}}^{k+1}(N)\right)\right) \stackrel{\operatorname{Ext}_{R}^{i}\left(\omega, \operatorname{Tor}_{i}^{S}(\omega, g)\right)}{\longrightarrow} \operatorname{Ext}_{R}^{i}\left(\omega, \operatorname{Tor}_{i}^{S}\left(\omega, F_{k}(N)\right)\right)=0
$$

is monic by (3), so we have

$$
\operatorname{Ext}_{R}^{i}\left(\omega, \operatorname{Tor}_{i+k+1}^{S}(\omega, N)\right) \cong \operatorname{Ext}_{R}^{i}\left(\omega, \operatorname{Tor}_{i}^{S}\left(\Omega_{\mathcal{F}}^{k+1}(\omega, N)\right)=0 .\right.
$$

Thus we conclude that E-cograde $\operatorname{cor}_{i+k+1}^{S}(\omega, N) \geqslant i+1$ for any $0 \leqslant i \leqslant n-1$.

\subsection{Strong cograde conditions}

Compare the following result with Theorem 3.5 
Theorem 3.8. For any $n \geqslant 1$ and $k \geqslant 0$, the following three statements are equivalent.

(1) s.T-cograde $\operatorname{Ext}_{R}^{i+k}(\omega, M) \geqslant i$ for any $M \in \operatorname{Mod} R$ and $1 \leqslant i \leqslant n$.

(2) For any exact sequence

$$
0 \rightarrow A \rightarrow B \stackrel{f}{\longrightarrow} C \rightarrow 0
$$

in $\operatorname{Mod} R$ with $A \in \Omega_{\mathcal{P}_{\omega}}^{i-1}\left(\operatorname{co} \Omega_{\mathcal{P}_{\omega}}^{i+k-1}(R)\right)$, $\operatorname{Tor}_{i}^{S}\left(\omega, \operatorname{Ext}_{R}^{i}(\omega, f)\right)$ is an epimorphism for any $0 \leqslant i \leqslant n-1$.

(3) For any exact sequence

$$
0 \rightarrow A \rightarrow B \stackrel{f}{\longrightarrow} C \rightarrow 0
$$

in $\operatorname{Mod} R$ with $A \in \Omega_{\mathcal{P}_{\omega}}^{i-1}\left(\operatorname{co} \Omega^{i+k-1}(R)\right)$, $\operatorname{Tor}_{i}^{S}\left(\omega, \operatorname{Ext}_{R}^{i}(\omega, f)\right)$ is an epimorphism for any $0 \leqslant i \leqslant n-1$.

Moreover, if $k=0$, then any of the above statements is equivalent to the following

(4) For any exact sequence

$$
0 \rightarrow A \rightarrow B \stackrel{f}{\longrightarrow} C \rightarrow 0
$$

in $\operatorname{Mod} R, \operatorname{Tor}_{i}^{S}\left(\omega, \operatorname{Ext}_{R}^{i}(\omega, f)\right)$ is an epimorphism for any $0 \leqslant i \leqslant n-1$.

Proof. (1) $\Rightarrow(2)$ Let $A=\Omega_{\mathcal{P}_{\omega}}^{i-1}\left(\cos \Omega_{\mathcal{P}_{\omega}}^{i+k-1}\left(A^{\prime}\right)\right)$ with $A^{\prime} \in \operatorname{Mod} R$. For any $i \geqslant 0$, by dimension-shifting we have an exact sequence

$$
\operatorname{Ext}_{R}^{i+k}\left(\omega, A^{\prime}\right) \stackrel{g}{\longrightarrow} \operatorname{Ext}_{R}^{i}(\omega, B) \stackrel{\operatorname{Ext}_{R}^{i}(\omega, f)}{\longrightarrow} \operatorname{Ext}_{R}^{i}(\omega, C) \rightarrow \operatorname{Ext}_{R}^{i+k+1}\left(\omega, A^{\prime}\right)
$$

in $\operatorname{Mod} S$, which induces exact sequences

$$
\operatorname{Tor}_{i}^{S}\left(\omega, \operatorname{Ext}_{R}^{i}(\omega, B)\right) \stackrel{a}{\longrightarrow} \operatorname{Tor}_{i}^{S}\left(\omega, \operatorname{Im}\left(\operatorname{Ext}_{R}^{i}(\omega, f)\right)\right) \rightarrow \operatorname{Tor}_{i-1}^{S}\left(\omega, \operatorname{Ker}\left(\operatorname{Ext}_{R}^{i}(\omega, f)\right)\right)
$$

and

$$
\operatorname{Tor}_{i}^{S}\left(\omega, \operatorname{Im}\left(\operatorname{Ext}_{R}^{i}(\omega, f)\right)\right) \stackrel{b}{\longrightarrow} \operatorname{Tor}_{i}^{S}\left(\omega, \operatorname{Ext}_{R}^{i}(\omega, C)\right) \rightarrow \operatorname{Tor}_{i}^{S}\left(\omega, \operatorname{Coker}\left(\operatorname{Ext}_{R}^{i}(\omega, f)\right)\right)
$$

in $\operatorname{Mod} R$. Since $\operatorname{Coker}\left(\operatorname{Ext}_{R}^{i}(\omega, f)\right) \subseteq \operatorname{Ext}_{R}^{i+k+1}\left(\omega, A^{\prime}\right)$, by (1) we have

$$
\operatorname{Tor}_{i}^{S}\left(\omega, \operatorname{Coker}\left(\operatorname{Ext}_{R}^{i}(\omega, f)\right)\right)=0
$$

for any $0 \leqslant i \leqslant n-1$. Moreover, it follows from (1) and the exact sequence

$$
0 \rightarrow \operatorname{Ker} g \rightarrow \operatorname{Ext}_{R}^{i+k}\left(\omega, A^{\prime}\right) \rightarrow \operatorname{Ker}\left(\operatorname{Ext}_{R}^{i}(\omega, f)\right) \rightarrow 0
$$

in $\operatorname{Mod} S$ that $\operatorname{Tor}_{i-1}^{S}\left(\omega, \operatorname{Ker}\left(\operatorname{Ext}_{R}^{i}(\omega, f)\right)\right)=0$ for any $0 \leqslant i \leqslant n-1$. Thus $\operatorname{Tor}_{i}^{S}\left(\omega, \operatorname{Ext}_{R}^{i}(\omega, f)\right)=b \cdot a$ is an epimorphism for any $0 \leqslant i \leqslant n-1$.

By Lemma $3.4(2)$, we have $(2) \Rightarrow(3)$.

$(3) \Rightarrow(1)$ Let $M \in \operatorname{Mod} R$. Fix $i(1 \leqslant i \leqslant n)$ and an $S$-submodule $L$ of $\operatorname{Ext}_{R}^{i+k}(\omega, M)$. Take an epimorphism $a: P \rightarrow L$ in $\operatorname{Mod} S$ with $P$ projective and $a^{\prime}$ the composition

$$
P \stackrel{a}{\rightarrow} L \hookrightarrow \operatorname{Ext}_{R}^{i+k}(\omega, M)
$$


Then $a^{\prime}$ can be lifted to $b: P \rightarrow \operatorname{co} \Omega^{i+k}(M)_{*}$. Take the following pull-back diagram

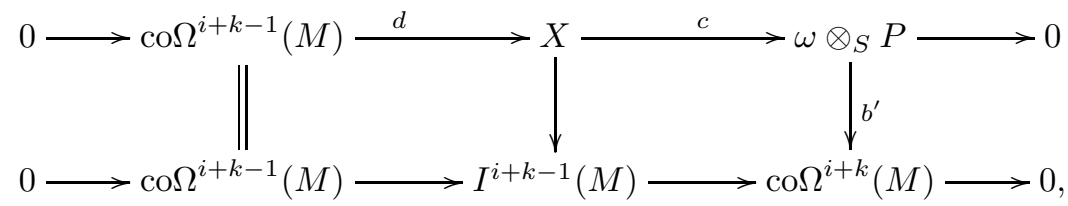

Diagram (3.3)

where $b^{\prime}$ is the composition

$$
\omega \otimes_{S} P \stackrel{1_{\omega} \otimes b}{\longrightarrow} \omega \otimes_{S} \cos \Omega^{i+k}(M)_{*} \stackrel{\theta_{\operatorname{co} \Omega^{i+k}(M)}}{\longrightarrow} \operatorname{co} \Omega^{i+k}(M) .
$$

It induces the following commutative diagram with exact rows

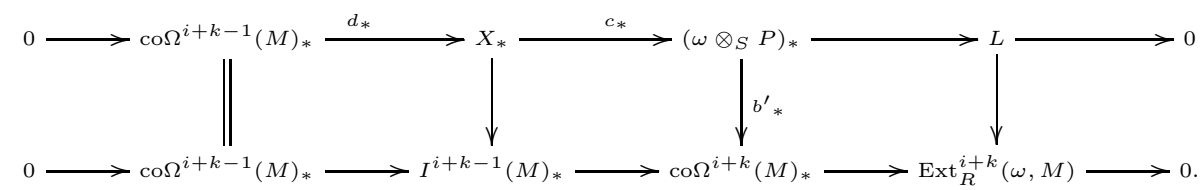

In the following, we will proceed by induction on $i$. Let $i=1$. Since $1_{\omega} \otimes c_{*}$ is epic by (3), we have that $\omega \otimes_{S} L=0$ and s.T-cograde ${ }_{\omega} \operatorname{Ext}_{R}^{1+k}(\omega, M) \geqslant 1$.

Assume that the statement (1) holds for any $1 \leqslant i \leqslant n-1$. Now consider the case for $i=n$. By the induction hypothesis, we have that s.T-cograde $\operatorname{Ext}_{R}^{i+k}(\omega, M) \geqslant i$ for any $1 \leqslant i \leqslant n-1$ and s.T-cograde ${ }_{\omega} \operatorname{Ext}_{R}^{n+k}(\omega, M) \geqslant n-1$. Then $\operatorname{co}^{n+k-1}(M) \in$ $c \mathcal{T}_{\omega}^{n-1}(R)$ by Lemma 3.2. Because $\omega \otimes_{S} P \in \mathrm{cT}_{\omega}^{n-1}(R)$ by [32, Proposition 3.7], it follows from [36, Lemma 4.3] that $X$ in the diagram (3.3) is in $\mathrm{c}^{n}{ }_{\omega}^{n-1}(R)$. By [32, Proposition 3.7] again, there exists $\operatorname{Hom}_{R}\left(\operatorname{Add}_{R} \omega,-\right)$-exact exact sequences

$$
0 \rightarrow Y^{\prime} \rightarrow W_{n-2}^{\prime} \rightarrow \cdots \rightarrow W_{0}^{\prime} \rightarrow \operatorname{co} \Omega^{n+k-1}(M) \rightarrow 0
$$

and

$$
0 \rightarrow Y \rightarrow W_{n-2} \rightarrow \cdots \rightarrow W_{0} \rightarrow X \rightarrow 0
$$

in $\operatorname{Mod} R$ with all $W_{j}^{\prime}, W_{j}$ in $\operatorname{Add}_{R} \omega$. Then both $Y$ and $Y^{\prime}$ are in $R^{\omega^{\perp_{n-1}}}$ and we get the following commutative diagram

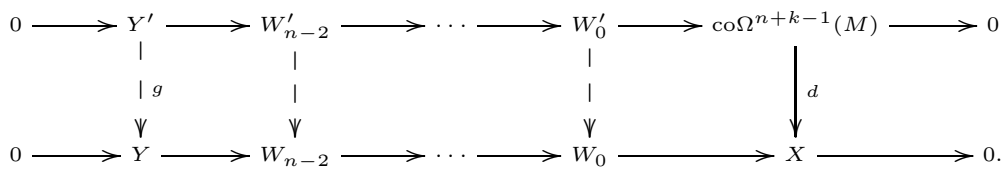

We can guarantee that $g$ is a monomorphism by adding a direct summand in $\operatorname{Add}_{R} \omega$ (for example $W_{n-2}^{\prime}$ ) to $Y$ and $W_{n-2}$. Thus we get an exact sequence

$$
0 \rightarrow Y^{\prime} \stackrel{g}{\longrightarrow} Y \stackrel{h}{\longrightarrow} Z \rightarrow 0
$$

in $\operatorname{Mod} R$ with $Z=$ Coker $g$. Since

$$
\operatorname{Coker}\left(\operatorname{Ext}_{R}^{n-1}(\omega, h)\right) \cong \operatorname{Ker}\left(\operatorname{Ext}_{R}^{n}(\omega, g)\right) \cong \operatorname{Ker}\left(\operatorname{Ext}_{R}^{1}(\omega, d)\right) \cong \operatorname{Coker} c_{*} \cong L,
$$


we obtain $L \cong \operatorname{Ext}_{R}^{n-1}(\omega, Z)$. Since $Y^{\prime} \in \Omega_{\mathcal{P}}^{n-1}\left(\operatorname{co} \Omega^{n+k-1}(R)\right)$, by $(3)$ we get that $\operatorname{Tor}_{n-1}^{S}\left(\omega, \operatorname{Ext}_{R}^{n-1}(\omega, h)\right)$ is epic. So $\operatorname{Tor}_{n-1}^{S}(\omega, L)=0$ and s.T-cograde ${ }_{\omega} \operatorname{Ext}_{R}^{n+k}(\omega, M)$ $\geqslant n$.

When $k=0$, the proof of $(3) \Rightarrow(1) \Rightarrow(2)$ is in fact that of $(4) \Leftrightarrow(1)$ by just removing the first sentence and putting $A^{\prime}=A$ in the beginning of the proof of $(1) \Rightarrow(2)$,

Compare the following result with Theorem 3.7

Theorem 3.9. For any $n \geqslant 1$ and $k \geqslant 0$, the following three statements are equivalent.

(1) s.E-cograde $\omega \operatorname{Tor}_{i+k}^{S}(\omega, N) \geqslant i$ for any $N \in \operatorname{Mod} S$ and $1 \leqslant i \leqslant n$.

(2) For any exact sequence

$$
0 \rightarrow A \stackrel{g}{\longrightarrow} B \rightarrow C \rightarrow 0
$$

in $\operatorname{Mod} S$ with $C \in \cos _{\mathcal{I}_{\omega}}^{i-1}\left(\Omega_{\mathcal{I}_{\omega}}^{i+k-1}(S)\right)$, $\operatorname{Ext}_{R}^{i}\left(\omega, \operatorname{Tor}_{i}^{S}(\omega, g)\right)$ is a monomorphism for any $0 \leqslant i \leqslant n-1$.

(3) For any exact sequence

$$
0 \rightarrow A \stackrel{g}{\longrightarrow} B \rightarrow C \rightarrow 0
$$

in $\operatorname{Mod} S$ with $C \in \operatorname{co} \Omega_{\mathcal{I}_{\omega}}^{i-1}\left(\Omega_{\mathcal{F}}^{i+k-1}(S)\right)$, $\operatorname{Ext}_{R}^{i}\left(\omega, \operatorname{Tor}_{i}^{S}(\omega, g)\right)$ is a monomorphism for any $0 \leqslant i \leqslant n-1$.

Moreover, if $k=0$, then any of the above statements is equivalent to the following

(4) For any exact sequence

$$
0 \rightarrow A \stackrel{g}{\longrightarrow} B \rightarrow C \rightarrow 0
$$

in $\operatorname{Mod} S, \operatorname{Ext}_{R}^{i}\left(\omega, \operatorname{Tor}_{i}^{S}(\omega, g)\right)$ is a monomorphism for any $0 \leqslant i \leqslant n-1$.

Proof. $(1) \Rightarrow(2)$ Let $C=\cos \Omega_{\mathcal{I}_{\omega}}^{i-1}\left(\Omega_{\mathcal{I}_{\omega}}^{i+k-1}\left(C^{\prime}\right)\right)$ with $C^{\prime} \in \operatorname{Mod} S$. For any $i \geqslant 0$, by dimension shifting we have an exact sequence

$$
\operatorname{Tor}_{i+k+1}^{S}\left(\omega, C^{\prime}\right) \rightarrow \operatorname{Tor}_{i}^{S}(\omega, A) \stackrel{\operatorname{Tor}_{i}^{S}(\omega, g)}{\longrightarrow} \operatorname{Tor}_{i}^{S}(\omega, B) \stackrel{f}{\longrightarrow} \operatorname{Tor}_{i+k}^{S}\left(\omega, C^{\prime}\right)
$$

in $\operatorname{Mod} R$, which induces exact sequences

$$
\operatorname{Ext}_{R}^{i}\left(\omega, \operatorname{Ker}\left(\operatorname{Tor}_{i}^{S}(\omega, g)\right)\right) \rightarrow \operatorname{Ext}_{R}^{i}\left(\omega, \operatorname{Tor}_{i}^{S}(\omega, A)\right) \stackrel{a}{\longrightarrow} \operatorname{Ext}_{R}^{i}\left(\omega, \operatorname{Im}\left(\operatorname{Tor}_{i}^{S}(\omega, g)\right)\right)
$$

and

$\operatorname{Ext}_{R}^{i-1}\left(\omega, \operatorname{Coker}\left(\operatorname{Tor}_{i}^{S}(\omega, g)\right)\right) \rightarrow \operatorname{Ext}_{R}^{i}\left(\omega, \operatorname{Im}\left(\operatorname{Tor}_{i}^{S}(\omega, g)\right)\right) \stackrel{b}{\longrightarrow} \operatorname{Ext}_{R}^{i}\left(\omega, \operatorname{Tor}_{i}^{S}(\omega, B)\right)$

in $\operatorname{Mod} S$. Since $\operatorname{Ker}\left(\operatorname{Tor}_{i}^{S}(\omega, g)\right)$ is an $R$-quotient module of $\operatorname{Tor}_{i+k+1}^{S}\left(\omega, C^{\prime}\right)$, by (1) we have

$$
\operatorname{Ext}_{R}^{i}\left(\omega, \operatorname{Ker}\left(\operatorname{Tor}_{i}^{S}(\omega, g)\right)\right)=0 .
$$

Moreover, it follows from (1) and the exact sequence

$$
0 \rightarrow \operatorname{Coker}\left(\operatorname{Tor}_{i}^{S}(\omega, g)\right) \rightarrow \operatorname{Tor}_{i+k}^{S}\left(\omega, C^{\prime}\right) \rightarrow \text { Coker } f \rightarrow 0
$$

in $\operatorname{Mod} R$ that $\operatorname{Ext}_{R}^{i-1}\left(\omega, \operatorname{Coker}\left(\operatorname{Tor}_{i}^{S}(\omega, g)\right)\right)=0$ for any $0 \leqslant i \leqslant n-1$. Thus $\operatorname{Ext}_{R}^{i}\left(\omega, \operatorname{Tor}_{i}^{S}(\omega, g)\right)=b \cdot a$ is a monomorphism for any $0 \leqslant i \leqslant n-1$.

By Lemma 3.6(2), we have $(2) \Rightarrow(3)$. 
(3) $\Rightarrow(1)$ Let $N \in \operatorname{Mod} S$. Fix $i(1 \leqslant i \leqslant n)$ and an $R$-quotient module $H$ of $\operatorname{Tor}_{i+k}^{S}(\omega, N)$. Take a monomorphism $a: H \longmapsto I$ in $\operatorname{Mod} R$ with $I$ injective and $a^{\prime}$ the composition

$$
\operatorname{Tor}_{i+k}^{S}(\omega, N) \rightarrow H \stackrel{a}{\rightarrow} I
$$

Then $a^{\prime}$ can be extended to $b: \omega \otimes_{S} \Omega_{\mathcal{F}}^{i+k}(N) \rightarrow I$. Take the following push-out diagram:

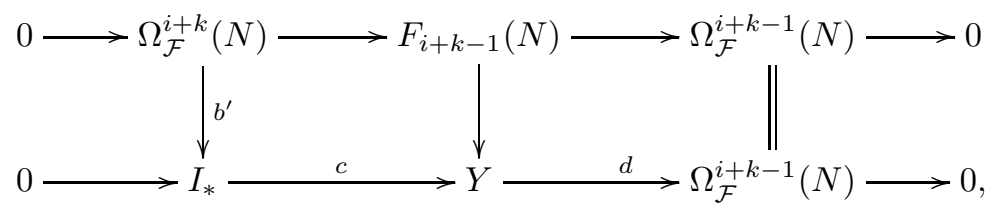

Diagram (3.5)

where $b^{\prime}$ is the composition

$$
\Omega_{\mathcal{F}}^{i+k}(N) \stackrel{\mu_{\Omega_{\mathcal{F}}^{i+k}(N)}^{\longrightarrow}}{\longrightarrow}\left(\omega \otimes_{S} \Omega_{\mathcal{F}}^{i+k}(N)\right)_{*} \stackrel{b_{*}}{\longrightarrow} I_{*} .
$$

It induces the following commutative diagram with exact rows

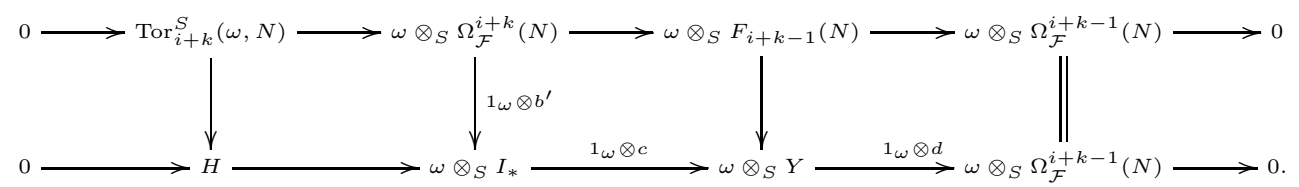

In the following, we will proceed by induction on $i$. Let $i=1$. Since $\left(1_{\omega} \otimes c\right)_{*}$ is monic by (2), we have that $H_{*}=0$ and s.E-cograde $\operatorname{Tor}_{1+k}^{S}(\omega, N) \geqslant 1$.

Assume that the statement ( 1$)$ holds for any $1 \leqslant i \leqslant n-1$. Now consider the case for $i=n$. By the induction hypothesis, we have that s.E-cograde $\operatorname{Tor}_{i+k}^{S}(\omega, N) \geqslant i$ for any $1 \leqslant i \leqslant n-1$ and s.E-cograde ${ }_{\omega} \operatorname{Tor}_{n+k}^{S}(\omega, N) \geqslant n-1$. Then $\Omega_{\mathcal{F}}^{n+k-1}(N) \in$ ac $\mathcal{T}_{\omega}^{n-1}(S)$ by Lemma 3.3. Because $I_{*} \in \operatorname{ac} \mathcal{T}_{\omega}^{n-1}(S)$ by [34, Propposition], it follows from the dual result of [36, Lemma 4.3] that $Y$ in the diagram (3.5) is in ac $\mathcal{T}_{\omega}^{n-1}(S)$. By [34, Propposition] again, there exist $\left(\omega \otimes_{S}-\right)$-exact exact sequences

$$
0 \rightarrow Y \rightarrow U^{0} \rightarrow \cdots \rightarrow U^{n-2} \rightarrow X \rightarrow 0
$$

and

$$
0 \rightarrow \Omega_{\mathcal{F}}^{n+k-1}(L) \rightarrow V^{0} \rightarrow \cdots \rightarrow V^{n-2} \rightarrow X^{\prime} \rightarrow 0
$$

in Mod $S$ with all $U^{i}, V^{i}$ in $\mathcal{I}_{\omega}(S)$. Then both $X$ and $X^{\prime}$ are in $\omega_{S}{ }^{\top_{n-1}}$ and we get the following commutative diagram

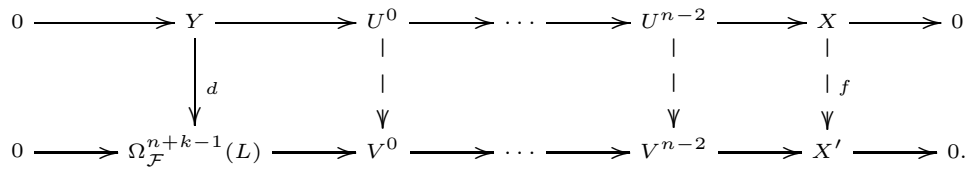

We can guarantee that $f$ is an epimorphism by adding a direct summand in $\mathcal{I}_{\omega}(S)$ (for example $V^{n-2}$ ) to $X$ and $U^{n-2}$. Thus we get an exact sequence

$$
0 \rightarrow Z \stackrel{h}{\longrightarrow} X \stackrel{f}{\longrightarrow} X^{\prime} \rightarrow 0
$$


in $\operatorname{Mod} S$ with $Z=\operatorname{Ker} f$. Since

$$
\operatorname{Ker}\left(\operatorname{Tor}_{n-1}^{S}(\omega, h)\right) \cong \operatorname{Coker}\left(\operatorname{Tor}_{n}^{S}(\omega, f)\right) \cong \operatorname{Coker}\left(\operatorname{Tor}_{1}^{S}(\omega, d)\right) \cong \operatorname{Ker}\left(1_{\omega} \otimes c\right),
$$

we obtain $H \cong \operatorname{Tor}_{n-1}^{S}(\omega, Z)$. Since $X^{\prime} \in \operatorname{co}_{\mathcal{I}_{\omega}}^{n-1}\left(\Omega_{\mathcal{F}}^{n+k-1}(S)\right)$, by (3) we get that $\operatorname{Ext}_{R}^{n-1}\left(\omega, \operatorname{Tor}_{n-1}^{S}(\omega, h)\right)$ is a monomorphism. So $\operatorname{Ext}_{R}^{n-1}(\omega, H)=0$ and s.E-cograde $\operatorname{Tor}_{n+k}^{S}(\omega, N) \geqslant n$.

When $k=0$, the proof of $(3) \Rightarrow(1) \Rightarrow(2)$ is in fact that of $(4) \Leftrightarrow(1)$ by just removing the first sentence and putting $C^{\prime}=C$ in the beginning of the proof of $(1) \Rightarrow(2)$,

\section{4. (Quasi) n-cograde condition}

In this section, we introduce and study the (quasi) $n$-cograde condition of semidualizing bimodules.

\subsection{The $n$-cograde condition}

Definition 4.1. For any $n \geqslant 1, \omega$ is said to satisfy the right $n$-cograde condition if s.E-cograde $\operatorname{Tor}_{i}^{S}(\omega, N) \geqslant i$ for any $N \in \operatorname{Mod} S$ and $1 \leqslant i \leqslant n$; and $\omega$ is said to satisfy the left $n$-cograde condition if s.E-cograde $\operatorname{Tor}_{i}^{R}\left(M^{\prime}, \omega\right) \geqslant i$ for any $M^{\prime} \in \operatorname{Mod} R^{o p}$ and $1 \leqslant i \leqslant n$.

As a consequence of Theorems 3.8 and 3.9 we get the following equivalent characterizations for $\omega$ satisfying the right $n$-cograde condition.

Corollary 4.2. For any $n \geqslant 1$, the following statements are equivalent.

(1) s.T-cograde ${ }_{\omega} \operatorname{Ext}_{R}^{i}(\omega, M) \geqslant i$ for any $M \in \operatorname{Mod} R$ and $1 \leqslant i \leqslant n$.

(2) s.E-cograde $\operatorname{Tor}_{i}^{S}(\omega, N) \geqslant i$ for any $N \in \operatorname{Mod} S$ and $1 \leqslant i \leqslant n$.

(3) $\operatorname{Tor}_{i}^{S}\left(\omega, \operatorname{Ext}_{R}^{i}(\omega,-)\right)$ preserves epimorphisms in $\operatorname{Mod} R$ for $0 \leqslant i \leqslant n-1$.

(4) $\operatorname{Ext}_{R}^{i}\left(\omega, \operatorname{Tor}_{i}^{S}(\omega,-)\right)$ preserves monomorphisms in $\operatorname{Mod} S$ for $0 \leqslant i \leqslant n-1$.

(5) For any exact sequence

$$
0 \rightarrow A \rightarrow B \stackrel{f}{\longrightarrow} C \rightarrow 0
$$

in $\operatorname{Mod} R$ with $A \in \Omega_{\mathcal{P}_{\omega}}^{i-1}\left(\cos _{\mathcal{P}_{\omega}}^{i-1}(R)\right)$, $\operatorname{Tor}_{i}^{S}\left(\omega, \operatorname{Ext}_{R}^{i}(\omega, f)\right)$ is an epimorphism for any $0 \leqslant i \leqslant n-1$.

(6) For any exact sequence

$$
0 \rightarrow A \rightarrow B \stackrel{f}{\longrightarrow} C \rightarrow 0
$$

in $\operatorname{Mod} R$ with $A \in \Omega_{\mathcal{P}_{\omega}}^{i-1}\left(\cos ^{i-1}(R)\right)$, $\operatorname{Tor}_{i}^{S}\left(\omega, \operatorname{Ext}_{R}^{i}(\omega, f)\right)$ is an epimorphism for any $0 \leqslant i \leqslant n-1$.

(7) For any exact sequence

$$
0 \rightarrow A \stackrel{g}{\longrightarrow} B \rightarrow C \rightarrow 0
$$

in $\operatorname{Mod} S$ with $C \in \operatorname{co} \Omega_{\mathcal{I}_{\omega}}^{i-1}\left(\Omega_{\mathcal{I}_{\omega}}^{i-1}(S)\right)$, $\operatorname{Ext}_{R}^{i}\left(\omega, \operatorname{Tor}_{i}^{S}(\omega, g)\right)$ is a monomorphism for any $0 \leqslant i \leqslant n-1$.

(8) For any exact sequence

$$
0 \rightarrow A \stackrel{g}{\longrightarrow} B \rightarrow C \rightarrow 0
$$

in $\operatorname{Mod} S$ with $C \in \operatorname{co} \Omega_{\mathcal{I}_{\omega}}^{i-1}\left(\Omega_{\mathcal{F}}^{i-1}(S)\right)$, $\operatorname{Ext}_{R}^{i}\left(\omega, \operatorname{Tor}_{i}^{S}(\omega, g)\right)$ is a monomorphism for any $0 \leqslant i \leqslant n-1$. 
Proof. By [33, Theorem 6.9], we have $(1) \Leftrightarrow(2)$. By Theorems 3.8 and 3.9 we have $(1) \Leftrightarrow(3) \Leftrightarrow(5) \Leftrightarrow(6)$ and $(2) \Leftrightarrow(4) \Leftrightarrow(7) \Leftrightarrow(8)$ respectively.

Symmetrically, we have the following equivalent characterizations for $\omega$ satisfying the left $n$-cograde condition.

Corollary 4.3. For any $n \geqslant 1$, the following statements are equivalent.

(1) s.T-cograde $\operatorname{Ext}_{S o p}^{i}\left(\omega, N^{\prime}\right) \geqslant i$ for any $N^{\prime} \in \operatorname{Mod} S^{o p}$ and $1 \leqslant i \leqslant n$.

(2) s.E-cograde $\operatorname{Tor}_{i}^{R}\left(M^{\prime}, \omega\right) \geqslant i$ for any $M^{\prime} \in \operatorname{Mod} R^{\text {op }}$ and $1 \leqslant i \leqslant n$.

(3) $\operatorname{Tor}_{i}^{R}\left(\operatorname{Ext}_{S o p}^{i}(\omega,-), \omega\right)$ preserves epimorphisms in $\operatorname{Mod} S^{o p}$ for $0 \leqslant i \leqslant$ $n-1$.

(4) $\operatorname{Ext}_{S o p}^{i}\left(\omega, \operatorname{Tor}_{i}^{R}(-, \omega)\right)$ preserves monomorphisms in $\operatorname{Mod} R^{o p}$ for $0 \leqslant i \leqslant$ $n-1$.

(5) For any exact sequence

$$
0 \rightarrow A \rightarrow B \stackrel{f}{\longrightarrow} C \rightarrow 0
$$

in $\operatorname{Mod} S^{o p}$ with $A \in \Omega_{\mathcal{P}_{\omega}}^{i-1}\left(\cos \Omega_{\mathcal{P}_{\omega}}^{i-1}\left(S^{o p}\right)\right)$, $\operatorname{Tor}_{i}^{R}\left(\operatorname{Ext}_{S^{o p}}^{i}(\omega, f), \omega\right)$ is an epimorphism for any $0 \leqslant i \leqslant n-1$.

(6) For any exact sequence

$$
0 \rightarrow A \rightarrow B \stackrel{f}{\longrightarrow} C \rightarrow 0
$$

in Mod $S^{o p}$ with $A \in \Omega_{\mathcal{P}_{\omega}}^{i-1}\left(\operatorname{co} \Omega^{i-1}\left(S^{o p}\right)\right)$, $\operatorname{Tor}_{i}^{R}\left(\operatorname{Ext}_{S^{o p}}^{i}(\omega, f), \omega\right)$ is an epimorphism for any $0 \leqslant i \leqslant n-1$.

(7) For any exact sequence

$$
0 \rightarrow A \stackrel{g}{\longrightarrow} B \rightarrow C \rightarrow 0
$$

in $\operatorname{Mod} R^{o p}$ with $C \in \operatorname{co} \Omega_{\mathcal{I}_{\omega}}^{i-1}\left(\Omega_{\mathcal{I}_{\omega}}^{i-1}\left(R^{o p}\right)\right)$, $\operatorname{Ext}_{S o p}^{i}\left(\omega, \operatorname{Tor}_{i}^{R}(g, \omega)\right)$ is a monomorphism for any $0 \leqslant i \leqslant n-1$.

(8) For any exact sequence

$$
0 \rightarrow A \stackrel{g}{\longrightarrow} B \rightarrow C \rightarrow 0
$$

in $\operatorname{Mod} R^{o p}$ with $C \in \cos \Omega_{\mathcal{I}_{\omega}}^{i-1}\left(\Omega_{\mathcal{F}}^{i-1}\left(R^{o p}\right)\right)$, $\operatorname{Ext}_{S^{o p}}^{i}\left(\omega, \operatorname{Tor}_{i}^{R}(g, \omega)\right)$ is a monomorphism for any $0 \leqslant i \leqslant n-1$.

In the following, we will establish the left-right symmetry of the $n$-cograde condition.

Lemma 4.4. Let

$$
0 \rightarrow A \rightarrow B \rightarrow C \rightarrow 0
$$

be an exact sequence in $\operatorname{Mod} R$ such that $A$ is superfluous in $B$. Then the following assertions hold.

(1) Let $L \in \operatorname{Mod} R^{o p}$. If $L^{\prime} \otimes_{R} C=0$ for any submodule $L^{\prime}$ of $L$, then $L \otimes_{R} B=$ 0 .

(2) Let $M \in \operatorname{Mod} R$. If $\operatorname{Hom}_{R}\left(C, M^{\prime}\right)=0$ for any quotient module $M^{\prime}$ of $M$, then $\operatorname{Hom}_{R}(B, M)=0$.

Proof. (1) If $L \otimes_{R} B \neq 0$, then there exists $x \in L$ such that $x R \otimes_{R} B \neq 0$. Since $x R \cong R / I$ for some right ideal $I$ of $R$, we have that

$$
B / I B \cong R / I \otimes_{R} B \cong x R \otimes_{R} B \neq 0
$$


and $I B \supsetneqq B$. In view of the assumption that $A$ is superfluous in $B$, it follows that $I B+A \supsetneqq B$ and

$$
x R \otimes_{R} C \cong R / I \otimes_{R} C \cong R / I \otimes_{R} B / A \cong \frac{B / A}{(I B+A) / A} \cong B /(I B+A) \neq 0 .
$$

It contradicts the assumption.

(2) If $\operatorname{Hom}_{R}(B, M) \neq 0$, then there exists a non-zero homomorphism $f \in$ $\operatorname{Hom}_{R}(B, M)$. Pick the kernel $L$ of $f$ such that $\operatorname{Im} f \cong B / L$. Because $A$ is superfluous in $B$ and $f \neq 0$, we have $A+L \supsetneqq B$. Then there exists a non-zero natural epimorphism $\pi: B / A(\cong C) \rightarrow B /(A+L)$. Note that the inclusions $(A+L) / L \subseteq B / L \subseteq M$ induce an embedding homomorphism

$$
i: \frac{B / L}{(A+L) / L}(\cong B /(A+L)) \hookrightarrow \frac{M}{(A+L) / L} .
$$

Then $0 \neq i \cdot \pi \in \operatorname{Hom}_{R}\left(C, \frac{M}{(A+L) / L}\right)$, which contradicts the assumption.

It is straightforward to verify the following observation.

\section{Lemma 4.5.}

(1) If $P \in \operatorname{Mod} R$ is finitely generated projective, then $\operatorname{pd}_{S^{o p}} P^{*}=\mathcal{P}_{\omega}(R)$ $\operatorname{id}_{R} P$.

(2) If $Q \in \operatorname{Mod} S^{o p}$ is finitely generated projective, then $\operatorname{pd}_{R} Q^{*}=\mathcal{P}_{\omega}\left(S^{o p}\right)$ $\operatorname{id}_{S^{o p}} Q$.

Lemma 4.6. Let $P \in \operatorname{Mod} R$ be finitely generated projective and $t \geqslant 0$. Then the following statements are equivalent.

(1) $\operatorname{pd}_{S^{o p}} P^{*} \leqslant t$.

(2) $\mathcal{P}_{\omega}(R)-\operatorname{id}_{R} P \leqslant t$.

(3) $\operatorname{Ext}_{S^{o p}}^{t+1}(\omega, H) \otimes_{R} P=0$ for any $H \in \operatorname{Mod} S^{o p}$.

(4) $\operatorname{Hom}_{R}\left(P, \operatorname{Tor}_{t+1}^{S}(\omega, N)\right)=0$ for any $N \in \operatorname{Mod} S$.

Proof. By Lemma 4.5(1), we have $(1) \Leftrightarrow(2)$.

(1) $\Leftrightarrow(3)$ Let $H \in \operatorname{Mod} S^{o p}$ and

$$
\mathbf{I}:=0 \rightarrow H \rightarrow I^{0} \rightarrow I^{1} \rightarrow \cdots \rightarrow I^{i} \rightarrow \cdots
$$

be an injective resolution of $H$ in $\operatorname{Mod} S^{o p}$. Because $P \in \operatorname{Mod} R$ is finitely generated projective by assumption, the functor $-\otimes_{R} P$ is exact. Then we have

$$
\begin{aligned}
& \operatorname{Ext}_{S^{o p}}^{t+1}\left(P^{*}, H\right) \\
\cong & H^{t+1}\left(\operatorname{Hom}_{S^{o p}}\left(P^{*}, \mathbf{I}\right)\right) \\
\cong & H^{t+1}\left(\operatorname{Hom}_{S^{o p}}(\omega, \mathbf{I}) \otimes_{R} P\right) \\
\cong & H^{t+1}\left(\operatorname{Hom}_{S^{o p}}(\omega, \mathbf{I})\right) \otimes_{R} P(\text { by }[6, \text { p.33, Excercise 3] }) \\
\cong & \operatorname{Ext}_{S^{o p}}^{t+1}(\omega, H) \otimes_{R} P .
\end{aligned}
$$

Now the assertion follows easily. 7.6].

(1) $\Leftrightarrow(4)$ Since $\operatorname{pd}_{S^{o p}} P^{*}=\operatorname{fd}_{S^{o p}} P^{*}$, the assertion follows from [35, Lemma

Recall from [30] that a ring $R$ is called semiregular if $R / J(R)$ is von Neumann regular and idempotents can be lifted modulo $J(R)$, where $J(R)$ is the Jacobson radical of $R$. The class of semiregular rings includes: (1) von Neumann regular 
rings; (2) semiperfect rings; (3) left cotorsion rings; and (4) right cotorsion rings. See [18] for the definitions of left cotorsion rings and right cotorsion rings.

If $R$ is a semiregular ring, then any finitely presented left or right $R$-module has a projective cover by [30, Theorem 2.9]. In this case, since ${ }_{R} \omega$ admits a degreewise finite $R$-projective resolution by Definition 2.1, we may assume that

$$
\cdots \rightarrow P_{i}(\omega) \rightarrow \cdots \rightarrow P_{1}(\omega) \rightarrow P_{0}(\omega) \rightarrow{ }_{R} \omega \rightarrow 0
$$

is the minimal projective resolution of ${ }_{R} \omega$ in $\bmod R$. Put $\omega_{i}:=\operatorname{Im}\left(P_{i}(\omega) \rightarrow\right.$ $\left.P_{i-1}(\omega)\right)$ for any $i \geqslant 1$ and $\omega_{0}:=\omega$. Analogously, if $S$ is a semiregular ring, then we assume that

$$
\cdots \rightarrow Q_{i}(\omega) \rightarrow \cdots \rightarrow Q_{1}(\omega) \rightarrow Q_{0}(\omega) \rightarrow \omega_{S} \rightarrow 0
$$

is the minimal projective resolution of $\omega_{S}$ in $\bmod S^{o p}$. By Lemma 4.6, we have the following

Proposition 4.7. Let $R$ be a semiregular ring and $m, n \geqslant 1$. Then the following statements are equivalent.

(1) $\operatorname{pd}_{S^{o p}} P_{i}(\omega)^{*} \leqslant m-1$ for any $0 \leqslant i \leqslant n-1$.

(2) $\mathcal{P}_{\omega}(R)-\operatorname{id}_{R} P_{i}(\omega) \leqslant m-1$ for any $0 \leqslant i \leqslant n-1$.

(3) s.T-cograde $\omega \operatorname{Ext}_{S o p}^{m}\left(\omega, N^{\prime}\right) \geqslant n$ for any $N^{\prime} \in \operatorname{Mod} S^{o p}$.

(4) s.E-cograde $\operatorname{Tor}_{m}^{S}(\omega, N) \geqslant n$ for any $N \in \operatorname{Mod} S$.

Proof. By [35, Proposition 7.7] and Lemma 4.6, we have (4) $\Leftrightarrow(1) \Leftrightarrow(2)$.

$(3) \Rightarrow(1)$ We proceed by induction on $n$. Let $N^{\prime} \in \operatorname{Mod} S^{o p}$. Suppose $n=1$. Because s.T-cograde ${ }_{\omega} \operatorname{Ext}_{S^{o p}}^{m}\left(\omega, N^{\prime}\right) \geqslant 1$ by (3), we have $L^{\prime} \otimes_{R} \omega=0$ for any submodule $L^{\prime}$ of $\operatorname{Ext}_{S^{o p}}^{m}\left(\omega, N^{\prime}\right)$ in Mod $R^{o p}$. It follows from Lemma 4.4(1) that $\operatorname{Ext}_{S^{o p}}^{m}\left(\omega, N^{\prime}\right) \otimes_{R} P_{0}(\omega)=0$. Therefore by Lemma 4.6 we get $\operatorname{pd}_{S^{o p}} P_{0}(\omega)^{*} \leqslant m-1$ and the case for $n=1$ is proved.

Now suppose $n \geqslant 2$. Let $X$ be a submodule of $\operatorname{Ext}_{S^{o p}}^{m}\left(\omega, N^{\prime}\right)$ in $\operatorname{Mod} R^{o p}$. By (3), we have $\operatorname{Tor}_{0 \leqslant i \leqslant n-1}^{R}(X, \omega)=0$. Then for any $0 \leqslant i \leqslant n-2$, we have

$$
\operatorname{Tor}_{1}^{R}\left(X, \omega_{i}\right) \cong \operatorname{Tor}_{i+1}^{R}(X, \omega)=0 .
$$

For any $i \geqslant 0$, from the exact sequence

$$
0 \rightarrow \omega_{i+1} \rightarrow P_{i}(\omega) \rightarrow \omega_{i} \rightarrow 0,
$$

we get the following exact sequence

$$
0 \rightarrow \operatorname{Tor}_{1}^{R}\left(X, \omega_{i}\right) \rightarrow X \otimes_{R} \omega_{i+1} \rightarrow X \otimes_{R} P_{i}(\omega) .
$$

By the induction hypothesis, we have $\operatorname{pd}_{S o p} P_{i}(\omega)^{*} \leqslant m-1$ for any $0 \leqslant i \leqslant n-2$. Then it follows from Lemma 4.6 that $\operatorname{Ext}_{S^{o p}}^{m}\left(\omega, N^{\prime}\right) \otimes_{R} P_{n-2}(\omega)=0$ and hence $X \otimes_{R} P_{n-2}(\omega)=0$. So it is derived from (4.1) that $X \otimes_{R} \omega_{n-1}=0$. Notice that $P_{n-1}(\omega)$ is the projective cover of $\omega_{n-1}$, so $\operatorname{Ext}_{S^{o p}}^{m}\left(\omega, N^{\prime}\right) \otimes_{R} P_{n-1}(\omega)=0$ by Lemma 4.4(1). It follows from Lemma 4.6 that $\operatorname{pd}_{S^{o p}} P_{n-1}(\omega)^{*} \leqslant m-1$.

$(1) \Rightarrow(3)$ Let $X$ be a submodule of $\operatorname{Ext}_{S^{o p}}^{m}\left(\omega, N^{\prime}\right)$ in $\operatorname{Mod} R^{o p}$. Then by (1) and Lemma 4.6, we have $\operatorname{Ext}_{S^{o p}}^{m}\left(\omega, N^{\prime}\right) \otimes_{R}\left(\oplus_{i=0}^{n-1} P_{i}(\omega)\right)=0$, and hence $X \otimes_{R}$ $\left(\oplus_{i=0}^{n-1} P_{i}(\omega)\right)=0$. Since $\omega_{i}$ is a quotient module of $P_{i}(\omega)$ for any $i \geqslant 0$, we then have $X \otimes_{R}\left(\oplus_{i=0}^{n-1} \omega_{i}\right)=0$.

If $n=1$, then $X \otimes_{R} \omega=0$ and s.T-cograde ${ }_{\omega} \operatorname{Ext}_{S^{o p}}^{m}\left(\omega, N^{\prime}\right) \geqslant 1$. If $n \geqslant 2$, then from (4.1) we get $\operatorname{Tor}_{1}^{R}\left(X, \oplus_{i=0}^{n-2} \omega_{i}\right)=0$. Since $\operatorname{Tor}_{i+1}^{R}(X, \omega) \cong \operatorname{Tor}_{1}^{R}\left(X, \omega_{i}\right)$ for any $i \geqslant 0$, we have that $\operatorname{Tor}_{0 \leqslant i \leqslant n-1}^{R}(X, \omega)=0$ and s.T-cograde $\operatorname{Ext}_{S^{o p}}^{m}\left(\omega, N^{\prime}\right) \geqslant n$. 
The following result means that the $n$-cograde condition is left-right symmetric.

Theorem 4.8. Let $R$ be semiregular and $n \geqslant 1$. Then the following statements are equivalent.

(1) $\operatorname{pd}_{S_{o p}} P_{i}(\omega)^{*} \leqslant i$ for any $0 \leqslant i \leqslant n-1$.

(2) $\mathcal{P}_{\omega}(R)-\mathrm{id}_{R} P_{i}(\omega) \leqslant i$ for any $0 \leqslant i \leqslant n-1$.

(3) s.T-cograde $\operatorname{Ext}_{R}^{i}(\omega, M) \geqslant i$ for any $M \in \operatorname{Mod} R$ and $1 \leqslant i \leqslant n$.

(4) s.E-cograde $\operatorname{Tor}_{i}^{S}(\omega, N) \geqslant i$ for any $N \in \operatorname{Mod} S$ and $1 \leqslant i \leqslant n$.

(5) s.T-cograde $\operatorname{Ext}_{S^{o p}}^{i}\left(\omega, N^{\prime}\right) \geqslant i$ for any $N^{\prime} \in \operatorname{Mod} S^{o p}$ and $1 \leqslant i \leqslant n$.

(6) $\mathrm{s} . \mathrm{E}-\operatorname{cograde}{ }_{\omega} \operatorname{Tor}_{i}^{R}\left(M^{\prime}, \omega\right) \geqslant i$ for any $M^{\prime} \in \operatorname{Mod} R^{\text {op }}$ and $1 \leqslant i \leqslant n$.

(7) $\operatorname{Tor}_{i}^{S}\left(\omega, \operatorname{Ext}_{R}^{i}(\omega,-)\right)$ preserves epimorphisms in $\operatorname{Mod} R$ for $0 \leqslant i \leqslant n-1$.

(8) $\operatorname{Ext}_{R}^{i}\left(\omega, \operatorname{Tor}_{i}^{S}(\omega,-)\right)$ preserves monomorphisms in $\operatorname{Mod} S$ for $0 \leqslant i \leqslant n-1$.

(9) $\operatorname{Tor}_{i}^{R}\left(\operatorname{Ext}_{S^{o p}}^{i}(\omega,-), \omega\right)$ preserves epimorphisms in $\operatorname{Mod} S^{o p}$ for $0 \leqslant i \leqslant$ $n-1$.

(10) $\operatorname{Ext}_{S^{\circ o}}^{i}\left(\omega, \operatorname{Tor}_{i}^{R}(-, \omega)\right)$ preserves monomorphisms in $\operatorname{Mod} R^{\text {op }}$ for $0 \leqslant i \leqslant$ $n-1$.

Proof. By Proposition 4.7 we have (1) $\Leftrightarrow(2) \Leftrightarrow(4) \Leftrightarrow$ (5). By Corollaries 4.2 and 4.3 , we have $(3) \Leftrightarrow(4) \Leftrightarrow(7) \Leftrightarrow(8)$ and $(5) \Leftrightarrow(6) \Leftrightarrow(9) \Leftrightarrow(10)$.

As a consequence, we get the following

Corollary 4.9. Let $R$ and $S$ be semiregular and $n \geqslant 1$. Then the following statements are equivalent.

(1) $\operatorname{pd}_{S^{o p}} P_{i}(\omega)^{*} \leqslant i$ for any $0 \leqslant i \leqslant n-1$.

(2) $\operatorname{pd}_{R} Q_{i}(\omega)^{*} \leqslant i$ for any $0 \leqslant i \leqslant n-1$.

(3) $\mathcal{P}_{\omega}(R)-\operatorname{id}_{R} P_{i}(\omega) \leqslant i$ for any $0 \leqslant i \leqslant n-1$.

(4) $\mathcal{P}_{\omega}\left(S^{o p}\right)-\operatorname{id}_{S^{o p}} Q_{i}(\omega) \leqslant i$ for any $0 \leqslant i \leqslant n-1$.

Proof. By the symmetric version of Proposition 4.7, we have

$(2) \Leftrightarrow(4) \Leftrightarrow \mathrm{s} . \mathrm{T}-\operatorname{cograde}_{\omega} \operatorname{Ext}_{R}^{i}(\omega, M) \geqslant i$ for any $M \in \operatorname{Mod} R$ and $1 \leqslant i \leqslant n$.

Now the assertion follows from Theorem 4.8 .

\subsection{The quasi $n$-cograde condition}

Definition 4.10. For any $n \geqslant 1, \omega$ is said to satisfy the right quasi $n$-cograde condition if s.E-cograde $\operatorname{Tor}_{i+1}^{S}(\omega, N) \geqslant i$ for any $N \in \operatorname{Mod} S$ and $1 \leqslant i \leqslant n$; and $\omega$ is said to satisfy the left quasi $n$-cograde condition if s.E-cograde $\omega_{\omega} \operatorname{Tor}_{i+1}^{R}\left(M^{\prime}, \omega\right) \geqslant i$ for any $M^{\prime} \in \operatorname{Mod} R^{o p}$ and $1 \leqslant i \leqslant n$.

It is trivial that $\omega$ satisfies the right (resp. left) quasi $n$-cograde conditions if it satisfies the right (resp. left) $n$-cograde condition. But the converse does not hold true in general, see Subsection 4.4 below.

The following lemma is useful in the sequel.

Lemma 4.11. For any $n \geqslant 0$, the following assertions hold.

(1) Let $M \in \operatorname{Mod} R$. If E-cograde $\omega \geqslant n$ and $\mathrm{T}-\operatorname{cograde}_{\omega} \operatorname{Ext}_{R}^{n}(\omega, M) \geqslant$ $n+1$, then E-cograde $\omega \geqslant n+1$.

(2) Let $N \in \operatorname{Mod} S$. If T-cograde $\omega \geqslant n$ and ${\mathrm{E}-\operatorname{cograde}_{\omega}} \operatorname{Tor}_{n}^{S}(\omega, N) \geqslant n+1$, then T-cograde $\omega \geqslant n+1$. 
Proof. We proceed by induction on $n$.

(1) If $n=0$, then $\omega \otimes_{S} M_{*}=0$ by assumption. It follows from [33, Lemma 6.1(1)] that $M_{*}=0$ and E-cograde $\omega \geqslant 1$.

Let $n \geqslant 1$. Consider an injective resolution

$$
0 \rightarrow M \rightarrow I^{0} \rightarrow \cdots \rightarrow I^{n} \rightarrow \cdots
$$

of $M$ in $\operatorname{Mod} R$. Put $M^{\prime}=\operatorname{Im}\left(I^{n-1} \rightarrow I^{n}\right)$. Since E-cograde ${ }_{\omega} M \geqslant n$ by the induction hypothesis, applying the functor $(-)_{*}$ to the above exact sequence yields the following exact sequence

$$
0 \rightarrow I^{0}{ }_{*} \rightarrow \cdots \rightarrow I^{n-1}{ }_{*} \stackrel{g}{\longrightarrow} M^{\prime}{ }_{*} \rightarrow \operatorname{Ext}_{R}^{n}(\omega, M) \rightarrow 0
$$

in Mod $S$. Because T-cograde $\operatorname{Ext}_{R}^{n}(\omega, M) \geqslant n+1$ by assumption, we have $\operatorname{Tor}_{0 \leqslant i \leqslant n}^{S}\left(\omega, \operatorname{Ext}_{R}^{n}(\omega, M)\right)=0$. Then by [11, Proposition VI.5.1], we have

$$
\operatorname{Ext}_{S}^{i}\left(\operatorname{Ext}_{R}^{n}(\omega, M), I^{j}{ }_{*}\right) \cong \operatorname{Hom}_{R}\left(\operatorname{Tor}_{i}^{S}\left(\omega, \operatorname{Ext}_{R}^{n}(\omega, M)\right), I^{j}\right)=0
$$

for any $0 \leqslant i \leqslant n$ and $j \geqslant 0$, and hence

$$
\operatorname{Ext}_{S}^{1}\left(\operatorname{Ext}_{R}^{n}(\omega, M), \operatorname{Im} g\right) \cong \operatorname{Ext}_{S}^{n}\left(\operatorname{Ext}_{R}^{n}(\omega, M), I^{0}{ }_{*}\right)=0 .
$$

It implies that the exact sequence

$$
0 \rightarrow \operatorname{Im} g \rightarrow M^{\prime}{ }_{*} \rightarrow \operatorname{Ext}_{R}^{n}(\omega, M) \rightarrow 0
$$

splits and hence $\operatorname{Ext}_{R}^{n}(\omega, M)$ is a direct summand of $M^{\prime}{ }_{*}$. Since $M^{\prime}{ }_{*}$ is adjoint 1- $\omega$-cotorsionfree, so is $\operatorname{Ext}_{R}^{n}(\omega, M)$. Thus, applying [34, Proposition 3.2], the Tcograde condition on $\operatorname{Ext}_{R}^{n}(\omega, M)$ proves $\operatorname{Ext}_{R}^{n}(\omega, M)=0$. Consequently we have E-cograde $\omega \geqslant n+1$ and the assertion follows.

(2) If $n=0$, then $\left(\omega \otimes_{S} N\right)_{*}=0$ by assumption. It follows from [33, Lemma $6.1(2)]$ that $\omega \otimes_{S} N=0$ and T-cograde $\omega$

Let $n \geqslant 1$. Consider a projective resolution

$$
\cdots \rightarrow P_{n} \rightarrow \cdots \rightarrow P_{0} \rightarrow N \rightarrow 0
$$

of $N$ in $\operatorname{Mod} S$. Put $N^{\prime}=\operatorname{Im}\left(P_{n} \rightarrow P_{n-1}\right)$. Since T-cograde $\omega_{\omega} \geqslant n$ by the induction hypothesis, applying the functor $\omega \otimes_{S}-$ to the above exact sequence yields the following exact sequence

$$
0 \rightarrow \operatorname{Tor}_{n}^{S}(\omega, N) \rightarrow \omega \otimes_{S} N^{\prime} \stackrel{f}{\longrightarrow} \omega \otimes_{S} P_{n-1} \rightarrow \cdots \rightarrow \omega \otimes_{S} P_{0} \rightarrow 0
$$

in $\operatorname{Mod} R$. Because E-cograde $\operatorname{Tor}_{n}^{S}(\omega, N) \geqslant n+1$ by assumption, we have $\operatorname{Ext}_{R}^{0 \leqslant i \leqslant n}\left(\omega, \operatorname{Tor}_{n}^{S}(\omega, N)\right)=0$. Notice that $\omega \otimes_{S} P \in \operatorname{Add}_{R} \omega$ for any projective module $P$ in $\operatorname{Mod} S$, so $\operatorname{Ext}_{R}^{0 \leqslant i \leqslant n}\left(\omega \otimes_{S} P_{j}\right.$, $\left.\operatorname{Tor}_{n}^{S}(\omega, N)\right)=0$ for any $j \geqslant 0$, and hence

$$
\operatorname{Ext}_{R}^{1}\left(\operatorname{Im} f, \operatorname{Tor}_{n}^{S}(\omega, N)\right) \cong \operatorname{Ext}_{R}^{n}\left(\omega \otimes_{S} P_{0}, \operatorname{Tor}_{n}^{S}(\omega, N)\right)=0
$$

It induces an exact sequence

$$
\operatorname{Hom}_{R}\left(\omega \otimes_{S} N^{\prime}, \operatorname{Tor}_{n}^{S}(\omega, N)\right) \rightarrow \operatorname{Hom}_{R}\left(\operatorname{Tor}_{n}^{S}(\omega, N), \operatorname{Tor}_{n}^{S}(\omega, N)\right) \rightarrow 0 .
$$

Because $\omega \otimes_{S} N^{\prime} \in \mathrm{c} \mathcal{T}_{\omega}^{1}(R)$ by [33, Lemma 6.1(2)], there exists an epimorphism $U \rightarrow \omega \otimes_{S} N^{\prime}$ in $\operatorname{Mod} R$ with $U \in \operatorname{Add}_{R} \omega$ by [32, Lemma 3.6(1)]. Because $\left(\operatorname{Tor}_{n}^{S}(\omega, N)\right)_{*}=0$, we have $\operatorname{Hom}_{R}\left(U, \operatorname{Tor}_{n}^{S}(\omega, N)\right)=0$. It follows that $\operatorname{Hom}_{R}\left(\omega \otimes_{S}\right.$ $\left.N^{\prime}, \operatorname{Tor}_{n}^{S}(\omega, N)\right)=0$ and $\operatorname{Hom}_{R}\left(\operatorname{Tor}_{n}^{S}(\omega, N), \operatorname{Tor}_{n}^{S}(\omega, N)\right)=0$, which implies $\operatorname{Tor}_{n}^{S}(\omega, N)=$ 0 . So T-cograde $\omega \geqslant n+1$ and the assertion follows. 
We have the following equivalent characterizations for $\omega$ satisfying the right quasi $n$-cograde condition.

Proposition 4.12. For any $n \geqslant 1$, the following statements are equivalent.

(1) s.E-cograde $\operatorname{Tor}_{i+1}^{S}(\omega, N) \geqslant i$ for any $N \in \operatorname{Mod} S$ and $1 \leqslant i \leqslant n$.

(2) T-cograde $\operatorname{Ext}_{R}^{i}(\omega, M) \geqslant i$ for any $M \in \operatorname{Mod} R$ and $1 \leqslant i \leqslant n$.

(3) For any exact sequence

$$
0 \rightarrow A \stackrel{g}{\longrightarrow} B \rightarrow C \rightarrow 0
$$

in $\operatorname{Mod} S$ with $C \in \operatorname{co} \Omega_{\mathcal{I}_{\omega}}^{i-1}\left(\Omega_{\mathcal{I}_{\omega}}^{i}(S)\right), \operatorname{Ext}_{R}^{i}\left(\omega, \operatorname{Tor}_{i}^{S}(\omega, g)\right)$ is a monomorphism for any $0 \leqslant i \leqslant n-1$.

(4) For any exact sequence

$$
0 \rightarrow A \stackrel{g}{\longrightarrow} B \rightarrow C \rightarrow 0
$$

in $\operatorname{Mod} S$ with $C \in \cos \Omega_{\mathcal{I}_{\omega}}^{i-1}\left(\Omega_{\mathcal{F}}^{i}(S)\right)$, $\operatorname{Ext}_{R}^{i}\left(\omega, \operatorname{Tor}_{i}^{S}(\omega, g)\right)$ is a monomorphism for any $0 \leqslant i \leqslant n-1$.

(5) $\operatorname{Tor}_{i}^{S}\left(\omega, \operatorname{Ext}_{R}^{i}(\omega, f)\right)$ is an epimorphism for any epimorphism $f: B \rightarrow C$ in $\operatorname{Mod} R$ with $B, C \in \cos \Omega_{\mathcal{P}_{\omega}}^{1}(R)$ and $0 \leqslant i \leqslant n-1$.

(6) $\operatorname{Tor}_{i}^{S}\left(\omega, \operatorname{Ext}_{R}^{i}(\omega, f)\right)$ is an epimorphism for any epimorphism $f: B \rightarrow C$ in $\operatorname{Mod} R$ with $B, C \in \operatorname{co} \Omega^{1}(R)$ and $0 \leqslant i \leqslant n-1$.

(7) $\cos \Omega^{i}(R) \subseteq \mathrm{c} \mathcal{T}_{\omega}^{i+1}(R)$ for any $1 \leqslant i \leqslant n$.

Proof. By Theorems 3.9 and 3.5, we have (1) $\Leftrightarrow(3) \Leftrightarrow(4)$ and $(2) \Leftrightarrow(5) \Leftrightarrow(6) \Leftrightarrow$ (7) respectively. In the following, we will prove $(1) \Leftrightarrow(2)$ by induction on $n$.

(1) $\Rightarrow(2)$ Let $M \in \operatorname{Mod} R$. By Lemma 3.1(1), for any $n \geqslant 1$, there exist exact sequences

$$
\begin{gathered}
0 \rightarrow \operatorname{Ext}_{R}^{n}(\omega, M) \stackrel{\lambda}{\longrightarrow} \operatorname{cTr}_{\omega} \operatorname{co} \Omega^{n-1}(M) \stackrel{\pi}{\longrightarrow} C \rightarrow 0, \\
0 \rightarrow C \rightarrow I^{n+1}(M)_{*} \rightarrow \operatorname{cTr}_{\omega} \cos \Omega^{n}(M) \rightarrow 0
\end{gathered}
$$

in Mod $S$ such that $1_{\omega} \otimes \pi$ is an isomorphism, where $C=I^{n}(M)_{*} / \cos \Omega^{n}(M)_{*}$. Because $I^{n+1}(M)_{*} \in \omega_{S}^{\top}$ by [19, Corollary 6.1], it follows from the exact sequence (4.3) that $\operatorname{Tor}_{i}^{S}(\omega, C) \cong \operatorname{Tor}_{i+1}^{S}\left(\omega, c \operatorname{Tr}_{\omega} \cos \Omega^{n}(M)\right)$ for any $i \geqslant 1$.

If $n=1$, then from the exact sequence (4.2) we get an exact sequence

$$
\operatorname{Tor}_{2}^{S}\left(\omega, \operatorname{cTr}_{\omega} \operatorname{co} \Omega^{1}(M)\right)\left(\cong \operatorname{Tor}_{1}^{S}(\omega, C)\right) \rightarrow \omega \otimes_{S} \operatorname{Ext}_{R}^{1}(\omega, M) \rightarrow 0
$$

in $\operatorname{Mod} R$. Because s.E-cograde $\operatorname{Tor}_{2}^{S}\left(\omega, c \operatorname{Tr}_{\omega} \operatorname{co} \Omega^{1}(M)\right) \geqslant 1$ by assumption, we have E-cograde $\left.\omega \otimes_{S} \operatorname{Ext}_{R}^{1}(\omega, M)\right) \geqslant 1$. It is derived from Lemma 4.11(2) that T-cograde ${ }_{\omega} \operatorname{Ext}_{R}^{1}(\omega, M) \geqslant 1$.

Now suppose $n \geqslant 2$. Then T-cograde $\operatorname{Ext}_{R}^{i}(\omega, M) \geqslant i$ for any $1 \leqslant i \leqslant n-$ 1 and T-cograde ${ }_{\omega} \operatorname{Ext}_{R}^{n}(\omega, M) \geqslant n-1$ by the induction hypothesis. It follows from Theorem 3.5 that $\operatorname{co} \Omega^{i}(R) \subseteq \mathrm{c} \mathcal{T}_{\omega}^{i}(R)$ for any $1 \leqslant i \leqslant n$. So $\operatorname{co} \Omega^{n-1}(M) \in$ $c \mathcal{T}_{\omega}^{n-1}(R)$, and hence $\mathrm{c} \operatorname{Tr}_{\omega} \cos \Omega^{n-1}(M) \in \omega_{S}{ }^{T_{n-1}}$. Thus from the exact sequences (4.2) and (4.3) we get the following exact sequence

$$
\operatorname{Tor}_{n+1}^{S}\left(\omega, c \operatorname{Tr}_{\omega} \operatorname{co} \Omega^{n}(M)\right) \rightarrow \operatorname{Tor}_{n-1}^{S}\left(\omega, \operatorname{Ext}_{R}^{n}(\omega, M)\right) \rightarrow 0 .
$$

By (1), we have E-cograde $\operatorname{Tor}_{n-1}^{S}\left(\omega, \operatorname{Ext}_{R}^{n}(\omega, M)\right) \geqslant n$. It follows from Lemma 4.11(2) that T-cograde $\omega \operatorname{Ext}_{R}^{n}(\omega, M) \geqslant n$. 
(2) $\Rightarrow(1)$ Let $N \in \operatorname{Mod} S$ and $X$ a quotient module of $\operatorname{Tor}_{n+1}^{S}(\omega, N)$ in $\operatorname{Mod} R$, and let $\beta: \operatorname{Tor}_{1}^{S}\left(\omega, \Omega_{\mathcal{F}}^{n}(N)\right)\left(\cong \operatorname{Tor}_{n+1}^{S}(\omega, N)\right) \rightarrow X$ be an epimorphism in $\operatorname{Mod} R$. By Lemma 3.1 (2), we have an exact sequence

$$
0 \rightarrow \operatorname{Im}\left(1_{\omega} \otimes f_{n+1}\right) \stackrel{\sigma}{\longrightarrow} \operatorname{acTr}_{\omega} \Omega_{\mathcal{F}}^{n}(N) \stackrel{\tau}{\longrightarrow} \operatorname{Tor}_{n+1}^{S}(\omega, N) \rightarrow 0
$$

in $\operatorname{Mod} R$ such that $\sigma_{*}$ is an isomorphism. Then we get an exact sequence

$$
0 \rightarrow \operatorname{Ker} f \stackrel{\eta}{\longrightarrow} \operatorname{ac} \operatorname{Tr}_{\omega} \Omega_{\mathcal{F}}^{n}(N) \stackrel{f}{\longrightarrow} X \rightarrow 0
$$

in $\operatorname{Mod} R$, where $f=\beta \cdot \tau$. It is easy to see that $\eta_{*}$ is an isomorphism.

Let $n=1$. Because $\Omega_{\mathcal{F}}^{1}(N) \in \operatorname{ac} \mathcal{T}_{\omega}^{1}(S)$ by [34, Corollary 3.5(1)], we have $\operatorname{acTr}_{\omega} \Omega_{\mathcal{F}}^{1}(N) \in{ }_{R} \omega^{\perp_{1}}$. Then the exact sequence (4.4) gives that $X_{*} \cong \operatorname{Ext}_{R}^{1}(\omega, \operatorname{Ker} f)$. So T-cograde $X_{*} \geqslant 1$ by (2), and hence E-cograde ${ }_{\omega} X \geqslant 1$ by Lemma 4.11(1). The case for $n=1$ is proved.

Now suppose $n \geqslant 2$. Then s.E-cograde $\operatorname{Tor}_{i+1}^{S}(\omega, N) \geqslant i$ for any $1 \leqslant i \leqslant$ $n-1$ and s.E-cograde ${ }_{\omega} \operatorname{Tor}_{n+1}^{S}(\omega, N) \geqslant n-1$ by the induction hypothesis. So E-cograde ${ }_{\omega} X \geqslant n-1$.

By Theorem 3.7, we have $\Omega_{\mathcal{F}}^{i}(S) \subseteq$ ac $\mathcal{T}_{\omega}^{i}(S)$ for any $1 \leqslant i \leqslant n$. So $\Omega_{\mathcal{F}}^{n}(N) \in$ $\operatorname{ac} \mathcal{T}_{\omega}^{n}(S)$ and $\operatorname{ac}_{\omega} \Omega_{\mathcal{F}}^{n}(N) \in{ }_{R} \omega^{\perp_{n}}$. It follows from the exact sequence (4.4) that $\operatorname{Ext}_{R}^{n-1}(\omega, X) \cong \operatorname{Ext}_{R}^{n}(\omega, \operatorname{Ker} f)$. Then by (2), we have T-cograde ${ }_{\omega} \operatorname{Ext}_{R}^{n-1}(\omega, X)=$

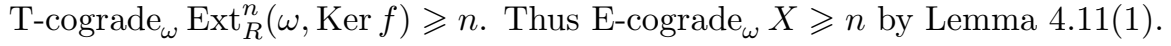

We also have the following

Proposition 4.13. For any $n \geqslant 1$, the following statements are equivalent.

(1) s.T-cograde ${ }_{\omega} \operatorname{Ext}_{S^{o p}}^{i+1}\left(\omega, N^{\prime}\right) \geqslant i$ for any $N^{\prime} \in \operatorname{Mod} S^{o p}$ and $1 \leqslant i \leqslant n$.

(2) E-cograde $_{\omega} \operatorname{Tor}_{i}^{R}\left(M^{\prime}, \omega\right) \geqslant i$ for any $M^{\prime} \in \operatorname{Mod} R^{o p}$ and $1 \leqslant i \leqslant n$.

(3) For any exact sequence

$$
0 \rightarrow A^{\prime} \rightarrow B^{\prime} \stackrel{f^{\prime}}{\longrightarrow} C^{\prime} \rightarrow 0
$$

in $\operatorname{Mod} S^{o p}$ with $A \in \Omega_{\mathcal{P}_{\omega}}^{i-1}\left(\cos _{\mathcal{P}_{\omega}}^{i}\left(S^{o p}\right)\right)$, $\operatorname{Tor}_{i}^{R}\left(\operatorname{Ext}_{S^{o p}}^{i}\left(\omega, f^{\prime}\right), \omega\right)$ is an epimorphism for any $0 \leqslant i \stackrel{\leqslant}{\leqslant}-1$.

(4) For any exact sequence

$$
0 \rightarrow A^{\prime} \rightarrow B^{\prime} \stackrel{f^{\prime}}{\longrightarrow} C^{\prime} \rightarrow 0
$$

in $\operatorname{Mod} S^{o p}$ with $A \in \Omega_{\mathcal{P}_{\omega}}^{i-1}\left(\cos \Omega^{i}\left(S^{o p}\right)\right)$, $\operatorname{Tor}_{i}^{R}\left(\operatorname{Ext}_{S^{o p}}^{i}\left(\omega, f^{\prime}\right), \omega\right)$ is an epimorphism for any $0 \leqslant i \leqslant n-1$.

(5) $\operatorname{Ext}_{S^{o p}}^{i}\left(\omega, \operatorname{Tor}_{i}^{R}\left(g^{\prime}, \omega\right)\right)$ is a monomorphism for any monomorphism $g^{\prime}$ : $B^{\prime} \longmapsto C^{\prime}$ in $\operatorname{Mod} R^{o p}$ with $B^{\prime}, C^{\prime} \in \Omega_{\mathcal{I}_{\omega}}^{1}\left(R^{o p}\right)$ and $0 \leqslant i \leqslant n-1$.

(6) $\operatorname{Ext}_{S^{o p}}^{i}\left(\omega, \operatorname{Tor}_{i}^{R}\left(g^{\prime}, \omega\right)\right)$ is a monomorphism for any monomorphism $g^{\prime}$ : $B^{\prime} \longmapsto C^{\prime}$ in $\operatorname{Mod} R^{o p}$ with $B^{\prime}, C^{\prime} \in \Omega_{\mathcal{F}}^{1}\left(R^{o p}\right)$ and $0 \leqslant i \leqslant n-1$.

(7) $\Omega_{\mathcal{F}}^{i}\left(R^{o p}\right) \subseteq \operatorname{ac} \mathcal{T}_{\omega}^{i+1}\left(R^{o p}\right)$ for any $1 \leqslant i \leqslant n$.

Proof. By the symmetric versions of Theorems 3.8 and 3.7, we have (1) $\Leftrightarrow(3) \Leftrightarrow$ (4) and $(2) \Leftrightarrow(5) \Leftrightarrow(6) \Leftrightarrow(7)$ respectively. In the following, we will prove (1) $\Leftrightarrow(2)$ by induction on $n$.

$(1) \Rightarrow(2)$ Let $M^{\prime} \in \operatorname{Mod} R^{o p}$ and let

$$
\cdots \rightarrow F_{i+1}\left(M^{\prime}\right) \stackrel{f_{i}}{\longrightarrow} F_{i}\left(M^{\prime}\right) \rightarrow \cdots \stackrel{f_{0}}{\longrightarrow} F_{0}\left(M^{\prime}\right) \rightarrow M^{\prime} \rightarrow 0
$$


be the minimal flat resolution of $M^{\prime}$ in $\operatorname{Mod} R^{o p}$. By Lemma 3.1(2), for any $n \geqslant 1$, there exist exact sequences

$$
\begin{gathered}
0 \rightarrow \operatorname{Im}\left(1_{\omega} \otimes f_{n}\right) \stackrel{\sigma}{\longrightarrow} \operatorname{acTr}_{\omega} \Omega_{\mathcal{F}}^{n-1}\left(M^{\prime}\right) \stackrel{\tau}{\longrightarrow} \operatorname{Tor}_{n}^{R}\left(M^{\prime}, \omega\right) \rightarrow 0, \\
0 \rightarrow \operatorname{acTr}_{\omega} \Omega_{\mathcal{F}}^{n}\left(M^{\prime}\right) \rightarrow F_{n+1}\left(M^{\prime}\right) \otimes_{R} \omega \rightarrow \operatorname{Im}\left(1_{\omega} \otimes f_{n}\right) \rightarrow 0
\end{gathered}
$$

in Mod $S^{o p}$ such that $\sigma_{*}$ is an isomorphism. Because $F_{n+1}\left(M^{\prime}\right) \otimes_{R} \omega \in \omega_{S}{ }^{\perp}$ by [19, Corollary 6.1], it follows from the exact sequence (4.6) that $\operatorname{Ext}_{S^{o p}}^{i}\left(\omega, \operatorname{Im}\left(1_{\omega} \otimes f_{n}\right)\right) \cong$ $\operatorname{Ext}_{S^{o p}}^{i+1}\left(\omega, \operatorname{acTr}_{\omega} \Omega_{\mathcal{F}}^{n}\left(M^{\prime}\right)\right)$ for any $i \geqslant 1$.

If $n=1$, then from the exact sequence (4.5) we get an exact sequence

$$
0 \rightarrow\left(\operatorname{Tor}_{1}^{R}\left(M^{\prime}, \omega\right)\right)_{*} \rightarrow \operatorname{Ext}_{S^{o p}}^{2}\left(\omega, \operatorname{acTr}_{\omega} \Omega_{\mathcal{F}}^{1}\left(M^{\prime}\right)\right)\left(\cong \operatorname{Ext}_{S^{o p}}^{1}\left(\omega, \operatorname{Im}\left(1_{\omega} \otimes f_{n}\right)\right)\right)
$$

in $\operatorname{Mod} R^{o p}$. Because s.T-cograde $\operatorname{Ext}_{S^{o p}}^{2}\left(\omega, \operatorname{acTr}_{\omega} \Omega_{\mathcal{F}}^{1}\left(M^{\prime}\right)\right) \geqslant 1$ by assumption, we have T-cograde ${ }_{\omega}\left(\operatorname{Tor}_{1}^{R}\left(M^{\prime}, \omega\right)\right)_{*} \geqslant 1$. It is derived from Lemma 4.11(1) that E-cograde $\operatorname{Tor}_{1}^{R}\left(M^{\prime}, \omega\right) \geqslant 1$.

Now suppose $n \geqslant 2$. Then E-cograde $\operatorname{Tor}_{i}^{R}\left(M^{\prime}, \omega\right) \geqslant i$ for any $1 \leqslant i \leqslant n-1$ and E-cograde ${ }_{\omega} \operatorname{Tor}_{n}^{R}\left(M^{\prime}, \omega\right) \geqslant n-1$ by the induction hypothesis. It follows from Theorem 3.7 that $\Omega_{\mathcal{F}}^{i}\left(R^{o p}\right) \subseteq$ ac $\mathcal{T}_{\omega}^{i}\left(R^{o p}\right)$ for any $1 \leqslant i \leqslant n$. So $\Omega_{\mathcal{F}}^{n-1}\left(M^{\prime}\right) \in$ $\operatorname{ac} \mathcal{T}_{\omega}^{n-1}\left(R^{o p}\right)$ and $\operatorname{ac} \operatorname{Tr}_{\omega} \Omega_{\mathcal{F}}^{n-1}\left(M^{\prime}\right) \in \omega_{S}{ }^{\perp_{n-1}}$. Thus from the exact sequences (4.5) and (4.6) we get the following exact sequence

$$
0 \rightarrow \operatorname{Ext}_{S^{o p}}^{n-1}\left(\omega, \operatorname{Tor}_{n}^{R}\left(M^{\prime}, \omega\right)\right) \rightarrow \operatorname{Ext}_{S^{o p}}^{n+1}\left(\omega, \operatorname{acTr}_{\omega} \Omega_{\mathcal{F}}^{n}\left(M^{\prime}\right)\right) .
$$

By (1), we have T-cograde ${ }_{\omega} \operatorname{Ext}_{S^{o p}}^{n-1}\left(\omega, \operatorname{Tor}_{n}^{R}\left(M^{\prime}, \omega\right)\right) \geqslant n$. It follows from Lemma4.11(1) that E-cograde $\operatorname{Tor}_{n}^{R}\left(M^{\prime}, \omega\right) \geqslant n$.

$(2) \Rightarrow(1)$ Let $N^{\prime} \in \operatorname{Mod} S^{o p}$ and $Y$ a submodule of $\operatorname{Ext}_{S^{o p}}^{n+1}\left(\omega, N^{\prime}\right)$ in $\operatorname{Mod} R^{o p}$, and let $\alpha: Y \longmapsto \operatorname{Ext}_{S^{o p}}^{1}\left(\omega, \operatorname{co}^{n}\left(N^{\prime}\right)\right)\left(\cong \operatorname{Ext}_{S^{\circ p}}^{n+1}\left(\omega, N^{\prime}\right)\right)$ be a monomorphism in Mod $R^{o p}$. By Lemma 3.1(1), we have an exact sequence

$$
0 \rightarrow \operatorname{Ext}_{S^{o p}}^{n+1}\left(\omega, N^{\prime}\right) \stackrel{\lambda}{\longrightarrow} \operatorname{cTr}_{\omega} \cos ^{n}\left(N^{\prime}\right) \stackrel{\pi}{\longrightarrow} I^{n+1}\left(N^{\prime}\right)_{*} / \operatorname{co} \Omega^{n+1}\left(N^{\prime}\right)_{*} \rightarrow 0
$$

in $\operatorname{Mod} R^{o p}$ such that $\pi \otimes 1_{\omega}$ is an isomorphism. Then we get an exact sequence

$$
0 \rightarrow Y \stackrel{g}{\longrightarrow} \mathrm{cTr}_{\omega} \operatorname{co} \Omega^{n}\left(N^{\prime}\right) \stackrel{\rho}{\longrightarrow} \text { Coker } g \rightarrow 0
$$

in $\operatorname{Mod} R^{o p}$, where $g=\lambda \cdot \alpha$. It is easy to see that $\rho \otimes 1_{\omega}$ is an isomorphism.

Let $n=1$. Because $\cos \Omega^{1}\left(N^{\prime}\right) \in \mathrm{c} \mathcal{T}_{\omega}^{1}\left(S^{o p}\right)$ by [32, Lemma 2.5(2)], we have $\operatorname{cTr}_{\omega} \operatorname{co} \Omega^{1}\left(N^{\prime}\right) \in \omega_{S}{ }^{T_{1}}$. Then the exact sequence (4.7) gives that $Y \otimes_{R} \omega \cong$ $\operatorname{Tor}_{1}^{R}($ Coker $g, \omega)$. So E-cograde ${ }_{\omega} Y \otimes_{R} \omega \geqslant 1$ by (2), and hence T-cograde ${ }_{\omega} Y \geqslant 1$ by Lemma 4.11(2). The case for $n=1$ is proved.

Now suppose $n \geqslant 2$. Then s.T-cograde $\operatorname{Ext}_{S^{o p}}^{i+1}\left(\omega, N^{\prime}\right) \geqslant i$ for any $1 \leqslant i \leqslant$ $n-1$ and s.T-cograde $\operatorname{Ext}_{S^{o p}}^{n+1}\left(\omega, N^{\prime}\right) \geqslant n-1$ by the induction hypothesis. So T-cograde ${ }_{\omega} Y \geqslant n-1$.

By Theorem 3.5 we have $\operatorname{co} \Omega^{i}\left(R^{o p}\right) \subseteq \mathrm{c} \mathcal{T}_{\omega}^{i}\left(R^{o p}\right)$ for any $1 \leqslant i \leqslant n$. So $\cos ^{n}\left(N^{\prime}\right) \in \mathrm{c} \mathcal{T}_{\omega}^{i}\left(R^{o p}\right)$ and $\operatorname{cTr}_{\omega} \cos \Omega^{n}\left(N^{\prime}\right) \in{ }_{R} \omega^{\top_{n}}$. It follows from the exact sequence (4.7) that $\operatorname{Tor}_{n-1}^{R}(Y, \omega) \cong \operatorname{Tor}_{n}^{R}(\operatorname{Coker} g, \omega)$. Then by $(2)$, we have E-cograde $\operatorname{Cor}_{n-1}^{R}(Y, \omega)=$ E-cograde $_{\omega} \operatorname{Tor}_{n}^{R}($ Coker $g, \omega) \geqslant n$. Thus T-cograde $\omega \geqslant n$ by Lemma 4.11(2).

Now we are in a position to state the following

Theorem 4.14. Let $R$ be semiregular and $n \geqslant 1$. Then the following statements are equivalent.

(1) $\operatorname{pd}_{S^{o p}} P_{i}(\omega)^{*} \leqslant i+1$ for any $0 \leqslant i \leqslant n-1$. 
(2) $\mathcal{P}_{\omega}(R)-\operatorname{id}_{R} P_{i}(\omega) \leqslant i+1$ for any $0 \leqslant i \leqslant n-1$.

(3) s.T-cograde ${ }_{\omega} \operatorname{Ext}_{S o p}^{i+1}\left(\omega, N^{\prime}\right) \geqslant i$ for any $N^{\prime} \in \operatorname{Mod} S^{o p}$ and $1 \leqslant i \leqslant n$.

(4) s.E-cograde $\operatorname{Tor}_{i+1}^{S}(\omega, N) \geqslant i$ for any $N \in \operatorname{Mod} S$ and $1 \leqslant i \leqslant n$.

(5) T-cograde $\operatorname{Ext}_{R}^{i}(\omega, M) \geqslant i$ for any $M \in \operatorname{Mod} R$ and $1 \leqslant i \leqslant n$.

(6) E-cograde $\operatorname{Tor}_{i}^{R}\left(M^{\prime}, \omega\right) \geqslant i$ for any $M^{\prime} \in \operatorname{Mod} R^{o p}$ and $1 \leqslant i \leqslant n$.

(7) $\operatorname{Tor}_{i}^{S}\left(\omega, \operatorname{Ext}_{R}^{i}(\omega, f)\right)$ is an epimorphism for any epimorphism $f: B \rightarrow C$ in $\operatorname{Mod} R$ with $B, C \in \operatorname{co} \Omega^{1}(R)$ and $0 \leqslant i \leqslant n-1$.

(8) $\operatorname{Ext}_{S^{\circ o p}}^{i}\left(\omega, \operatorname{Tor}_{i}^{R}\left(f^{\prime}, \omega\right)\right)$ is a monomorphism for any monomorphism $f^{\prime}$ : $B^{\prime} \longmapsto C^{\prime}$ in $\operatorname{Mod} R^{o p}$ with $B^{\prime}, C^{\prime} \in \Omega_{\mathcal{F}}^{1}\left(R^{o p}\right)$ and $0 \leqslant i \leqslant n-1$.

(9) For any exact sequence

$$
0 \rightarrow A^{\prime} \rightarrow B^{\prime} \stackrel{g^{\prime}}{\rightarrow} C^{\prime} \rightarrow 0
$$

in $\operatorname{Mod} S^{o p}$ with $A^{\prime} \in \Omega_{\mathcal{P}_{\omega}}^{i-1}\left(\operatorname{co} \Omega^{i}\left(S^{o p}\right)\right)$, $\operatorname{Tor}_{i}^{R}\left(\operatorname{Ext}_{S^{o p}}^{i}\left(\omega, g^{\prime}\right), \omega\right)$ is an epimorphism for any $0 \leqslant i \leqslant n-1$.

(10) For any exact sequence

$$
0 \rightarrow A \stackrel{g}{\longrightarrow} B \rightarrow C \rightarrow 0
$$

in $\operatorname{Mod} S$ with $C \in \operatorname{co} \Omega_{\mathcal{I}_{\omega}}^{i-1}\left(\Omega_{\mathcal{F}}^{i}(S)\right)$, $\operatorname{Ext}_{R}^{i}\left(\omega, \operatorname{Tor}_{i}^{S}(\omega, g)\right)$ is a monomorphism for any $0 \leqslant i \leqslant n-1$.

(11) $\cos \Omega^{i}(R) \subseteq \mathrm{c} \mathcal{T}_{\omega}^{i+1}(R)$ for any $1 \leqslant i \leqslant n$

(12) $\Omega_{\mathcal{F}}^{i}\left(R^{o p}\right) \subseteq \operatorname{ac}_{\omega}^{i+1}\left(R^{o p}\right)$ for any $1 \leqslant i \leqslant n$.

Proof. By Proposition 4.7, we have $(1) \Leftrightarrow(2) \Leftrightarrow(3) \Leftrightarrow(4)$. By Propositions 4.12 and 4.13 , we have $(4) \Leftrightarrow(5) \Leftrightarrow(7) \Leftrightarrow(10) \Leftrightarrow(11)$ and $(3) \Leftrightarrow(6) \Leftrightarrow(8) \Leftrightarrow(9) \Leftrightarrow$ (12) respectively.

For the right quasi 1-cograde condition, we have some additional interesting equivalent characterizations.

Proposition 4.15. Let $R$ be a semiregular ring. Then the following statements are equivalent.

(1) $\operatorname{pd}_{S^{o p}} P_{0}(\omega)^{*} \leqslant 1$.

(2) s.E-cograde $\operatorname{Tor}_{2}^{S}(\omega, N) \geqslant 1$ for any $N \in \operatorname{Mod} S$.

(3) $\theta_{M}$ is a superfluous epimorphism for any $M \in \operatorname{co} \Omega^{1}(R)$.

(4) $\mu_{M^{\prime}}$ is an essential monomorphism for any $M^{\prime} \in \Omega_{\mathcal{F}}^{1}\left(R^{o p}\right)$.

Proof. By Theorem 4.14, we have $(1) \Leftrightarrow(2)$.

$(1) \Rightarrow(3)$ Let $M \in \operatorname{co} \Omega^{1}(R)$. By [32, Lemma 2.5(2)], we have $\cos \Omega^{1}(R) \subseteq$ $c \mathcal{T}_{\omega}^{1}(R)$. So $M \in \mathrm{c} \mathcal{T}_{\omega}^{1}(R)$ and $\theta_{M}$ is an epimorphism. Because $\operatorname{Ker} \theta_{M} \cong$ $\operatorname{Tor}_{2}^{S}\left(\omega, c \operatorname{Tr}_{\omega} M\right)$ by [32, Proposition 3.2], we have

$$
\operatorname{Hom}_{R}\left(P_{0}(\omega), \operatorname{Ker} \theta_{M}\right) \cong \operatorname{Hom}_{R}\left(P_{0}(\omega), \operatorname{Tor}_{2}^{S}\left(\omega, c \operatorname{Tr}_{\omega} M\right)\right)=0
$$

by (1) and Lemma 4.6. It follows easily that $X_{*}=0$ for any quotient module $X$ of $\operatorname{Ker} \theta_{M}$. Let $A$ be a submodule of $\omega \otimes_{S} M_{*}$ in $\operatorname{Mod} R$ such that $\operatorname{Ker} \theta_{M}+$ $A=\omega \otimes_{S} M_{*}$. Then $\left(\operatorname{Ker} \theta_{M}+A\right) / A\left(\cong \operatorname{Ker} \theta_{M} /\left(A \cap \operatorname{Ker} \theta_{M}\right)\right)$ is isomorphic to a quotient module of $\operatorname{Ker} \theta_{M}$, and so $\left(\left(\operatorname{Ker} \theta_{M}+A\right) / A\right)_{*}=0$. Since $\omega \otimes_{S} M_{*} \in$ $\mathrm{c} \mathcal{T}_{\omega}^{1}(R)$ by [33, Lemma $\left.6.1(2)\right],\left(\operatorname{Ker} \theta_{M}+A\right) / A \in \mathrm{c} \mathcal{T}_{\omega}^{1}(R)$ by [32, Corollary 3.8]. It follows that $\theta_{\left(\operatorname{Ker} \theta_{M}+A\right) / A}: \omega \otimes_{S}\left(\left(\operatorname{Ker} \theta_{M}+A\right) / A\right)_{*} \rightarrow\left(\operatorname{Ker} \theta_{M}+A\right) / A$ is epic and $\left(\operatorname{Ker} \theta_{M}+A\right) / A=0$. It induces that $A=\operatorname{Ker} \theta_{M}+A=\omega \otimes_{S} M_{*}$ and $\theta_{M}$ is a superfluous epimorphism. 
$(3) \Rightarrow(2)$ Let $f: B \rightarrow C$ be an epimorphism in $\operatorname{Mod} R$ with $B, C \in \operatorname{co} \Omega^{1}(R)(\subseteq$ $\left.c \mathcal{T}_{\omega}^{1}(R)\right)$. Then $\theta_{C} \cdot\left(1_{\omega} \otimes f_{*}\right)=f \cdot \theta_{B}$ is epic. Because $\theta_{C}$ is a superfluous epimorphism by (3), it follows from [1, Corollary 5.15] that $1_{\omega} \otimes f_{*}$ is epic. Now the assertion follows from Theorem 4.14 .

$(1) \Rightarrow(4)$ Let $M^{\prime} \in \Omega_{\mathcal{F}}^{1}\left(R^{o p}\right)$. By [34, Corollary 3.5(1)], we have $\Omega_{\mathcal{F}}^{1}\left(R^{o p}\right) \subseteq$ $\operatorname{ac} \mathcal{T}_{\omega}^{1}\left(R^{o p}\right)$. So $M^{\prime} \in \operatorname{ac} \mathcal{T}_{\omega}^{1}\left(R^{o p}\right)$ and $\mu_{M^{\prime}}$ is a monomorphism. Because Coker $\mu_{M^{\prime}} \cong$ $\operatorname{Ext}_{S^{o p}}^{2}\left(\omega, \operatorname{acTr}_{\omega} M^{\prime}\right)$ by [34, Proposition 3.2], we have

$$
\text { Coker } \mu_{M^{\prime}} \otimes_{R} P_{0}(\omega) \cong \operatorname{Ext}_{S^{o p}}^{2}\left(\omega, \operatorname{acTr}_{\omega} M^{\prime}\right) \otimes_{R} P_{0}(\omega)=0
$$

by (1) and Lemma 4.6. It follows easily that $Y \otimes_{R} \omega=0$ for any submodule $Y$ of Coker $\mu_{M^{\prime}}$. Let $A^{\prime}$ be a submodule of $\left(M^{\prime} \otimes_{R} \omega\right)_{*}$ in $\operatorname{Mod} R^{o p}$ with $A^{\prime} \cap M^{\prime}=0$. Then $A^{\prime} \cong A^{\prime} / A^{\prime} \cap M^{\prime} \cong\left(A^{\prime}+M^{\prime}\right) / M^{\prime}$ is isomorphic to a submodule of Coker $\mu_{M^{\prime}}$, and so $A^{\prime} \otimes_{R} \omega=0$. Since $\left(M^{\prime} \otimes_{R} \omega\right)_{*} \in \operatorname{ac} \mathcal{T}_{\omega}^{1}\left(R^{o p}\right)$ by [33, Lemma 6.1(1)], $A^{\prime} \in \operatorname{ac} \mathcal{T}_{\omega}^{1}\left(R^{o p}\right)$ by [34, Corollary 3.3(1)]. It follows that $\mu_{A^{\prime}}: A^{\prime} \rightarrow\left(A^{\prime} \otimes_{R} \omega\right)_{*}$ is monic, It induces that $A^{\prime}=0$ and $\mu_{M^{\prime}}$ is an essential monomorphism.

$(4) \Rightarrow(2)$ Let $g: B^{\prime} \rightarrow C^{\prime}$ be a monomorphism in Mod $R^{o p}$ with $B^{\prime}, C^{\prime} \in$ $\Omega_{\mathcal{F}}^{1}\left(R^{o p}\right)\left(\subseteq \operatorname{ac} \mathcal{T}_{\omega}^{1}\left(R^{o p}\right)\right)$. Then $\left(g \otimes 1_{\omega}\right)_{*} \cdot \mu_{B^{\prime}}=\mu_{C^{\prime}} \cdot g$ is monic. Because $\mu_{B^{\prime}}$ is an essential monomorphism by (4), it follows from [1, Corollary 5.13] that $\left(g \otimes 1_{\omega}\right)_{*}$ is monic. Now the assertion follows from Theorem 4.14.

\subsection{The equivalence of certain cograde condition of modules}

We have the following facts: for the strong Tor-cograde condition of modules in Theorem 3.8(1) and the strong Ext-cograde condition of modules in Theorem 3.9(1), they are equivalent when $k=0$ by Theorems 4.8. but they are not equivalent when $k=1$ by Theorem 4.14 and Subsection 4.4 below. Also from Theorem 4.14 and Subsection 4.4 below we know that the Tor-cograde condition of modules in Theorem 3.5(1) and the Ext-cograde condition of modules in Theorem 3.7(1) are not equivalent when $k=0$. In this subsection, we will show that these two cograde conditions of modules are equivalent when $k=1$.

For any $i \geqslant 1$, by [34, Proposition 3.8] we have ac $\mathcal{T}_{\omega}^{i}(S) \subseteq \Omega_{\mathcal{I}_{\omega}}^{i}(S)$. The following result characterizes when they are identical.

Proposition 4.16. For any $n \geqslant 1$, the following statements are equivalent.

(1) E-cograde $\operatorname{Tor}_{i}^{S}(\omega, N) \geqslant i-1$ for any $N \in \operatorname{co} \Omega_{A}^{i}(S)$ and $1 \leqslant i \leqslant n$.

(2) E-cograde $_{\omega} \operatorname{Tor}_{i}^{S}(\omega, N) \geqslant i-1$ for any $N \in \cos _{\mathcal{I}_{\omega}}^{i}(S)$ and $1 \leqslant i \leqslant n$.

(3) $\operatorname{ac}^{i} \mathcal{T}_{\omega}^{i}(S)=\Omega_{\mathcal{A}}^{i}(S)$ for any $1 \leqslant i \leqslant n$.

(4) $\operatorname{ac} \mathcal{T}_{\omega}^{i}(S)=\Omega_{\mathcal{I}_{\omega}}^{i}(S)$ for any $1 \leqslant i \leqslant n$.

Proof. Because $\mathcal{I}_{\omega}(S) \subseteq \mathcal{A}_{\omega}(S)$, we have (1) $\Rightarrow(2)$. By Lemma 3.6(2), we have (3) $\Leftrightarrow(4)$.

$(2) \Rightarrow(4)$ By [34, Proposition 3.8], it suffices to prove $\Omega_{\mathcal{I}_{\omega}}^{i}(S) \subseteq$ ac $\mathcal{T}_{\omega}^{i}(S)$ for any $1 \leqslant i \leqslant n$. We proceed by induction on $n$. The case for $n=1$ follows from Lemma $2.9(1)$.

Now let $N \in \Omega_{\mathcal{I}_{\omega}}^{n}(S)$ with $n \geqslant 2$ and let

$$
0 \longrightarrow N \stackrel{f^{0}}{\longrightarrow} I^{0} \stackrel{f^{1}}{\longrightarrow} \cdots \stackrel{f^{n-1}}{\longrightarrow} I^{n-1}
$$

be an exact sequence in $\operatorname{Mod} S$ with all $I^{i}$ in $\mathcal{I}_{\omega}(S)$. By the induction hypothesis, we have $\operatorname{Im} f^{1} \in \operatorname{ac} \mathcal{T}_{\omega}^{n-1}(S)$. Applying the functor $\omega \otimes_{S}-$ to (4.8) gives an exact 
sequence

$$
0 \rightarrow \operatorname{Tor}_{n}^{S}\left(\omega, \text { Coker } f^{n-1}\right) \longrightarrow \omega \otimes_{S} N \stackrel{1_{\omega} \otimes f^{0}}{\longrightarrow} \omega \otimes_{S} I^{0} \longrightarrow \omega \otimes_{S} \operatorname{Im} f^{1} \rightarrow 0
$$

in $\operatorname{Mod} R$. Set $M:=\operatorname{Im}\left(1_{\omega} \otimes f^{0}\right)$ and let $1_{\omega} \otimes f^{0}:=\alpha \cdot \pi\left(\right.$ where $\pi: \omega \otimes \otimes_{S} N \rightarrow M$ and $\alpha: M \hookrightarrow \omega \otimes_{S} I^{0}$ ) be the natural epic-monic decomposition of $1_{\omega} \otimes f^{0}$. Then we have the following commutative diagram with exact rows

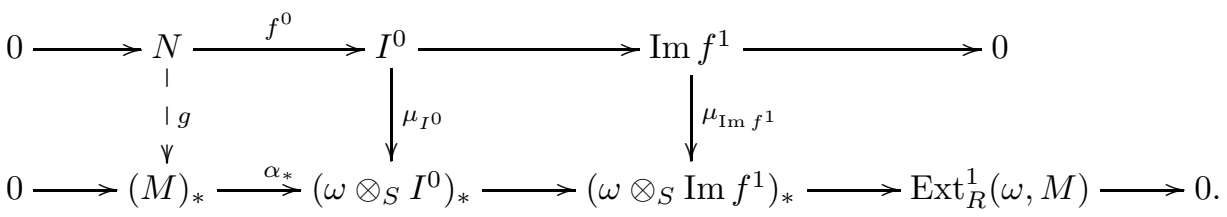

Diagram (4.10)

Since $\mu_{\operatorname{Im} f^{1}}$ is a monomorphism by the above argument, it follows from the snake lemma that $g$ is an epimorphism. On the other hand, we have

$$
\alpha_{*} \cdot \pi_{*} \cdot \mu_{N}=(\alpha \cdot \pi)_{*} \cdot \mu_{N}=\left(1_{\omega} \otimes f^{0}\right)_{*} \cdot \mu_{N}=\mu_{I^{0}} \cdot f^{0}=\alpha_{*} \cdot g .
$$

As $\alpha_{*}$ is monic, we get that $\pi_{*} \cdot \mu_{N}=g$ and $\pi_{*}$ is epic. Consider the following commutative diagram with exact rows

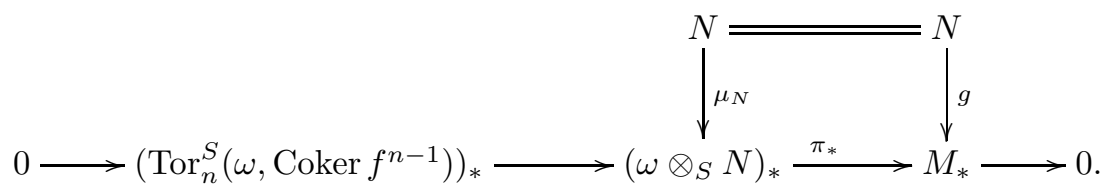

Diagram (4.11)

Because $\left(\operatorname{Tor}_{n}^{S}\left(\omega, \text { Coker } f^{n-1}\right)\right)_{*}=0$ by assumption, we have that $\pi_{*}$ is an isomorphism. So $\mu_{N}$ is epic by the diagram (4.11), and hence an isomorphism. Thus $N \in \operatorname{ac}_{\omega}^{2}(S)$ and the case for $n=2$ follows.

Now suppose $n \geqslant 3$. By the induction hypothesis, we have that $\operatorname{Im} f^{1} \in$ $\operatorname{ac} \mathcal{T}_{\omega}^{n-1}(S)$ and $\mu_{\operatorname{Im} f^{1}}$ is an isomorphism. So $\operatorname{Ext}_{R}^{1}(\omega, M)=0$ by the diagram (4.10). In addition, we have $\omega \otimes_{S} \operatorname{Im} f^{1} \in R^{\omega^{\perp_{n-3}}}$ by [34, Corollary 3.3(3)]. Because E-cograde ${ }_{\omega} \operatorname{Tor}_{n}^{S}\left(\omega\right.$, Coker $\left.f^{n-1}\right) \geqslant n-1$ (by assumption) and $\omega \otimes_{S} I^{0} \in{ }_{R} \omega^{\perp}$, applying the dimension shifting to (4.9) we obtain $\omega \otimes_{S} N \in R_{R} \omega^{\perp_{n-2}}$. Therefore we conclude that $N \in \operatorname{ac} \mathcal{T}_{\omega}^{n}(S)$ by [34, Corollary 3.3(3)] again.

$(3) \Rightarrow(1)$ We proceed by induction on $n$. The case for $n=1$ is trivial. Let $N \in \operatorname{co} \Omega_{\mathcal{A}}^{n}(S)$ with $n \geqslant 2$. Then there exists an exact sequence

$$
0 \rightarrow H \rightarrow A_{n-1} \stackrel{f}{\longrightarrow} A_{n-2} \rightarrow \cdots \rightarrow A_{0} \rightarrow N \rightarrow 0
$$

in $\operatorname{Mod} S$ with all $A_{i}$ in $\mathcal{A}_{\omega}(S)$. By (3), we have $H \in \operatorname{ac} \mathcal{T}_{\omega}^{n}(S)$. By the induction hypothesis, we have that E-cograde $\operatorname{Tor}_{i}^{S}(\omega, N) \geqslant i-1$ for any $1 \leqslant i \leqslant n-1$ and E-cograde $_{\omega} \operatorname{Tor}_{n}^{S}(\omega, N) \geqslant n-2$.

Put $M:=\operatorname{Ker}\left(1_{\omega} \otimes f\right)$. Because $A_{i} \in \operatorname{ac} \mathcal{T}_{\omega}(S)$ by [34, Theorem 3.11(1)], we obtain that $M_{*} \cong H\left(\in \operatorname{ac} \mathcal{T}_{\omega}^{n}(S)\right)$ and $M \in R_{\omega^{\perp_{n-2}}}$. By [35. Proposition 5.1], we 
have the following exact sequences

$$
\begin{gathered}
0 \rightarrow \operatorname{Tor}_{n}^{S}(\omega, N)\left(\cong \operatorname{Tor}_{2}^{S}(\omega, \text { Coker } f)\right) \rightarrow \omega \otimes_{S} M_{*} \stackrel{\pi}{\longrightarrow} \operatorname{Im} \theta_{M} \rightarrow 0, \\
0 \rightarrow \operatorname{Im} \theta_{M} \stackrel{\lambda}{\longrightarrow} M \rightarrow \operatorname{Tor}_{n-1}^{S}(\omega, N)\left(\cong \operatorname{Tor}_{1}^{S}(\omega, \text { Coker } f)\right) \rightarrow 0
\end{gathered}
$$

such that $\theta_{M}=\lambda \cdot \pi$. Since $\mu_{M_{*}}$ is an isomorphism, it follows from 33, Lemma 6.1(1)] that $\left(\theta_{M}\right)_{*}$ is also an isomorphism. Then both $\lambda_{*}$ and $\pi_{*}$ are isomorphisms.

From the exact sequence (4.13), we get $\operatorname{Im} \theta_{M} \in{ }_{R} \omega^{\perp_{n-2}}$. Because $\omega \otimes_{S} M_{*} \in$ ${ }_{R} \omega^{\perp_{n-2}}$ by [34. Corollary 3.3], from the exact sequence (4.12) it yields that $\operatorname{Ext}_{R}^{n-2}\left(\omega, \operatorname{Tor}_{n}^{S}(\omega, N)\right)=0$. Thus we have E-cograde $\omega \operatorname{Tor}_{n}^{S}(\omega, N) \geqslant n-1$.

For any $i \geqslant 1$, by [32, Proposition 3.7] we have $\mathrm{c} \mathcal{T}_{\omega}^{i}(R) \subseteq \operatorname{co} \Omega_{\mathcal{P}_{\omega}}^{i}(R)$. The following result characterizes when they are identical.

Proposition 4.17. For any $n \geqslant 1$, the following statements are equivalent.

(1) T-cograde $\operatorname{Ext}_{R}^{i}(\omega, M) \geqslant i-1$ for any $M \in \Omega_{\mathcal{B}}^{i}(R)$ and $1 \leqslant i \leqslant n$.

(2) $\mathrm{T}$-cograde ${ }_{\omega} \operatorname{Ext}_{R}^{i}(\omega, M) \geqslant i-1$ for any $M \in \Omega_{\mathcal{F}_{\omega}}^{i}(R)$ and $1 \leqslant i \leqslant n$.

(3) T-cograde ${ }_{\omega} \operatorname{Ext}_{R}^{i}(\omega, M) \geqslant i-1$ for any $M \in \Omega_{\mathcal{P}_{\omega}}^{i}(R)$ and $1 \leqslant i \leqslant n$.

(4) $c \mathcal{T}_{\omega}^{i}(R)=\operatorname{co} \Omega_{\mathcal{B}}^{i}(R)$ for any $1 \leqslant i \leqslant n$.

(5) $\mathrm{c} \mathcal{T}_{\omega}^{i}(R)=\operatorname{co} \Omega_{\mathcal{F}_{\omega}}^{i}(R)$ for any $1 \leqslant i \leqslant n$.

(6) $\mathrm{c} \mathcal{T}_{\omega}^{i}(R)=\operatorname{co} \Omega_{\mathcal{P}_{\omega}}^{i}(R)$ for any $1 \leqslant i \leqslant n$.

Proof. Because $\mathcal{B}_{\omega}(R) \supseteq \mathcal{F}_{\omega}(R) \supseteq \mathcal{P}_{\omega}(R)$, we have (1) $\Rightarrow(2) \Rightarrow$ (3). By Lemma 3.4(2), we have $(4) \Leftrightarrow(5) \Leftrightarrow(6)$.

$(3) \Rightarrow(6)$ By [32, Proposition 3.7], it suffices to prove $\operatorname{co} \Omega_{\mathcal{P}_{\omega}}^{i}(R) \subseteq \mathrm{c} \mathcal{T}_{\omega}^{i}(R)$ for any $1 \leqslant i \leqslant n$. We proceed by induction on $n$. The case for $n=1$ follows from Lemma 2.9 $(2)$.

Now let $M \in \cos \Omega_{\mathcal{P}_{\omega}}^{n}(R)$ with $n \geqslant 2$ and let

$$
W_{n-1} \stackrel{f_{n-1}}{\longrightarrow} \cdots \rightarrow W_{1} \stackrel{f_{1}}{\longrightarrow} W_{0} \stackrel{f_{0}}{\longrightarrow} M \rightarrow 0
$$

be an exact sequence in $\operatorname{Mod} R$ with all $W_{i}$ in $\mathcal{P}_{\omega}(R)$. By the induction hypothesis, we have $\operatorname{Im} f_{1} \in \mathrm{c} \mathcal{T}_{\omega}^{n-1}(R)$. Applying the functor $(-)_{*}$ to (4.14) gives an exact sequence

$$
0 \rightarrow\left(\operatorname{Im} f_{1}\right)_{*} \rightarrow W_{0 *} \stackrel{f_{0_{*}}}{\rightarrow} M_{*} \rightarrow \operatorname{Ext}_{R}^{n}\left(\omega, \operatorname{Ker} f_{n-1}\right) \rightarrow 0 .
$$

Set $N:=\operatorname{Im}\left(f_{0_{*}}\right)$ and let $f_{0_{*}}:=\alpha \cdot \pi$ (where $\pi: W_{0_{*}} \rightarrow N$ and $\alpha: N \hookrightarrow M_{*}$ ) be the natural epic-monic decompositions of $f_{0 *}$. Then we have the following commutative diagram with exact rows

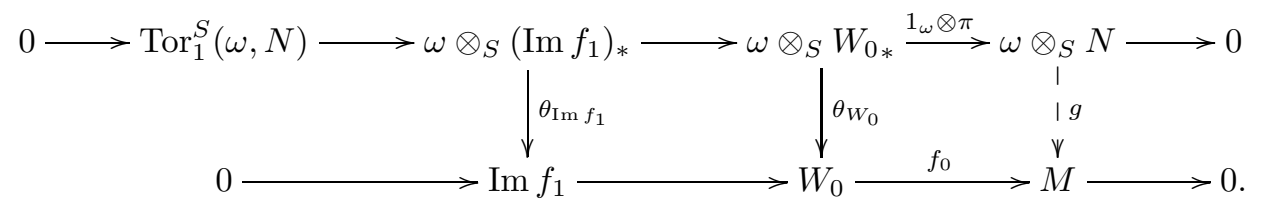

Diagram (4.16)

So we have

$$
\theta_{M} \cdot\left(1_{\omega} \otimes \alpha\right) \cdot\left(1_{\omega} \otimes \pi\right)=\theta_{M} \cdot\left(1_{\omega} \otimes f_{0 *}\right)=f_{0} \cdot \theta_{W_{0}}=g \cdot\left(1_{\omega} \otimes \pi\right) .
$$


Because $1_{\omega} \otimes \pi$ is epic, we have $\theta_{M} \cdot\left(1_{\omega} \otimes \alpha\right)=g$ and the following commutative diagram with exact rows

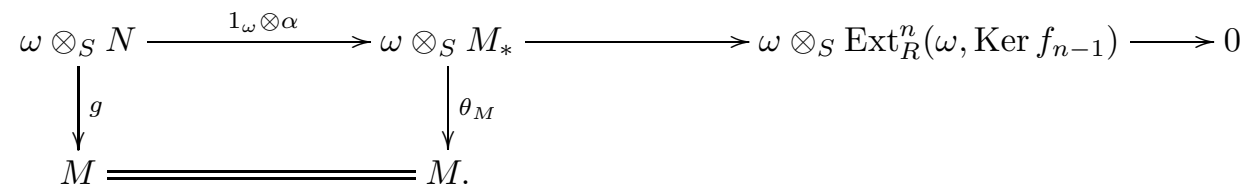

\section{Diagram (4.17)}

Since $\theta_{\operatorname{Im} f_{1}}$ is an epimorphism by the above argument, it follows from the snake lemma that $g$ is an isomorphism. Thus $1_{\omega} \otimes \alpha$ is a monomorphism. Because $\omega \otimes_{S} \operatorname{Ext}_{R}^{n}\left(\omega, \operatorname{Ker} f_{n-1}\right)=0$ by assumption, we have that $\theta_{M}$ is an isomorphism and $M \in \mathrm{c} \mathcal{T}_{\omega}^{2}(R)$ by the diagram (4.17). It means that the assertion holds true for $n=2$. If $n \geqslant 3$, then the fact that $\operatorname{Im} f_{1} \in \mathrm{c} \mathcal{T}_{\omega}^{n-1}(R)$ implies $\theta_{\operatorname{Im} f_{1}}$ is an isomorphism. So $\operatorname{Tor}_{1}^{S}(\omega, N)=0$ by the diagram (4.16). In addition, we have $\left(\operatorname{Im} f_{1}\right)_{*} \in$ $\omega_{S}{ }^{\top n-3}$ by [32, Corollary 3.4(3)]. Because T-cograde ${ }_{\omega} \operatorname{Ext}_{R}^{n}\left(\omega, \operatorname{Ker} f_{n-1}\right) \geqslant n-1$ by assumption, applying the dimension shifting to (4.15) we obtain $M_{*} \in \omega_{S}{ }^{\top_{n-2}}$. Therefore we conclude that $M \in \mathrm{c} \mathcal{T}_{\omega}^{n}(R)$ by [32, Corollary 3.4(3)] again.

$(4) \Rightarrow(1)$ We proceed by induction on $n$. The case for $n=1$ is trivial. Let $M \in \Omega_{\mathcal{B}}^{n}(R)$ with $n \geqslant 2$ and let

$$
0 \rightarrow M \rightarrow B_{n-1} \rightarrow \cdots \rightarrow B_{1} \stackrel{f}{\longrightarrow} B_{0} \rightarrow L \rightarrow 0
$$

be an exact sequence with all $B_{i}$ in $\mathcal{B}_{\omega}(R)$. By (4), we have $L \in \mathrm{c} \mathcal{T}_{\omega}^{n}(R)$. By the induction hypothesis, we have T-cograde $\operatorname{Ext}_{R}^{i}(\omega, M) \geqslant i-1$ for any $1 \leqslant i \leqslant n-1$ and T-cograde ${ }_{\omega} \operatorname{Ext}_{R}^{n}(\omega, M) \geqslant n-2$.

Put $N:=\operatorname{cTr}_{\omega} \operatorname{Ker} f$. Because $B_{i} \in \mathrm{c} \mathcal{T}_{\omega}(R)$ by [32, Theorem 3.9], we obtain that $\omega \otimes_{S} N \cong L\left(\in \mathrm{c} \mathcal{T}_{\omega}^{n}(R)\right)$ and $N \in \omega_{S}{ }^{{ }^{n}{ }^{n-2}}$. By [33, Proposition 6.7], we have the following exact sequences

$$
\begin{gathered}
0 \rightarrow \operatorname{Ext}_{R}^{n-1}(\omega, M) \rightarrow N \stackrel{\pi}{\longrightarrow} \operatorname{Im} \mu_{N} \rightarrow 0, \\
0 \rightarrow \operatorname{Im} \mu_{N} \stackrel{\lambda}{\longrightarrow}\left(\omega \otimes_{S} N\right)_{*} \rightarrow \operatorname{Ext}_{R}^{n}(\omega, M) \rightarrow 0
\end{gathered}
$$

such that $\mu_{N}=\lambda \cdot \pi$. Since $\theta_{\omega \otimes_{S} N}$ is an isomorphism, it follows from [33, Lemma $6.1(2)]$ that $1_{\omega} \otimes \mu_{N}$ is also an isomorphism. Then both $1_{\omega} \otimes \lambda$ and $1_{\omega} \otimes \pi$ are isomorphisms.

From the exact sequence (4.18), we get $\operatorname{Im} \mu_{N} \in \omega_{S}{ }^{T_{n-2}}$. Because $\left(\omega \otimes_{S}\right.$ $N)_{*} \in \omega_{S}{ }^{T_{n-2}}$ by [32, Corollary 3.4], from the exact sequence (4.19) it yields that $\operatorname{Tor}_{n-2}^{S}\left(\omega, \operatorname{Ext}_{R}^{n}(\omega, M)\right)=0$. Thus we have T-cograde $\omega \operatorname{Ext}_{R}^{n}(\omega, M) \geqslant n-1$.

Lemma 4.18. For any $n \geqslant 0$, the following statements are equivalent.

(1) $\omega \otimes \operatorname{Ext}_{R}^{2}(\omega,-)$ vanishes on $\operatorname{Mod} R$.

(2) $\left(\operatorname{Tor}_{2}^{S}(\omega,-)\right)_{*}$ vanishes on $\operatorname{Mod} S$.

(3) $M_{*} \in \operatorname{ac} \mathcal{T}_{\omega}^{2}(S)$ for any $M \in \operatorname{Mod} R$.

(4) $\omega \otimes_{S} N \in \mathrm{c}^{2}{ }_{\omega}^{2}(R)$ for any $N \in \operatorname{Mod} S$.

Proof. By [35, Corollary 6.6], we have (3) $\Leftrightarrow(4)$. 
$(1) \Leftrightarrow(4)$ Assume that (1) holds true. Let $N \in \operatorname{Mod} S$. By [33, Lemma 6.1(2)], we have

$$
\theta_{\omega \otimes_{S} N} \cdot\left(1_{\omega} \otimes \mu_{N}\right)=1_{\omega \otimes_{S} N} .
$$

It follows that $\theta_{\omega \otimes_{S} N}$ is a split epimorphism and

$$
\begin{aligned}
& \operatorname{Ker} \theta_{\omega \otimes_{S} N} \cong \operatorname{Coker}\left(1_{\omega} \otimes \mu_{N}\right) \cong \omega \otimes_{S} \text { Coker } \mu_{N} \\
\cong & \left.\omega \otimes_{S} \operatorname{Ext}_{R}^{2}\left(\omega, \operatorname{acTr}_{\omega} N\right) \quad \text { (by [35, Corollary 5.2(2)] }\right) \\
= & 0 \quad(\text { by }(1)) .
\end{aligned}
$$

So $\theta_{\omega \otimes_{S} N}$ is a monomorphism, and hence an isomorphism.

Conversely, assume that (4) holds true. Let $M \in \operatorname{Mod} R$. By [33, Lemma 6.1(2)] again, we have

It follows that

$$
\theta_{\omega \otimes_{S} \mathrm{c} \operatorname{Tr}_{\omega} M} \cdot\left(1_{\omega} \otimes \mu_{\mathrm{c} \operatorname{Tr}_{\omega} M}\right)=1_{\omega \otimes_{S} \mathrm{c} \operatorname{Tr}_{\omega} M} .
$$

$$
\begin{aligned}
& \omega \otimes_{S} \operatorname{Ext}_{R}^{2}(\omega, M) \cong \omega \otimes_{S} \text { Coker } \mu_{\operatorname{cTr}_{\omega} M} \quad \text { (by [35, Corollary 5.3(2)]) } \\
\cong & \operatorname{Coker}\left(1_{\omega} \otimes \mu_{\mathrm{c} \operatorname{Tr}_{\omega} M} \cong \operatorname{Ker} \theta_{\omega \otimes_{S} \mathrm{c} \operatorname{Tr}_{\omega} M}\right. \\
= & 0 \quad(\text { by }(4)) .
\end{aligned}
$$

$(2) \Leftrightarrow(3)$ Assume that (2) holds true. Let $M \in \operatorname{Mod} R$. By [33, Lemma 6.1(1)], we have

$$
\left(\theta_{M}\right)_{*} \cdot \mu_{M_{*}}=1_{M_{*}} .
$$

It follows that $\mu_{M_{*}}$ is a split monomorphism and

$$
\begin{aligned}
& \operatorname{Coker} \mu_{M_{*}} \cong \operatorname{Ker}\left(\theta_{M}\right)_{*} \cong\left(\operatorname{Ker} \theta_{M}\right)_{*} \\
\cong & \left(\operatorname{Tor}_{2}^{S}\left(\omega, c \operatorname{Tr}_{\omega} M\right)\right)_{*} \quad(\text { by }[32, \text { Proposition 3.2] }) \\
= & 0 \quad(\text { by }(2)) .
\end{aligned}
$$

So $\mu_{M_{*}}$ is an epimorphism, and hence an isomorphism.

Conversely, assume that (3) holds true. Let $N \in \operatorname{Mod} S$. By [33, Lemma 6.1(1)] again, we have

It follows that

$$
\left(\theta_{\operatorname{acTr}_{\omega} N}\right)_{*} \cdot \mu_{\left(\operatorname{acTr}_{\omega} N\right)_{*}}=1_{\left(\operatorname{acTr}_{\omega} N\right)_{*}} \cdot
$$

$$
\begin{aligned}
& \left(\operatorname{Tor}_{2}^{S}(\omega, N)\right)_{*} \cong\left(\operatorname{Ker} \theta_{\left.\operatorname{acTr}_{\omega} N\right)_{*}} \quad(\text { by }[35, \text { Corollary 5.3(1)] })\right. \\
\cong & \operatorname{Ker}\left(\theta_{\operatorname{ac} \operatorname{Tr}_{\omega} N}\right)_{*} \cong \operatorname{Coker} \mu_{\left(\operatorname{ac} \operatorname{Tr}_{\omega} N\right)_{*}} \\
= & 0 \quad(\operatorname{by}(3)) .
\end{aligned}
$$

The following result establishes the left-right symmetry of certain cograde condition of modules.

Theorem 4.19. For any $n \geqslant 1$, the following statements are equivalent.

(1) $T$-cograde ${ }_{\omega} \operatorname{Ext}_{R}^{i}(\omega, M) \geqslant i-1$ for any $M \in \operatorname{Mod} R$ and $1 \leqslant i \leqslant n$.

(2) $E_{-}-\operatorname{cograde}_{\omega} \operatorname{Tor}_{i}^{S}(\omega, N) \geqslant i-1$ for any $N \in \operatorname{Mod} S$ and $1 \leqslant i \leqslant n$.

(3) $\cos \Omega^{i}(R) \subseteq \mathrm{c} \mathcal{T}_{\omega}^{i}(R)=\operatorname{co} \Omega_{\mathcal{B}}^{i}(R)$ for any $1 \leqslant i \leqslant n$.

(4) $\cos \Omega^{i}(R) \subseteq \mathrm{c} \mathcal{T}_{\omega}^{i}(R)=\operatorname{co} \Omega_{\mathcal{F}_{\omega}}^{i}(R)$ for any $1 \leqslant i \leqslant n$.

(5) $\cos \Omega^{i}(R) \subseteq \mathrm{c} \mathcal{T}_{\omega}^{i}(R)=\operatorname{co} \Omega_{\mathcal{P}_{\omega}}^{i}(R)$ for any $1 \leqslant i \leqslant n$.

(6) $\Omega_{\mathcal{F}}^{i}(S) \subseteq \operatorname{ac} \mathcal{T}_{\omega}^{i}(S)=\Omega_{\mathcal{A}}^{i}(S)$ for any $1 \leqslant i \leqslant n$.

(7) $\Omega_{\mathcal{F}}^{i}(S) \subseteq \operatorname{ac}_{\omega}^{i}(S)=\Omega_{\mathcal{I}_{\omega}}^{i}(S)$ for any $1 \leqslant i \leqslant n$. 
Proof. By Theorem 3.5 and Proposition 4.17, we have (1) $\Leftrightarrow(3) \Leftrightarrow(4) \Leftrightarrow(5)$. By Theorem 3.7 and Proposition 4.16, $(2) \Leftrightarrow(6) \Leftrightarrow(7)$.

In the following, we will prove (1) $\Leftrightarrow(2)$ by induction on $n$. The case for $n=1$ is trivial and the case for $n=2$ follows from Lemma 4.18, Now suppose $n \geqslant 3$.

(1) $\Rightarrow(2)$ Let $N \in \operatorname{Mod} S$. By the induction hypothesis, we have that E-cograde $_{\omega} \operatorname{Tor}_{i}^{S}(\omega, N) \geqslant i-1$ for any $1 \leqslant i \leqslant n-1$ and E-cograde $\operatorname{Tor}_{n}^{S}(\omega, N) \geqslant$ $n-2$. By Lemma 3.1(2), there exists an exact sequence

$$
0 \rightarrow \operatorname{Im}\left(f_{n} \otimes 1_{\omega}\right) \stackrel{\sigma}{\longrightarrow} \operatorname{ac}_{\omega} \Omega_{\mathcal{F}}^{n-1}(N) \stackrel{\tau}{\longrightarrow} \operatorname{Tor}_{n}^{S}(\omega, N) \rightarrow 0
$$

in $\operatorname{Mod} R$ such that $\sigma_{*}$ is an isomorphism. By Theorem 3.7, we have that $\Omega_{\mathcal{F}}^{n-1}(N) \in$ $\operatorname{ac} \mathcal{T}_{\omega}^{n-1}(S)$ and $\operatorname{ac} \operatorname{Tr}_{\omega} \Omega_{\mathcal{F}}^{n-1}(N) \in{ }_{R} \omega^{\perp_{n-1}}$. So

$$
\begin{gathered}
\operatorname{Ext}_{R}^{n-2}\left(\omega, \operatorname{Tor}_{n}^{S}(\omega, N)\right) \cong \operatorname{Ext}_{R}^{n-1}\left(\omega, \operatorname{Im}\left(f_{n} \otimes 1_{\omega}\right)\right) \\
\cong \operatorname{Ext}_{R}^{n}\left(\omega, \operatorname{acTr}_{\omega} \Omega_{\mathcal{F}}^{n}(N)\right) .
\end{gathered}
$$

Then T-cograde $\operatorname{Ext}_{R}^{n-2}\left(\omega, \operatorname{Tor}_{n}^{S}(\omega, N)\right) \geqslant n-1$ by (1). It follows from Lemma4.11(1) that E-cograde $\operatorname{Tor}_{n}^{S}(\omega, N) \geqslant n-1$.

(2) $\Rightarrow$ (1) Let $M \in \operatorname{Mod} R$. By the induction hypothesis, we have that T-cograde $\operatorname{Ext}_{R}^{i}(\omega, M) \geqslant i-1$ for any $1 \leqslant i \leqslant n-1$ and T-cograde ${ }_{\omega} \operatorname{Ext}_{R}^{n}(\omega, M) \geqslant$ $n-2$. By Lemma 3.1(1), there exists an exact sequence

$$
0 \rightarrow \operatorname{Ext}_{R}^{n+1}(\omega, M) \stackrel{\lambda}{\longrightarrow} \operatorname{cTr}_{\omega} \cos ^{n}(M) \stackrel{\pi}{\longrightarrow} I^{n+1}(M)_{*} / \cos ^{n+1}(M)_{*} \rightarrow 0
$$

in Mod $S$ such that $1_{\omega} \otimes \pi$ is an isomorphism. By Theorem 3.5, we have that $\cos \Omega^{n-1}(M) \in \mathrm{c} \mathcal{T}_{\omega}^{n-1}(R)$ and $\operatorname{cTr}_{\omega} \operatorname{co} \Omega^{n-1}(M) \in \omega_{S}{ }^{T^{n-1}}$. So

$$
\begin{gathered}
\operatorname{Tor}_{n-2}^{S}\left(\omega, \operatorname{Ext}_{R}^{n}(\omega, M)\right) \cong \operatorname{Tor}_{n-1}^{S}\left(\omega, I^{n}(M)_{*} / \operatorname{co}^{n}(M)_{*}\right) \\
\cong \operatorname{Tor}_{n}^{S}\left(\omega, \operatorname{cTr}_{\omega} \operatorname{co} \Omega^{n}(M)\right) .
\end{gathered}
$$

Then E-cograde ${ }_{\omega} \operatorname{Tor}_{n-2}^{S}\left(\omega, \operatorname{Ext}_{R}^{n}(\omega, M)\right) \geqslant n-1$ by (2). It follows from Lemma4.11(2) that T-cograde $\omega_{\omega} \operatorname{Ext}_{R}^{n}(\omega, M) \geqslant n-1$.

\subsection{Examples}

In this subsection, we give some examples for $\omega$ satisfying the (quasi) $n$-cograde condition.

Let $R$ be an artin algebra. Recall that $R$ is called Auslander $n$-Gorenstein if $\operatorname{pd}_{R^{\text {op }}} I^{i}\left(R_{R}\right) \leqslant i$ for any $0 \leqslant i \leqslant n-1$; equivalently $\operatorname{pd}_{R} I^{i}\left({ }_{R} R\right) \leqslant i$ for any $0 \leqslant i \leqslant n-1([16,26])$; and $R$ is called left (resp. right) quasi $n$-Gorenstein if $\operatorname{pd}_{R} I^{i}\left({ }_{R} R\right)\left(\operatorname{resp} . \operatorname{pd}_{R^{o p}} I^{i}\left(R_{R}\right) \leqslant i+1\right.$ for any $0 \leqslant i \leqslant n-1([22])$.

Let $D$ be the ordinary duality between $\bmod R$ and $\bmod R^{o p}$. Then $D(R)$ is a semidualizing $(R, R)$-bimodule. Because

$$
\begin{gathered}
\operatorname{pd}_{R} I^{i}\left({ }_{R} R\right)=\operatorname{id}_{R^{o p}} P_{i}\left(D\left({ }_{R} R\right)\right)=\operatorname{pd}_{R} \operatorname{Hom}_{R^{o p}}\left(P_{i}\left(D\left({ }_{R} R\right)\right), D(R)\right) \text { and } \\
\operatorname{pd}_{R^{o p}} I^{i}\left(R_{R}\right)=\operatorname{id}_{R} P_{i}\left(D\left(R_{R}\right)\right)=\operatorname{pd}_{R^{o p}} \operatorname{Hom}_{R}\left(P_{i}\left(D\left(R_{R}\right)\right), D(R)\right),
\end{gathered}
$$

we have

\section{Example 4.20.}

(1) $R$ is Auslander $n$-Gorenstein if and only if $D(R)$ satisfies the $n$-cograde condition.

(2) $R$ is left (resp. right) quasi $n$-Gorenstein if and only if $D(R)$ satisfies the left (resp. right) quasi $n$-cograde condition. 
So, if putting ${ }_{R} \omega_{S}={ }_{R} D(R)_{R}$ in Theorem 4.8 (resp. Theorem4.14), then all the conditions there are equivalent to that $R$ is Auslander $n$-Gorenstein (resp. right quasi $n$-Gorenstein). Note that the notion of quasi $n$-Gorenstein algebras is not left-right symmetric ([5, p.11]). So, contrary to the $n$-cograde condition, the quasi $n$-cograde condition is not left-right symmetric.

Example 4.21. Let $Q$ be the quiver

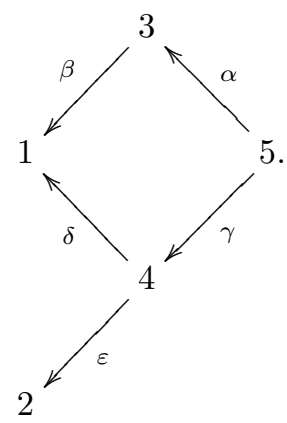

and $R=K Q /<\beta \alpha-\delta \gamma, \varepsilon \gamma>$ with $K$ a field. Take

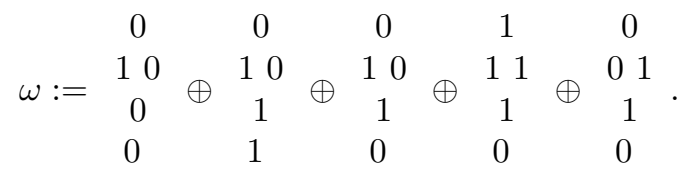

By [3. Example VI.2.8(a)], we have that $\omega_{R}$ is a non-injective tilting module with $\operatorname{pd}_{R} \omega=1$. Thus it is a semidualizing $\left(R, \operatorname{End}_{R}(\omega)\right)$-bimodule. It is straightforward to verify that the projective cover $P_{0}(\omega)$ of $\omega$ is $P(1) \oplus P(4)^{2} \oplus P(5)^{2}$. So $\mathcal{P}_{\omega}(R)$ $\operatorname{id}_{R} P_{0}(\omega)=0$, and hence $\omega$ satisfies the left and right 1-cograde conditions by Theorem 4.8. Since $\operatorname{pd}_{R} \omega=1$, we have $\operatorname{Ext}_{R} \geqslant 2(\omega, M)=0$ for any $M \in \operatorname{Mod} R$. By Theorem 4.8 again, we have that $\omega$ satisfies the left and right $n$-cograde conditions for any $n \geqslant 1$.

\section{Two cotorsion pairs}

In this section, we will construct two complete cotorsion pairs under any of the equivalent conditions in Theorem 4.19.

For any $n \geqslant 0$, set $\mathcal{P}_{\omega^{-}} \mathrm{id}^{\leqslant n}(R):=\left\{M \in \operatorname{Mod} R \mid \mathcal{P}_{\omega}(R)-\operatorname{id}_{R} M \leqslant n\right\}$.

Lemma 5.1. Let $M \in{ }_{R} \omega^{\perp_{n-1}}$ with $n \geqslant 1$. If $\mathrm{T}-\operatorname{cograde}_{\omega} \operatorname{Ext}_{R}^{n}(\omega, M) \geqslant n-1$, then there exists an exact sequence

$$
0 \rightarrow M \rightarrow X \rightarrow Y \rightarrow 0
$$

in $\operatorname{Mod} R$ with $X \in{ }_{R} \omega^{\perp_{n}}$ and $Y \in \mathcal{P}_{\omega}-\mathrm{id}^{\leqslant n-1}(R)$.

Proof. Let $M \in{ }_{R} \omega^{\perp_{n-1}}$. From the exact sequence

$$
0 \rightarrow M \rightarrow I^{0}(M) \rightarrow \cdots \rightarrow I^{n-1}(M) \rightarrow \cos ^{n}(M) \rightarrow 0
$$


in $\operatorname{Mod} R$, we get the following commutative diagram with exact rows

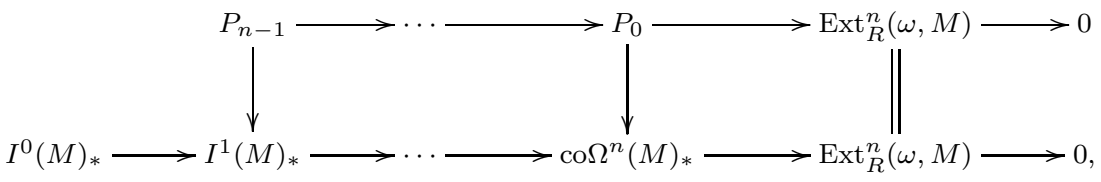

Diagram (5.1)

where the upper sequence is a projective resolution of $\operatorname{Ext}_{R}^{n}(\omega, M)$ in $\operatorname{Mod} S$. Taking the mapping cone of the diagram (5.1), we get an exact sequence

$$
I^{0}(M)_{*} \oplus P_{n-1} \rightarrow \cdots \rightarrow I^{n-1}(M)_{*} \oplus P_{0} \rightarrow \operatorname{co} \Omega^{n}(M)_{*} \rightarrow 0 .
$$

Since T-cograde ${ }_{\omega} \operatorname{Ext}_{R}^{n}(\omega, M) \geqslant n-1$, we get an exact sequence

$$
\omega \otimes_{S} P_{n-1} \rightarrow \cdots \rightarrow \omega \otimes_{S} P_{1} \rightarrow \omega \otimes_{S} P_{0} \rightarrow 0
$$

in $\operatorname{Mod} R$. Then we get the following commutative diagram with exact columns and rows

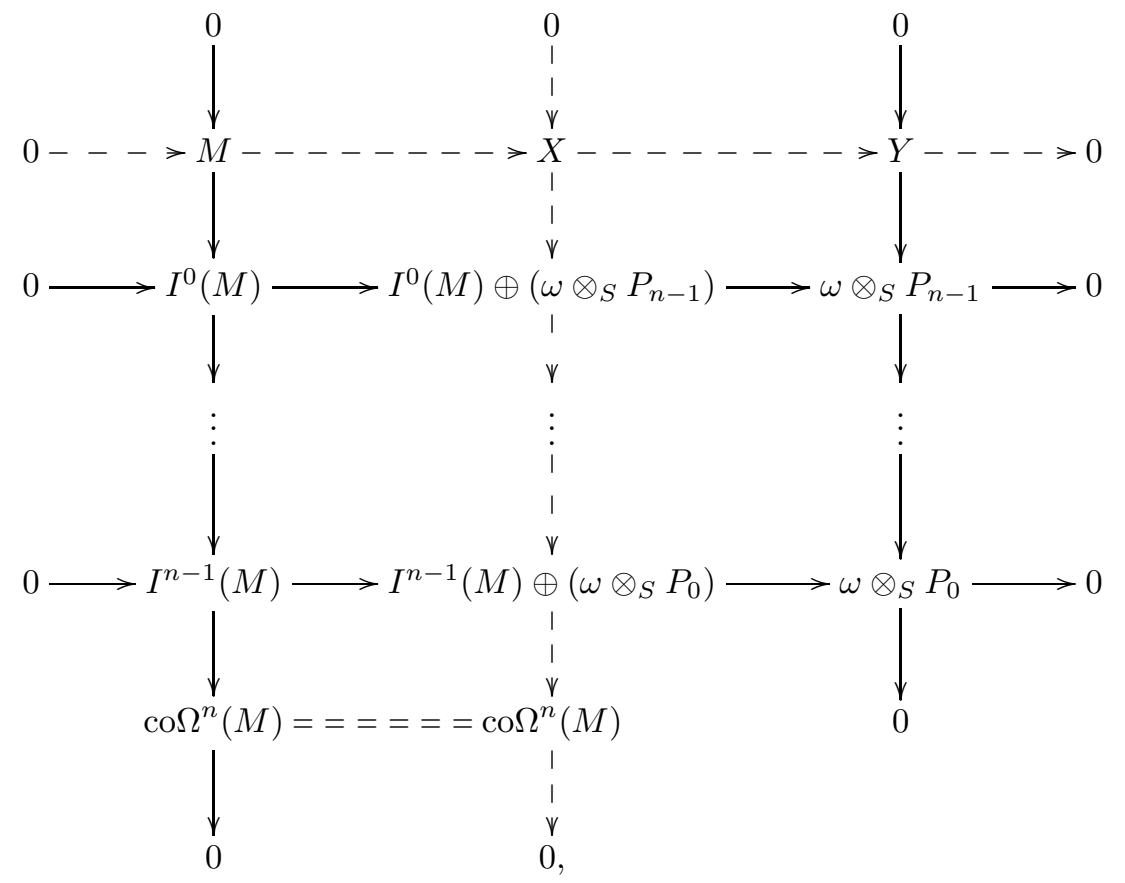

Diagram (5.3)

where

$$
\begin{gathered}
X=\operatorname{Ker}\left(I^{0}(M) \oplus\left(\omega \otimes_{S} P_{n-1}\right) \rightarrow I^{1}(M) \oplus\left(\omega \otimes_{S} P_{n-2}\right)\right) \text { and } \\
Y=\operatorname{Ker}\left(\omega \otimes_{S} P_{n-1} \rightarrow \omega \otimes_{S} P_{n-2}\right) .
\end{gathered}
$$


Then $Y \in \mathcal{P}_{\omega^{-}} \mathrm{id}^{\leqslant n-1}(R)$. From the exactness of (5.2) and the middle column in the diagram (5.3), we know that $X \in{ }_{R} \omega^{\perp_{n}}$. So the top row in the diagram (5.3) is the desired exact sequence.

For any $n \geqslant 0$, set $\mathcal{I}_{\omega}-\operatorname{pd}^{\leqslant n}(S):=\left\{N \in \operatorname{Mod} S \mid \mathcal{I}_{\omega}(S)-\operatorname{pd}_{S} N \leqslant n\right\}$.

Lemma 5.2. Let $N \in \omega_{S}^{\top{ }_{n-1}}$ with $n \geqslant 1$. If E-cograde ${ }_{\omega} \operatorname{Tor}_{n}^{S}(\omega, N) \geqslant n-1$, then there exists an exact sequence

$$
0 \rightarrow Y^{\prime} \rightarrow X^{\prime} \rightarrow N \rightarrow 0
$$

in $\operatorname{Mod} S$ with $X^{\prime} \in \omega_{S}^{\top n}$ and $Y^{\prime} \in \mathcal{I}_{\omega}-\mathrm{pd}^{\leqslant n-1}(S)$.

Proof. Let $N \in \omega_{S}^{\top}{ }^{\top} n-1$. From the exact sequence

$$
0 \rightarrow \Omega_{\mathcal{F}}^{n}(N) \rightarrow F_{n-1}(N) \rightarrow \cdots \rightarrow F_{0}(N) \rightarrow N \rightarrow 0
$$

in $\operatorname{Mod} S$, we get the following commutative diagram with exact rows

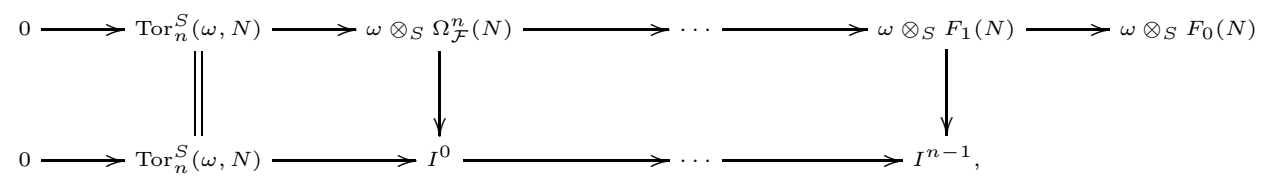

Diagram (5.4)

where the lower sequence is an injective resolution of $\operatorname{Tor}_{n}^{S}(\omega, N)$ in $\operatorname{Mod} R$. Taking the mapping cone of diagram (5.4), we get an exact sequence

$$
\omega \otimes_{S} \Omega_{\mathcal{F}}^{n}(N) \rightarrow I^{0} \oplus\left(\omega \otimes_{S} F_{n-1}(N)\right) \rightarrow \cdots \rightarrow I^{n-1} \oplus\left(\omega \otimes_{S} F_{0}(N)\right)
$$

Since E-cograde ${ }_{\omega} \operatorname{Tor}_{n}^{S}(\omega, N) \geqslant n-1$, we get an exact sequence

$$
0 \rightarrow I_{*}^{0} \rightarrow I_{*}^{1} \rightarrow \cdots \rightarrow I_{*}^{n-1}
$$


in Mod $S$. Then we get the following commutative diagram with exact columns and rows

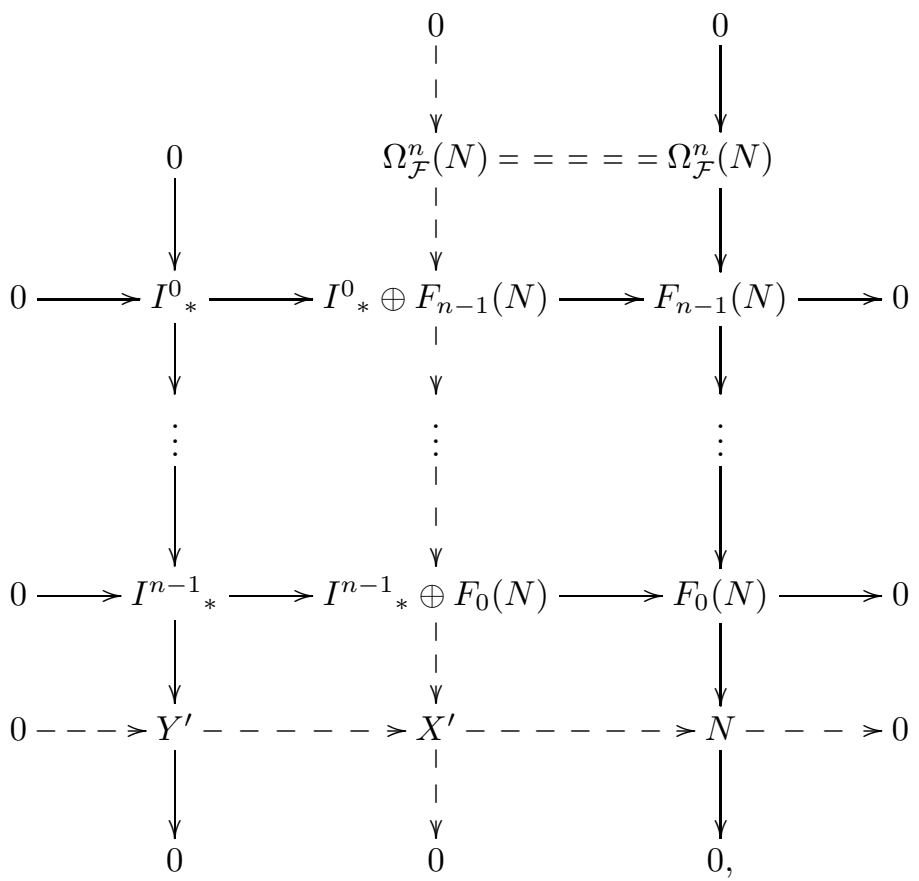

Diagram (5.6)

where

$$
\begin{gathered}
X^{\prime}=\operatorname{Coker}\left(I^{n-2}{ }_{*} \oplus F_{1}(N) \rightarrow I^{n-1}{ }_{*} \oplus F_{0}(N)\right) \text { and } \\
Y^{\prime}=\operatorname{Coker}\left(I^{n-2}{ }_{*} \rightarrow I^{n-1}{ }_{*}\right) .
\end{gathered}
$$

Then $Y^{\prime} \in \mathcal{I}_{\omega^{-}} \mathrm{pd}^{\leqslant n-1}(S)$. From the exactness of (5.5) and the middle column in the diagram (5.6), we know that $X^{\prime} \in \omega_{S}{ }^{{ }_{n}}$. So the bottom row in the diagram (5.6) is the desired exact sequence.

Lemma 5.3. For any $n \geqslant 0$, we have

(1) $\mathcal{P}_{\omega}$-id ${ }^{\leqslant n}(R)$ is closed under direct summands and closed under extensions.

(2) $\mathcal{I}_{\omega}-\mathrm{pd}^{\leqslant n}(S)$ is closed under direct summands and closed under extensions.

Proof. (1) By [33, Lemma 4.6], $\mathcal{P}_{\omega}$ - id ${ }^{\leqslant n}(R)$ is closed under direct summands.

Let

$$
0 \rightarrow A \rightarrow B \rightarrow C \rightarrow 0
$$

be an exact sequence in $\operatorname{Mod} R$ with $A, C \in \mathcal{P}_{\omega^{-}} \mathrm{id}^{\leqslant n}(R)$. It is easy to see that it is $\operatorname{Hom}_{R}\left(-, \mathcal{P}_{\omega}(R)\right)$-exact. Then $B \in \mathcal{P}_{\omega}$ - id ${ }^{\leqslant n}(R)$ by the generalized horseshoe lemma (c.f. [23, Lemma 3.1(2)]).

(2) By [34, Lemma 4.7], $\mathcal{I}_{\omega}$ - $\mathrm{pd}^{\leqslant n}(S)$ is closed under direct summands.

Let

$$
0 \rightarrow A \rightarrow B \rightarrow C \rightarrow 0
$$

be an exact sequence in $\operatorname{Mod} S$ with $A, C \in \mathcal{I}_{\omega}$ - pd ${ }^{\leqslant n}(S)$. It is easy to see that it is $\left(\omega \otimes_{S}-\right)$-exact; equivalently it is $\operatorname{Hom}_{R}\left(-, \mathcal{I}_{\omega}(S)\right)$-exact by [34, p.298, Observation]. 
Then $B \in \mathcal{I}_{\omega-\mathrm{pd}} \leqslant n(S)$ by the generalized horseshoe lemma (c.f. 23, Lemma $3.1(1)])$.

Proposition 5.4. Let $n, k \geqslant 1$ and $\mathrm{T}$-cograde ${ }_{\omega} \operatorname{Ext}_{R}^{i+k}(\omega, M) \geqslant i$ for any $M \in$ $\operatorname{Mod} R$ and $1 \leqslant i \leqslant n-1$. Then for any $M \in \operatorname{Mod} R$ and $0 \leqslant i \leqslant n-1$, there exists an exact sequence

$$
0 \rightarrow \operatorname{co} \Omega^{k-1}(M) \rightarrow X \rightarrow Y \rightarrow 0
$$

in $\operatorname{Mod} R$ with $X \in{ }_{R} \omega^{\perp_{i+1}}$ and $Y \in \mathcal{P}_{\omega}-\mathrm{id}^{\leqslant i}(R)$.

Proof. We proceed by induction on $n$. The case for $n=1$ follows from Lemma 5.1 Now suppose $n \geqslant 2$. By the induction hypothesis, for any $0 \leqslant i \leqslant n-2$ there exists an exact sequence

$$
0 \rightarrow \operatorname{co} \Omega^{k-1}(M) \rightarrow X_{i} \rightarrow Y_{i} \rightarrow 0
$$

in $\operatorname{Mod} R$ with $X_{i} \in{ }_{R} \omega^{\perp_{i+1}}$ and $Y_{i} \in \mathcal{P}_{\omega}$ - id ${ }^{\leqslant i}(R)$. Then

$$
\operatorname{Ext}_{R}^{n}\left(\omega, X_{n-2}\right) \cong \operatorname{Ext}_{R}^{n}\left(\omega, \operatorname{co}^{k-1}(M)\right) \cong \operatorname{Ext}_{R}^{n+k-1}(\omega, M) .
$$

So T-cograde ${ }_{\omega} \operatorname{Ext}_{R}^{n}\left(\omega, X_{n-2}\right)=$ T-cograde ${ }_{\omega} \operatorname{Ext}_{R}^{n+k-1}(\omega, M) \geqslant n-1$ by assumption. Applying Lemma 5.1, we get an exact sequence

$$
0 \rightarrow X_{n-2} \rightarrow X_{n-1} \rightarrow Y_{n-1} \rightarrow 0
$$

in $\operatorname{Mod} R$ with $X_{n-1} \in{ }_{R} \omega^{\perp_{n}}$ and $Y_{n-1} \in \mathcal{P}_{\omega^{-}}$id $^{\leqslant n-1}(R)$. Consider the following push-out diagram

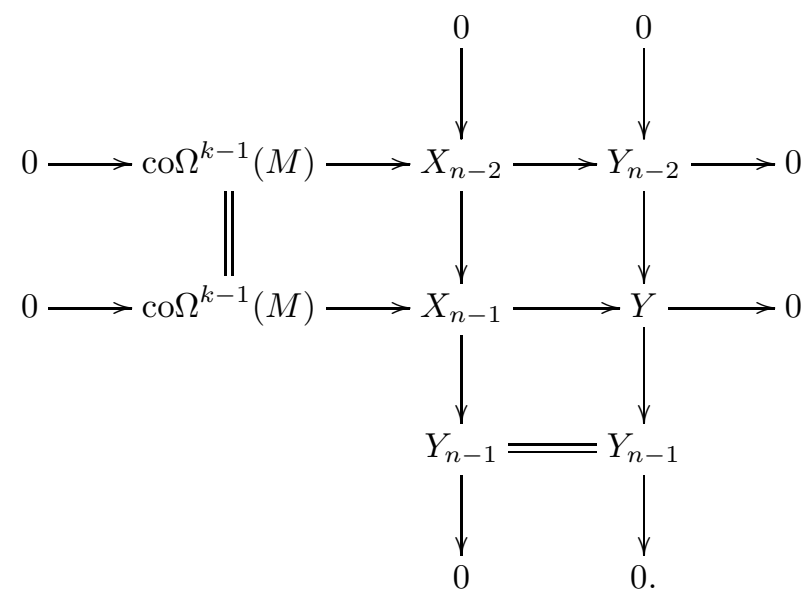

By Lemma 5.3(1), we have $Y \in \mathcal{P}_{\omega^{-}} \mathrm{id}^{\leqslant n-1}(R)$. So the middle row in this diagram is the desired sequence.

Proposition 5.5. Let $n, k \geqslant 1$ and E-cograde ${ }_{\omega} \operatorname{Tor}_{i+k}^{S}(\omega, N) \geqslant i$ for any $N \in$ $\operatorname{Mod} S$ and $1 \leqslant i \leqslant n-1$. Then for any $N \in \operatorname{Mod} S$ and $0 \leqslant i \leqslant n-1$, there exists an exact sequence

$$
0 \rightarrow Y^{\prime} \rightarrow X^{\prime} \rightarrow \Omega_{\mathcal{F}}^{k-1}(N) \rightarrow 0
$$

in $\operatorname{Mod} S$ with $X^{\prime} \in \omega_{S}{ }^{\top i+1}$ and $Y^{\prime} \in \mathcal{I}_{\omega}-\mathrm{pd}^{\leqslant i}(S)$. 
Proof. We proceed by induction on $n$. The case for $n=1$ follows from Lemma 5.2 Now suppose $n \geqslant 2$. By the induction hypothesis, for any $0 \leqslant i \leqslant n-2$ there exists an exact sequence

$$
0 \rightarrow Y_{i}^{\prime} \rightarrow X_{i}^{\prime} \rightarrow \Omega_{\mathcal{F}}^{k-1}(N) \rightarrow 0
$$

in $\operatorname{Mod} S$ with $X_{i}^{\prime} \in \omega_{S}^{\top i+1}$ and $Y_{i}^{\prime} \in \mathcal{I}_{\omega^{-}} \operatorname{pd}^{\leqslant i}(S)$. Then

$$
\operatorname{Tor}_{n}^{S}\left(\omega, X_{n-2}^{\prime}\right) \cong \operatorname{Tor}_{n}^{S}\left(\omega, \Omega_{\mathcal{F}}^{k-1}(N)\right) \cong \operatorname{Tor}_{n+k-1}^{S}(\omega, N) .
$$

So E-cograde ${ }_{\omega} \operatorname{Tor}_{n}^{S}\left(\omega, X_{n-2}^{\prime}\right)=$ E-cograde $_{\omega} \operatorname{Tor}_{n+k-1}^{S}(\omega, N) \geqslant n-1$ by assumption. Applying Lemma 5.2, we get an exact sequence

$$
0 \rightarrow Y_{n-1}^{\prime} \rightarrow X_{n-1}^{\prime} \rightarrow X_{n-2}^{\prime} \rightarrow 0
$$

in Mod $S$ with $X_{n-1}^{\prime} \in \omega_{S}{ }^{\top_{n}}$ and $Y_{n-1}^{\prime} \in \mathcal{I}_{\omega}-\operatorname{pd}^{n-1}(S)$. Consider the following pull-back diagram

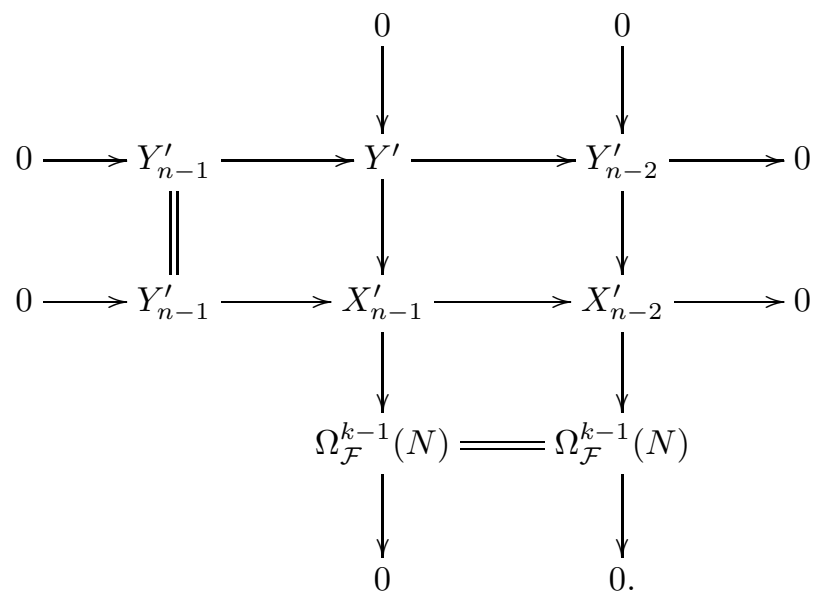

By Lemma 5.3(2), we have $Y^{\prime} \in \mathcal{I}_{\omega^{-}} \mathrm{pd}^{\leqslant n-1}(S)$. So the middle column in this diagram is the desired sequence.

Based on the equivalence of (1) and (2) in Theorem 4.19, we have the following

Theorem 5.6. For any $n \geqslant 1$, we have

(1) If one of the equivalent conditions in Theorem 4.19 is satisfied, then the following statements are equivalent.

(1.1) $\operatorname{pd}_{S^{o p}} \omega \leqslant n-1$.

(1.2) $\mathcal{P}_{\omega}(R)-\operatorname{id}_{R} R \leqslant n-1$.

(1.3) $\mathcal{P}_{\omega}(R)-\operatorname{id}_{R} P \leqslant n-1$ for any projective $P$ in $\operatorname{Mod} R$.

(1.4) $\left(\mathcal{P}_{\omega}-\mathrm{id}^{\leqslant n-1}(R),{ }_{R} \omega^{\perp_{n}}\right)$ forms a complete cotorsion pair.

(2) If one of the equivalent conditions in Theorem 4.19 is satisfied, then the following statements are equivalent.

(2.1) $\mathcal{I}_{\omega}(S)-\operatorname{pd}_{S} Q \leqslant n-1$ for some injective cogenerator $Q$ in $\operatorname{Mod} S$.

(2.2) $\mathcal{I}_{\omega}(S)-\operatorname{pd}_{S} I \leqslant n-1$ for any injective module $I$ in $\operatorname{Mod} S$.

(2.3) $\left(\omega_{S}{ }^{T_{n}}, \mathcal{I}_{\omega}-\mathrm{pd}^{\leqslant n-1}(S)\right)$ forms a complete cotorsion pair.

If $R$ and $S$ are artin algebras, then the statements (2.1)-(2.3) are equivalent to the following

(2.4) $\operatorname{pd}_{R} \omega \leqslant n-1$. 
Proof. By Lemma 4.5(1), we have $(1.1) \Leftrightarrow(1.2)$.

If $\mathcal{P}_{\omega}(R)-\mathrm{id}_{R} R \leqslant n-1$, then $\mathcal{P}_{\omega}(R)-\mathrm{id}_{R} F \leqslant n-1$ for any free module $F$ in $\operatorname{Mod} R$ by [19, Proposition 5.1(b)]. It follows from Lemma 5.3(1) that $\mathcal{P}_{\omega}(R)-$ $\operatorname{id}_{R} P \leqslant n-1$ for any projective $P$ in $\operatorname{Mod} R$. This proves $(1.2) \Leftrightarrow(1.3)$.

$(1.3) \Rightarrow(1.4)$ It is easy to verify that $\operatorname{Ext}_{R}^{1}(A, B)=0$ for any $A \in \mathcal{P}_{\omega^{-}} \mathrm{id}^{\leqslant n-1}(R)$ and $B \in R_{R} \omega^{\perp_{n}}$.

Let $M \in \operatorname{Mod} R$. By Lemma 5.1 when $n=1$ or taking $k=1$ in Proposition 5.4 when $n \geqslant 2$, we get an exact sequence

$$
0 \rightarrow M \rightarrow B \rightarrow A \rightarrow 0
$$

in $\operatorname{Mod} R$ with $B \in R_{R} \omega^{\perp_{n}}$ and $A \in \mathcal{P}_{\omega^{-}} \mathrm{id}^{\leqslant n-1}(R)$. It implies that $M$ has a special ${ }_{R} \omega^{\perp_{n}}$-preenvelope and $R_{R} \omega^{\perp_{n}}$ is special preenveloping in $\operatorname{Mod} R$. If $M \in$ $\left(\mathcal{P}_{\omega} \text {-id }{ }^{\leqslant n-1}(R)\right)^{\perp_{1}}$, then the exact sequence $(5.7)$ splits. It follows that $M$ is a direct summand of $B$ and $M \in{ }_{R} \omega^{\perp_{n}}$.

Let

$$
0 \rightarrow M_{1} \rightarrow P \rightarrow M \rightarrow 0
$$

be an exact sequence in $\operatorname{Mod} R$ with $P$ projective. By (1.3), we have $P \in \mathcal{P}_{\omega}$-id ${ }^{\leqslant n-1}(R)$. By Lemma 5.1 when $n=1$ or by Proposition 5.4 when $n \geqslant 2$, we have an exact sequence

$$
0 \rightarrow M_{1} \rightarrow B^{\prime} \rightarrow A^{\prime} \rightarrow 0
$$

in $\operatorname{Mod} R$ with $B^{\prime} \in{ }_{R} \omega^{\perp_{n}}$ and $A^{\prime} \in \mathcal{P}_{\omega^{-}}$id ${ }^{\leqslant n-1}(R)$. Consider the following pushout diagram

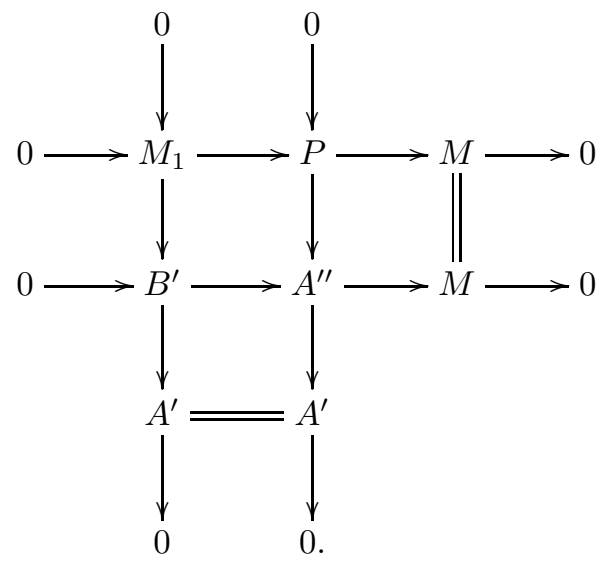

Since $\mathcal{P}_{\omega^{-}}$id ${ }^{\leqslant n-1}(R)$ is closed under extensions by Lemma 5.3 (1), it follows from the middle column in the above diagram that $A^{\prime \prime} \in \mathcal{P}_{\omega^{-}}$id ${ }^{\leqslant n-1}(R)$. If $M \in{ }^{\perp_{1}}\left({ }_{R} \omega^{\perp_{n}}\right)$, then the middle row in the above diagram splits and $M$ is a direct summand of $A^{\prime \prime}$. By Lemma $5.3(1)$, we have $M \in \mathcal{P}_{\omega^{-}} \mathrm{id}^{\leqslant n-1}(R)$. It follows from Lemma 2.7 that $\left(\mathcal{P}_{\omega^{-}} \mathrm{id}^{\leqslant n-1}(R), R_{R} \omega^{\perp_{n}}\right)$ forms a complete cotorsion pair.

$(1.4) \Rightarrow(1.2)$ By (1.4), we immediately have that ${ }_{R} R \in \mathcal{P}_{\omega^{-}}$id ${ }^{\leqslant n-1}(R)$ and $\mathcal{P}_{\omega}(R)-\mathrm{id}_{R} R \leqslant n-1$.

If $\mathcal{I}_{\omega}(S)-\operatorname{pd}_{S} Q \leqslant n-1$ for some injective cogenerator $Q$ in $\operatorname{Mod} S$, then any direct product of $Q$ is in $\mathcal{I}_{\omega}$ - $\mathrm{pd}^{\leqslant n-1}(S)$ by [19, Proposition 5.1(c)]. It follows from Lemma [5.3 (2) that $\mathcal{I}_{\omega}(S)-\operatorname{pd}_{S} I \leqslant n-1$ for any injective module $I$ in $\operatorname{Mod} S$. This proves $(2.1) \Leftrightarrow(2.2)$. 
$(2.2) \Rightarrow(2.3)$ It is easy to verify that $\operatorname{Ext}_{S}^{1}(C, D)=0$ for any $C \in \omega_{S}{ }^{T_{n}}$ and $D \in \mathcal{I}_{\omega^{-}} \mathrm{pd}^{\leqslant n-1}(S)$.

Let $N \in \operatorname{Mod} S$. By Lemma 5.2 when $n=1$ or taking $k=1$ in Proposition 5.5 when $n \geqslant 2$, we get an exact sequence

$$
0 \rightarrow D \rightarrow C \rightarrow N \rightarrow 0
$$

in $\operatorname{Mod} S$ with $C \in \omega_{S}{ }^{\top n}$ and $D \in \mathcal{I}_{\omega}$ - $d^{\leqslant n-1}(S)$. It implies that $N$ has a special $\omega_{S}{ }^{\top n}$-precover and $\omega_{S}^{\top_{n}}$ is precovering in $\operatorname{Mod} S$. If $N \in{ }^{\perp_{1}}\left(\mathcal{I}_{\omega^{-}} \operatorname{pd}^{\leqslant n-1}(S)\right)$, then the exact sequence (5.8) splits. It follows that $N$ is a direct summand of $C$ and $N \in \omega_{S}{ }^{T_{n}}$.

Let

$$
0 \rightarrow N \rightarrow I \rightarrow N_{1} \rightarrow 0
$$

be an exact sequence in Mod $S$ with $I$ injective. By (2.2), we have $I \in \mathcal{I}_{\omega^{-}} \operatorname{pd}^{\leqslant n-1}(S)$. By Lemma 5.2 when $n=1$ or by Proposition 5.5 when $n \geqslant 2$, we have an exact sequence

$$
0 \rightarrow D^{\prime} \rightarrow C^{\prime} \rightarrow N_{1} \rightarrow 0
$$

in Mod $S$ with $C^{\prime} \in \omega_{S}{ }^{\top n}$ and $D^{\prime} \in \mathcal{I}_{\omega}$ - $\operatorname{pd}^{\leqslant n-1}(S)$. Consider the following pullback diagram

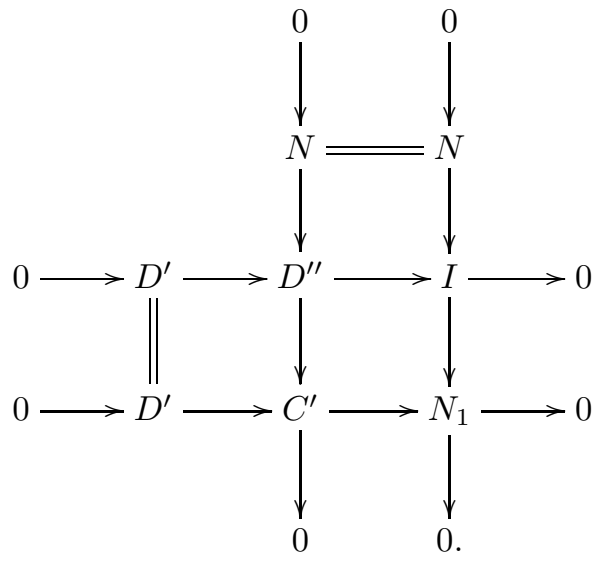

Since $\mathcal{I}_{\omega}$ - $\mathrm{pd}^{\leqslant n-1}(S)$ is closed under extensions by Lemma 5.3 (2), it follows from the middle row in the above diagram that $D^{\prime \prime} \in \mathcal{I}_{\omega}$ - $\mathrm{pd}^{\leqslant n-1}(S)$. If $N \in\left(\omega_{S}{ }^{\top n}\right)^{\perp_{1}}$, then the middle column in the above diagram splits and $N$ is a direct summand of $D^{\prime \prime}$. By Lemma $5.3(2)$, we have $N \in \mathcal{I}_{\omega}$ - $\mathrm{pd}^{\leqslant n-1}(S)$. It follows from Lemma 2.7 that $\left(\omega_{S}{ }^{\top n}, \mathcal{I}_{\omega^{-}} \mathrm{pd}^{\leqslant n-1}(S)\right)$ forms a complete cotorsion pair.

$(2.3) \Rightarrow(2.2)$ For any injective module $I$ in $\operatorname{Mod} S$, by (2.3) we have that $I \in$ $\mathcal{I}_{\omega}-\mathrm{pd}^{\leqslant n-1}(S)$ and $\mathcal{I}_{\omega}(S)-\operatorname{pd}_{S} I \leqslant n-1$.

If $R$ and $S$ are artin algebras, then $\operatorname{pd}_{R} \omega=\mathcal{I}_{\omega}(S)-\operatorname{pd}_{S} D\left(S_{S}\right)$ by 34, Lemma 4.9]. Because $D\left(S_{S}\right)$ is an injective cogenerator in $\operatorname{Mod} S,(2.1) \Leftrightarrow(2.4)$ follows.

Observation 5.7. Let $R$ be an artin algebra and ${ }_{R} \omega_{S}={ }_{R} D(R)_{R}$. Then we have

(1) $\operatorname{pd}_{R} \omega=\operatorname{id}_{R^{o p}} R$ and $\operatorname{pd}_{R^{o p}} \omega=\operatorname{id}_{R} R$.

(2) $\mathcal{P}_{\omega}(R)$ is exactly the subclass of $\operatorname{Mod} R$ consisting of injective modules. It implies that

(2.1) $\mathcal{P}_{\omega}(R)-\operatorname{id}_{R} M=\operatorname{id}_{R} M$ for any $M \in \operatorname{Mod} R$.

(2.2) $\mathcal{P}_{\omega^{-}} \mathrm{id}^{\leqslant n}(R)=\mathcal{I}^{\leqslant n}(R):=\left\{M \in \operatorname{Mod} R \mid \operatorname{id}_{R} M \leqslant n\right\}$. 
(3) $\mathcal{I}_{\omega}(R)$ is exactly the subclass of $\operatorname{Mod} R$ consisting of projective modules. It implies that

(3.1) $\mathcal{I}_{\omega}(R)-\operatorname{pd}_{R} N=\operatorname{pd}_{R} N$ for any $N \in \operatorname{Mod} R$.

(3.2) $\mathcal{I}_{\omega}-\mathrm{pd}^{\leqslant n}(R)=\mathcal{P} \leqslant n(R):=\left\{N \in \operatorname{Mod} R \mid \operatorname{pd}_{R} N \leqslant n\right\}$.

(4) By [11, Proposition VI.5.3], it is easy to see that $\omega_{R}{ }^{\top}{ }_{n+1}={ }^{{ }_{n+1}} R R$.

(5) If $R$ is right quasi $(n-1)$-Gorenstein, then all conditions in Theorem 4.19 are satisfied; see Theorem 4.14 and Example 4.20(2).

As an application of Theorem 5.6, we have the following

Corollary 5.8. Let $R$ be a right quasi $(n-1)$-Gorenstein artin algebra with $n \geqslant 1$. Then the following statements are equivalent.

(1) $\operatorname{id}_{R} R \leqslant n-1$.

(2) $\operatorname{id}_{R^{o p}} R \leqslant n-1$.

(3) $\left(\mathcal{I}^{\leqslant n-1}(R),{ }_{R} D(R)^{\perp_{n}}\right)$ forms a complete cotorsion pair.

(4) $\left({ }^{\perp_{n}} R, \mathcal{P} \leqslant n-1(R)\right)$ forms a complete cotorsion pair.

Proof. By Theorem 5.6 and Observation 5.7, we have $(1) \Leftrightarrow(3)$ and $(2) \Leftrightarrow(4)$.

$(1) \Leftrightarrow(2)$ Let $\operatorname{id}_{R} R \leqslant n-1$. By [6, Theorem 4.7] and the symmetric version of [21. Theorem], we have $\operatorname{id}_{R^{o p}} R \leqslant(n-1)+(n-2)=2 n-3$. Conversely, let $\operatorname{id}_{R^{o p}} R \leqslant n-1$. By [35, Theorem 7.5], we have $\operatorname{id}_{R} R \leqslant n-1$. Now the assertion follows from [40, Lemma A].

As a consequence of Corollary 5.8, we have the following

Corollary 5.9. For any artin algebra $R$, the following conditions are equivalent.

(1) $\operatorname{id}_{R} R \leqslant 1$.

(2) $\operatorname{id}_{R^{\text {op }}} R \leqslant 1$.

Furthermore, if $R$ is right quasi 1-Gorenstein, then they are equivalent to each of the following two statements.

(3) $\left(\mathcal{I}^{\leqslant 1}(R),{ }_{R} D(R)^{\perp_{2}}\right)$ forms a complete cotorsion pair.

(4) $\left({ }^{\perp_{2}} R, \mathcal{P} \leqslant 1(R)\right)$ forms a complete cotorsion pair.

Proof. The first assertion follows from [21, Corollary 2]. If $R$ is right quasi 1Gorenstein, then we get the second assertion by putting $n=2$ in Corollary 5.8 .

We use $\mathcal{I}(R)$ and $\mathcal{P}(R)$ to denote the subclasses of Mod $R$ consisting of injective and projective modules respectively. Putting $n=1$ in Corollary [5.8, we have the following

Corollary 5.10. For any artin algebra $R$, the following statements are equivalent.

(1) $R$ is self-injective.

(2) $\left(\mathcal{I}(R),{ }_{R} D(R)^{\perp_{1}}\right)$ forms a complete cotorsion pair (in this case, ${ }_{R} D(R)^{\perp_{1}}=$ $\left.\mathcal{I}(R)^{\perp_{1}}\right)$.

(3) $\left({ }^{\perp_{1}} R, \mathcal{P}(R)\right)$ forms a complete cotorsion pair (in this case, ${ }^{\perp_{1}} R={ }^{\perp_{1}} \mathcal{P}(R)$ ).

\section{Relative finitistic dimensions}

In this section, we introduce and study the finitistic $\mathcal{P}_{\omega}(R)$-injective dimension and the $\mathcal{I}_{\omega}(S)$-projective dimension of rings.

The finitistic $\mathcal{P}_{\omega}(R)$-injective dimension $\mathrm{F} \mathcal{P}_{\omega}$-id $R$ of $R$ is defined as

$$
\mathrm{FP}_{\omega} \text {-id } R:=\sup \left\{\mathcal{P}_{\omega}(R)-\operatorname{id}_{R} M \mid M \in \operatorname{Mod} R \text { and } \mathcal{P}_{\omega}(R)-\operatorname{id}_{R} M<\infty\right\} ;
$$


and the finitistic $\mathcal{I}_{\omega}(S)$-projective dimension $\mathrm{FI}_{\omega}$ - $\operatorname{pd} S$ of $S$ is defined as

$\mathrm{F} \mathcal{I}_{\omega}-\operatorname{pd} S:=\sup \left\{\mathcal{I}_{\omega}(S)-\operatorname{pd}_{S} N \mid N \in \operatorname{Mod} S\right.$ and $\left.\mathcal{I}_{\omega}(S)-\operatorname{pd}_{S} N<\infty\right\}$.

Lemma 6.1. For any $n \geqslant 0$ and $k \geqslant 1$, we have

(1) Let $\mathrm{T}$-cograde ${ }_{\omega} \operatorname{Ext}_{R}^{i+k}(\omega, M) \geqslant i$ for any $M \in \operatorname{Mod} R$ and $1 \leqslant i \leqslant n+1$. If $\mathrm{F} \mathcal{P}_{\omega}$-id $R=n$, then $\operatorname{pd}_{R} \omega \leqslant n+k$.

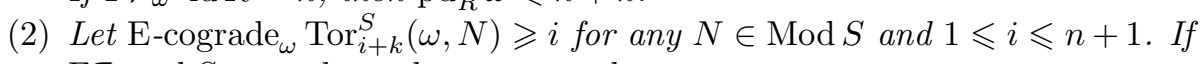
$\mathrm{FI}_{\omega}-\mathrm{pd} S=n$, then $\mathrm{pd}_{S^{o p}} \omega \leqslant n+k$.

Proof. (1) Let $M \in \operatorname{Mod} R$. By Proposition 5.4 there exists an exact sequence

$$
0 \rightarrow \operatorname{co} \Omega^{k-1}(M) \rightarrow X \rightarrow Y \rightarrow 0
$$

in $\operatorname{Mod} R$ with $X \in{ }_{R} \omega^{\perp_{n+2}}$ and $\mathcal{P}_{\omega}(R)-\operatorname{id}_{R} Y \leqslant n+1$. If $\mathrm{F} \mathcal{P}_{\omega}$-id $R=n$, then $\mathcal{P}_{\omega}(R)-\operatorname{id}_{R} Y \leqslant n$. Thus we have that

$$
\operatorname{Ext}_{R}^{n+k+1}(\omega, M) \cong \operatorname{Ext}_{R}^{n+2}\left(\omega, \operatorname{co}^{k-1}(M)\right) \cong \operatorname{Ext}_{R}^{n+1}(\omega, Y)=0
$$

and $\operatorname{pd}_{R} \omega \leqslant n+k$.

(2) Let $N \in \operatorname{Mod} S$. By Proposition 5.5, there exists an exact sequence

$$
0 \rightarrow Y^{\prime} \rightarrow X^{\prime} \rightarrow \Omega_{\mathcal{F}}^{k-1}(N) \rightarrow 0
$$

in $\operatorname{Mod} S$ with $X^{\prime} \in \omega_{S}{ }^{\top_{n+2}}$ and $\mathcal{P}_{\omega}(R)-\operatorname{id}_{S} Y^{\prime} \leqslant n+1$. If $\mathrm{F} \mathcal{I}_{\omega}$ - $\operatorname{pd} S=n$, then $\mathcal{I}_{\omega}(R)-\operatorname{pd}_{S} Y^{\prime} \leqslant n$. Thus we have that

$$
\operatorname{Tor}_{n+k+1}^{S}(\omega, N) \cong \operatorname{Tor}_{n+2}^{S}\left(\omega, \Omega_{\mathcal{F}}^{k-1}(N)\right) \cong \operatorname{Tor}_{n+1}^{S}\left(\omega, Y^{\prime}\right)=0
$$

and $\operatorname{pd}_{S^{o p}} \omega=\mathrm{fd}_{S^{o p}} \omega \leqslant n+k$.

Lemma 6.2. For any $n \geqslant 0$, we have

(1) Let $\mathrm{F} \mathcal{P}_{\omega}$-id $R \leqslant n$ and $N \in \operatorname{Mod} S$. If $\mathrm{T}$-cograde ${ }_{\omega} N \geqslant n+1$, then $N=0$.

(2) Let $\mathrm{F} \mathcal{I}_{\omega}-\operatorname{pd} S \leqslant n$ and $H \in \operatorname{Mod} R$. If E-cograde $\omega \geqslant n+1$, then $H=0$.

Proof. (1) Consider a projective resolution

$$
\cdots \rightarrow Q_{n+1} \rightarrow Q_{n} \rightarrow \cdots \rightarrow Q_{0} \rightarrow N \rightarrow 0
$$

of $N$ in $\operatorname{Mod} S$. If T-cograde $\omega_{\omega} N \geqslant n+1$, then we get an exact sequence

$$
0 \rightarrow M \rightarrow \omega \otimes_{S} Q_{n+1} \rightarrow \omega \otimes_{S} Q_{n} \rightarrow \cdots \rightarrow \omega \otimes_{S} Q_{1} \rightarrow \omega \otimes_{S} Q_{0} \rightarrow 0
$$

in $\operatorname{Mod} R$, where $M=\operatorname{Ker}\left(\omega \otimes_{S} Q_{n+1} \rightarrow \omega \otimes_{S} Q_{n}\right)$. By [34, Corollary 3.5], $Q \cong\left(\omega \otimes_{S} Q\right)_{*}$ canonically for any projective $Q$ in $\operatorname{Mod} S$, so $N \cong \operatorname{Ext}_{R}^{n+1}(\omega, M)$. Because $\mathrm{F} \mathcal{P}_{\omega}$-id $R \leqslant n$ by assumption, we have that $\mathcal{P}_{\omega}(R)-\operatorname{id}_{R} M \leqslant n$ and $N \cong$ $\operatorname{Ext}_{R}^{n+1}(\omega, M)=0$.

(2) Consider an injective resolution

$$
0 \rightarrow H \rightarrow I^{0} \rightarrow \cdots \rightarrow I^{n} \rightarrow I^{n+1} \rightarrow \cdots
$$

of $H$ in $\operatorname{Mod} R$. If E-cograde $\mathrm{e}_{\omega} H \geqslant n+1$, then we get an exact sequence

$$
0 \rightarrow I^{0}{ }_{*} \rightarrow \cdots \rightarrow I^{n}{ }_{*} \rightarrow I^{n+1}{ }_{*} \rightarrow N \rightarrow 0
$$

in $\operatorname{Mod} S$, where $N=\operatorname{Coker}\left(I^{n}{ }_{*} \rightarrow I^{n+1}{ }_{*}\right)$. By [32, Lemma 2.5(2)], $\omega \otimes_{S} I_{*} \cong I$ canonically for any injective $I$ in $\operatorname{Mod} R$, so $H \cong \operatorname{Tor}_{n+1}^{S}(\omega, N)$. Because $\mathrm{F} \mathcal{I}_{\omega}$ - pd $S \leqslant$ $n$ by assumption, we have that $\mathcal{I}_{\omega}\left(R^{o p}\right)-\operatorname{pd}_{S} N \leqslant n$ and $H \cong \operatorname{Tor}_{n+1}^{S}(\omega, N)=0$.

The following is the main result in this section.

Theorem 6.3. For any $k \geqslant 0$, we have 
(1) If $\mathrm{T}$-cograde $\operatorname{Ext}_{R}^{i+k}(\omega, M) \geqslant i$ for any $M \in \operatorname{Mod} R$ and $i \geqslant 1$, then $\mathrm{FP}_{\omega}-\mathrm{id} R \leqslant \mathrm{pd}_{R} \omega \leqslant \mathrm{FP}_{\omega}$-id $R+k$.

(2) If E-cograde $\operatorname{Tor}_{i+k}^{S}(\omega, N) \geqslant i$ for any $N \in \operatorname{Mod} S$ and $i \geqslant 1$, then $\mathrm{FI}_{\omega}-\mathrm{pd} S \leqslant \operatorname{pd}_{S^{o p}} \omega \leqslant \mathrm{F} \mathcal{I}_{\omega}-\operatorname{pd} S+k$.

Proof. (1) Let $\operatorname{pd}_{R} \omega=n(<\infty)$ and $M \in \operatorname{Mod} R$ with $\mathcal{P}_{\omega}(R)-\operatorname{id}_{R} M=m(<\infty)$. Then there exists an exact sequence

$$
0 \rightarrow M \stackrel{f^{0}}{\longrightarrow} \omega^{0} \stackrel{f^{1}}{\longrightarrow} \omega^{1} \rightarrow \cdots \stackrel{f^{m}}{\longrightarrow} \omega^{m} \rightarrow 0
$$

in $\operatorname{Mod} R$ with all $\omega^{i}$ in $\mathcal{P}_{\omega}(R)$. Since $\mathcal{P}_{\omega}(R) \subseteq \mathcal{B}_{\omega}(R)$ by [19, Corollary 6.1], we have $\mathcal{B}_{\omega}(R)-\operatorname{id}_{R} M \leqslant \mathcal{P}_{\omega}(R)-\operatorname{id}_{R} M<\infty$. If $m>n$, then it follows from [33, Theorem 4.2] that $\mathcal{B}_{\omega}(R)-\operatorname{id}_{R} M \leqslant n$ and $\operatorname{Im} f^{n} \in \mathcal{B}_{\omega}(R)$. On the other hand, we have the following exact and split sequence

$$
0 \rightarrow\left(\operatorname{Im} f^{n}\right)_{*} \rightarrow \omega^{n}{ }_{*} \rightarrow \cdots \rightarrow \omega^{m}{ }_{*} \rightarrow 0
$$

in $\operatorname{Mod} S$ with all $\omega^{i}{ }_{*}$ projective. So $\left(\operatorname{Im} f^{n}\right)_{*}$ is projective, and hence $\operatorname{Im} f^{n} \in$ $\mathcal{P}_{\omega}(R)$ by [19, Lemma 5.1(2)]. It yields that $\mathcal{P}_{\omega}(R)-\mathrm{id}_{R} M \leqslant n$, a contradiction. This proves $\mathrm{FP}_{\omega}$ - id $R \leqslant \operatorname{pd}_{R} \omega$.

In the following, we will prove $\operatorname{pd}_{R} \omega \leqslant \mathrm{FP}_{\omega}$-id $R+k$. The case for $k \geqslant 1$ follows from Lemma 6.1(1). Now suppose that $k=0$ and $\mathrm{FP}_{\omega}$-id $R=n(<\infty)$. Let $M \in \operatorname{Mod} R$. Then T-cograde $\operatorname{Ext}_{R}^{n+1}(\omega, M) \geqslant n+1$ by assumption. It follows from Lemma $6.2(1)$ that $\operatorname{Ext}_{R}^{n+1}(\omega, M)=0$ and $\operatorname{pd}_{R} \omega \leqslant n$.

(2) Let $\operatorname{pd}_{S^{o p}} \omega=n(<\infty)$ and $N \in \operatorname{Mod} S$ with $\mathcal{I}_{\omega}(S)-\operatorname{pd}_{S} N=m(<\infty)$. Then there exists an exact sequence

$$
0 \rightarrow U_{m} \stackrel{g_{m}}{\longrightarrow} \cdots \rightarrow U_{1} \stackrel{g_{1}}{\longrightarrow} U_{0} \stackrel{g_{0}}{\longrightarrow} N \rightarrow 0
$$

in $\operatorname{Mod} S$ with all $U_{i}$ in $\mathcal{I}_{\omega}(S)$. Since $\mathcal{I}_{\omega}(S) \subseteq \mathcal{A}_{\omega}(S)$ by [19, Corollary 6.1], we have $\mathcal{A}_{\omega}(S)-\operatorname{pd}_{S} N<\infty$. If $m>n$, then it follows from the dual result of [33, Theorem 4.2] that $\mathcal{A}_{\omega}(S)-\operatorname{pd}_{S} N \leqslant n$ and $\operatorname{Im} g_{n} \in \mathcal{A}_{\omega}(S)$. On the other hand, we have the following exact and split sequence

$$
0 \rightarrow \omega \otimes_{S} U_{m} \rightarrow \cdots \rightarrow \omega \otimes_{S} U_{n} \rightarrow \omega \otimes_{S} \operatorname{Im} g_{n} \rightarrow 0
$$

in $\operatorname{Mod} R$ with all $\omega \otimes_{S} U_{i}$ injective. So $\omega \otimes_{S} \operatorname{Im} g_{n}$ is injective, and hence $\operatorname{Im} g_{n} \in$ $\mathcal{I}_{\omega}(S)$ by [19, Lemma 5.1(3)]. It yields that $\mathcal{I}_{\omega}(S)-\operatorname{pd}_{S} N \leqslant n$, a contradiction. This proves $\mathrm{F} \mathcal{I}_{\omega}-\mathrm{pd} S \leqslant \operatorname{pd}_{S^{o p}} \omega$.

In the following, we will prove $\operatorname{pd}_{S^{o p}} \omega \leqslant \mathrm{FI}_{\mathcal{I}}$ - $\mathrm{pd} S+k$. The case for $k \geqslant 1$ follows from Lemma 6.1(2). Now suppose that $k=0$ and $\mathrm{FI}_{\omega^{-}} \operatorname{pd} S=n$. Let $N \in \operatorname{Mod} S$. Then E-cograde $\operatorname{Tor}_{n+1}^{S}(\omega, N) \geqslant n+1$ by assumption. It follows from Lemma $6.2(2)$ that $\operatorname{Tor}_{n+1}^{S}(\omega, N)=0$ and $\operatorname{pd}_{S^{o p}} \omega=\operatorname{fd}_{S^{o p}} \omega \leqslant n$.

Putting $k=0$ in Theorem 6.3 we immediately get the following

\section{Corollary 6.4.}

(1) If $\mathrm{T}$-cograde ${ }_{\omega} \operatorname{Ext}_{R}^{i}(\omega, M) \geqslant i$ for any $M \in \operatorname{Mod} R$ and $i \geqslant 1$, then $\mathrm{FP}_{\omega}$-id $R=\operatorname{pd}_{R} \omega$

(2) If $\mathrm{E}$-cograde $\omega \operatorname{Tor}_{i}^{S}(\omega, N) \geqslant i$ for any $N \in \operatorname{Mod} S$ and $i \geqslant 1$, then $\mathrm{F} \mathcal{I}_{\omega}$-pd $S$ $=\operatorname{pd}_{S^{o p}} \omega$.

The following is an immediate consequence of Corollaries 4.2 and 6.4 
Corollary 6.5. If $\omega$ satisfies the $n$-cograde condition for all $n$, then

$$
\mathrm{FP}_{\omega}-\mathrm{id} R=\operatorname{pd}_{R} \omega \text { and } \mathrm{FI}_{\omega}-\mathrm{pd} S=\mathrm{pd}_{S^{o p}} \omega .
$$

Combining Theorem 4.19 with the case for $k=1$ in Theorem 6.3, we get the following

Corollary 6.6. We have

$$
\begin{gathered}
\mathrm{FP}_{\omega}-\mathrm{id} R \leqslant \operatorname{pd}_{R} \omega \leqslant \mathrm{F} \mathcal{P}_{\omega}-\mathrm{id} R+1 \text { and } \\
\mathrm{FI}_{\omega}-\operatorname{pd} S \leqslant \operatorname{pd}_{S^{o p}} \omega \leqslant \mathrm{F} \mathcal{I}_{\omega}-\operatorname{pd} S+1,
\end{gathered}
$$

if either of the following conditions is satisfied.

(1) $\mathrm{T}_{\text {-cograde }} \operatorname{Ext}_{R}^{i+1}(\omega, M) \geqslant i$ for any $M \in \operatorname{Mod} R$ and $i \geqslant 1$.

(2) E-cograde $_{\omega} \operatorname{Tor}_{i+1}^{S}(\omega, N) \geqslant i$ for any $N \in \operatorname{Mod} S$ and $i \geqslant 1$.

Corollary 6.7. If $\omega$ satisfies the right quasi $n$-cograde condition for all $n$, then

$$
\mathrm{FP}_{\omega} \text {-id } R=\operatorname{pd}_{R} \omega \text { and } \mathrm{FI}_{\omega}-\mathrm{pd} S \leqslant \mathrm{pd}_{S^{o p}} \omega \leqslant \mathrm{F} \mathcal{I}_{\omega}-\operatorname{pd} S+1 .
$$

Proof. The former equality follows from Proposition4.12 and Corollary 6.4 (1), and the later inequalities follow from the definition of the right quasi $n$-cograde condition and Corollary 6.6.

Observation 6.8. Let $R$ be an artin algebra and $R_{R} \omega_{S}={ }_{R} D(R)_{R}$. Then we have

(1) By Observation 5.7, we have

$$
\begin{gathered}
\mathrm{F} \mathcal{P}_{\omega}-\mathrm{id} R=\mathrm{FID} R:=\sup \left\{\operatorname{id}_{R} M \mid M \in \operatorname{Mod} R \text { and } \operatorname{id}_{R} M<\infty\right\}, \\
\mathrm{F} \mathcal{I}_{\omega^{-}} \text {pd } S=\text { FPD } R:=\sup \left\{\operatorname{pd}_{R} N \mid N \in \operatorname{Mod} R \text { and } \operatorname{pd}_{R} N<\infty\right\} .
\end{gathered}
$$

(2) If $R$ is right (or left) quasi $n$-Gorenstein for all $n$, then $\operatorname{id}_{R^{o p}} R=\operatorname{id}_{R} R$ ([21, Corollary 4]).

As a consequence of the above results, we have the following

Corollary 6.9. Let $R$ be an artin algebra. Then we have

(1) If $R$ satisfies the Auslander condition (that is, $R$ is Auslander $n$-Gorenstein for all $n$ ), then

$$
\text { FPD } R^{o p}=\text { FID } R^{o p}=\operatorname{id}_{R^{o p}} R=\operatorname{id}_{R} R=\operatorname{FPD} R=\text { FID } R .
$$

(2) If $R$ satisfies the right quasi Auslander condition (that is, $R$ is right quasi $n$-Gorenstein for all $n$ ), then

$$
\text { FPD } R \leqslant \operatorname{FID} R=\operatorname{id}_{R^{o p}} R=\operatorname{id}_{R} R \leqslant \operatorname{FPD} R+1 .
$$

Proof. In view of Example 4.20, Observations 5.7 and 6.8, the assertions follow from Corollaries 6.5 and 6.7 respectively.

Acknowledgements. This research was partially supported by NSFC (Grant Nos. 11571164, 11501144), a Project Funded by the Priority Academic Program Development of Jiangsu Higher Education Institutions and NSF of Guangxi Province of China (Grant No. 2016GXNSFAA380151). The authors thank the referee for the useful suggestions. 


\section{REFERENCES}

[1] F. W. Anderson and K. R. Fuller, Rings and Categories of Modules, Graduate Texts in Mathematics, Vol. 13, Springer-Verlag, New York, 1992.

[2] T. Araya, R. Takahashi and Y. Yoshino, Homological invariants associated to semidualizing bimodules, J. Math. Kyoto Univ. 45 (2005), 287-306.

[3] I. Assem, D. Simson and A. Skowroński, Elements of the Representation Theory of Associative Algebras, Vol. 1, Techniques of Representation Theory, London Math. Soc. Stud. Texts 65, Cambridge Univ. Press, Cambridge, 2006.

[4] M. Auslander and M. Bridger, Stable Module Theory, Memoirs Amer. Math. Soc. 94, Amer. Math. Soc., Providence, RI, 1969.

[5] M. Auslander and I. Reiten, $k$-Gorenstein algebras and syzygy modules, J. Pure Appl. Algebra 92 (1994), 1-27.

[6] M. Auslander and I. Reiten, Syzygy modules for noetherian rings, J. Algebra 183 (1996), $167-185$.

[7] M. Auslander, I. Reiten and S. O. Smalø, Representation Theory of Artin Algebras, Corrected reprint of the 1995 original, Cambridge Stud. in Adv. Math. 36, Cambridge Univ. Press, 1997.

[8] A. Beligiannis and I. Reiten, Homological and Homotopical Aspects of Torsion Theories, Mem. Amer. Math. Soc. 188(883), Amer. Math. Soc., Providence, RI, 2007.

[9] L. Bican, R. El Bashir and E. E. Enochs, All modules have flat covers, Bull. London Math. Soc. 33 (2001), 385-390.

[10] J. E. Björk, The Auslander condition on Noetherian rings, in: Séminaire d'Algèbre Paul Dubreil et Marie-Paul Malliavin, 39ème Année, Paris, 1987/1988. Lect. Notes in Math. 1404, Springer- Verlag, Berlin, 1989, pp.137-173.

[11] H. Cartan and S. Eilenberg, Homological Algebra, with an appendix by D. A. Buchsbaum, reprint of the 1956 original, Princeton Landmarks in Math., Princeton Univ. Press, Princeton, 1999.

[12] J. Coates, P. Schneider and R. Sujatha, Modules over Iwasawa algebras, Institute J. Math. Jussieu 2 (2003), 73-108.

[13] E. E. Enochs, Injective and flat covers, envelopes and resolvents, Israel J. Math. 39 (1981), 189-209.

[14] E. E. Enochs and O. M. G. Jenda, Relative Homological Algebra. de Gruyter Exp. in Math. 30, Walter de Gruyter, Berlin, New York, 2000.

[15] K. Erdmann, T. Holm, O. Iyama and J. Schröer, Radical embeddings and representation dimension, Adv. Math. 185 (2004), 159-177.

[16] R. M. Fossum, P. A. Griffith and I. Reiten, Trivial Extensions of Abelian Categories. Homological Algebra of Trivial Extensions of Abelian Categories with Applications to Ring Theory, Lecture Notes in Math. 456, Springer-Verlag, Berlin, 1975.

[17] R. Göbel and J. Trlifaj, Approximations and Endomorphism Algebras of Modules, de Gruyter Exp. in Math. 41, Walter de Gruyter, Berlin, New York, 2006.

[18] P. A. Guil Asensio and I. Herzog, Left cotorsion rings, Bull. London Math. Soc. 36 (2004), 303-309.

[19] H. Holm and D. White, Foxby equivalence over associative rings, J. Math. Kyoto Univ. 47 (2007), 781-808.

[20] Z. Y. Huang, Extension closure of k-torsionfree modules, Comm. Algebra 27 (1999), 1457-1464.

[21] Z. Y. Huang, Selforthogonal modules with finite injective dimension II, J. Algebra 264 (2003), 262-268.

[22] Z. Y. Huang, Syzygy modules for quasi k-Gorenstein rings, J. Algebra 299 (2006), 21-32.

[23] Z. Y. Huang, Proper resolutions and Gorenstein categories, J. Algebra 393 (2013), 142169.

[24] Z. Y. Huang and O. Iyama, Auslander-type conditions and cotorsion pairs, J. Algebra 318 (2007), 93-110.

[25] Z. Y. Huang and H. R. Qin, Homological behavior of Auslander's k-Gorenstein rings, Algebr. Represent. Theory 15 (2012), 835-853.

[26] Y. Iwanaga and H. Sato, On Auslander's n-Gorenstein rings, J. Pure Appl. Algebra 106 (1996), 61-76. 
[27] O. Iyama, Auslander correspondence, Adv. Math. 210 (2007), 51-82.

[28] O. Iyama, Cluster tilting for higher Auslander algebras, Adv. Math. 226 (2011), 1-61.

[29] F. Mantese and I. Reiten, Wakamatsu tilting modules, J. Algebra 278 (2004), 532-552.

[30] W. K. Nicholson, Semiregular modules and rings, Canad. J. Math. 28 (1976), 1105-1120.

[31] S. Sather-Wagstaff, Semidualizing Modules, Mathematics Monograph, Preprint, avail-

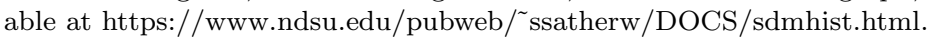

[32] X. Tang and Z. Y. Huang, Homological aspects of the dual Auslander transpose, Forum Math. 27 (2015), 3717-3743.

[33] X. Tang and Z. Y. Huang, Homological aspects of the dual Auslander transpose II, Kyoto J. Math. 57 (2017), 17-53.

[34] X. Tang and Z. Y. Huang, Homological aspects of the adjoint cotranspose, Colloq. Math. 150 (2017), 293-311.

[35] X. Tang and Z. Y. Huang, Coreflexive modules and semidualizing modules with finite projective dimension, Taiwanese J. Math. 21 (2017), 1283-1324.

[36] X. Tang and Z. Y. Huang, Two filtration results for modules with applications to the Auslander condition, Colloq. Math. (to appear), Preprint is available at http://math.nju.edu.cn/ ${ }^{\sim}$ huangzy/

[37] T. Wakamatsu, On modules with trivial self-extensions, J. Algebra 114 (1988), 106-114.

[38] T. Wakamatsu, Stable equivalence for self-injective algebras and a generalization of tilting modules, J. Algebra 134 (1990), 298-325.

[39] T. Wakamatsu, Tilting modules and Auslander's Gorenstein property, J. Algebra 275 (2004), 3-39.

[40] A. Zaks, Injective dimension of semiprimary rings, J. Algebra 13 (1969), 73-89.

College of Science, Guilin University of Technology, Guilin 541004, Guangxi Province, P.R. CHINA,

E-mail address: tx5259@sina.com.cn

Department of Mathematics, Nanjing University, Nanjing 210093, Jiangsu Province, P.R. ChINA

E-mail address: huangzy@nju.edu.cn

$U R L:$ http://math.nju.edu.cn/ ${ }^{\text {huangzy/ }}$ 